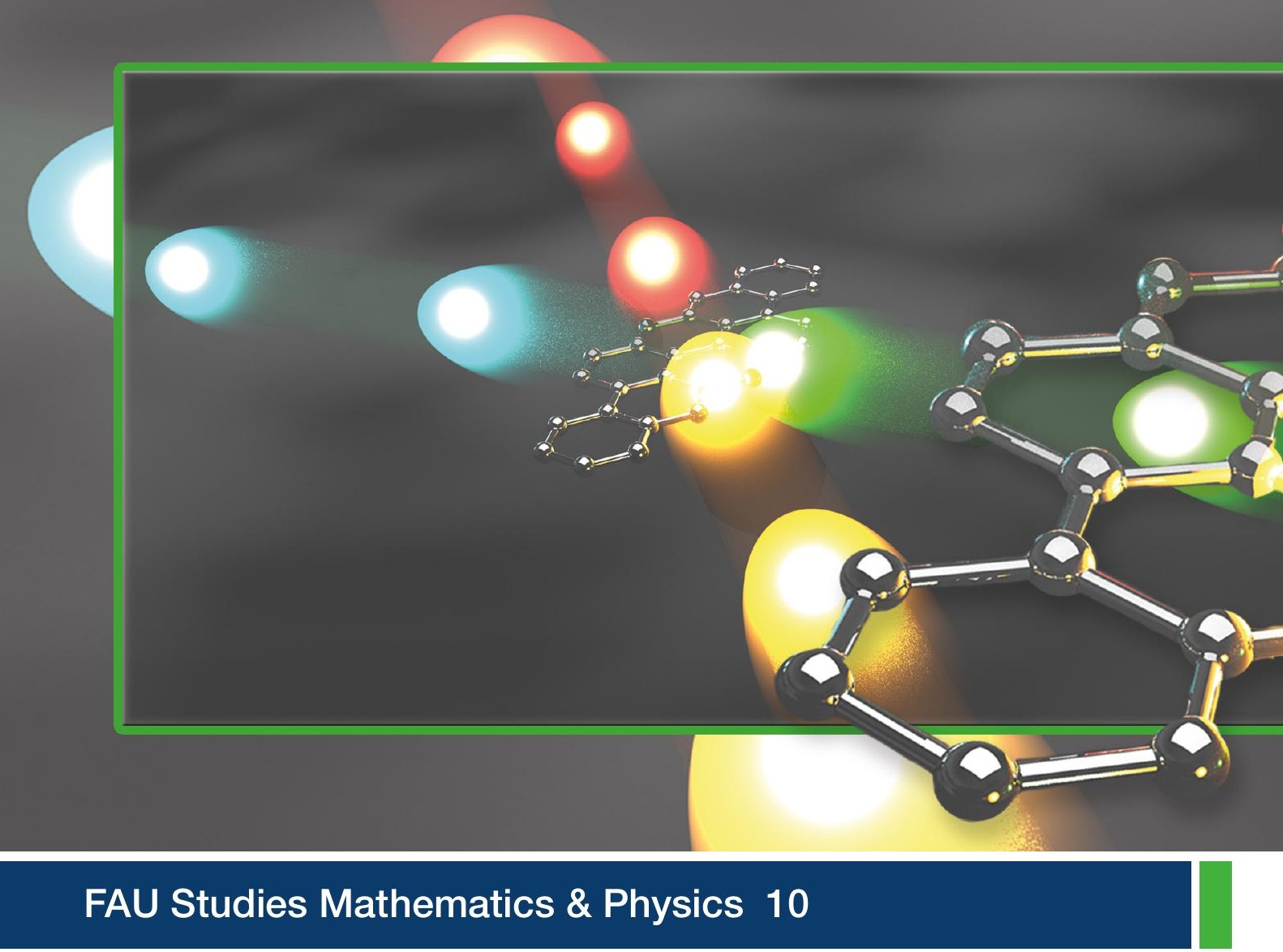

Andreas Johannes Maser

Few-photon coherent nonlinear optics with a single molecule 

Andreas Maser

Few-photon coherent nonlinear optics with a single molecule 


\section{FAU Studies Mathematics \& Physics}

\section{Band 10}

Herausgeber der Reihe:

Prof. Dr. Karl-Hermann Neeb und Prof. Dr. Klaus Mecke 
Andreas Maser

Few-photon coherent nonlinear optics with a single molecule

Erlangen

FAU University Press

2017 
Bibliografische Information der Deutschen Nationalbibliothek:

Die Deutsche Nationalbibliothek verzeichnet diese Publikation in der Deutschen Nationalbibliografie; detaillierte bibliografische Daten sind im Internet über http://dnb.d-nb.de abrufbar.

Das Werk, einschließlich seiner Teile, ist urheberrechtlich geschützt. Die Rechte an allen Inhalten liegen bei ihren jeweiligen Autoren. Sie sind nutzbar unter der Creative Commons Lizenz BY-NC-ND.

Der vollständige Inhalt des Buchs ist als PDF über den OPUS Server der Friedrich-Alexander-Universität Erlangen-Nürnberg abrufbar: https://opus4.kobv.de/opus4-fau/home

Verlag und Auslieferung:

FAU University Press, Universitätsstraße 4, 91054 Erlangen

Druck: docupoint $\mathrm{GmbH}$

ISBN: 978-3-96147-023-5 (Druckausgabe) eISBN: 978-3-96147-024-2 (Online-Ausgabe) ISSN: $2196-7482$ 


\title{
Few-photon coherent nonlinear optics with a single molecule
}

Der Naturwissenschaftlichen Fakultät der Friedrich-Alexander-Universität Erlangen-Nürnberg zur

Erlangung des Doktorgrades Dr. rer. nat.

\author{
vorgelegt von \\ Andreas Johannes Maser \\ aus Nürnberg
}


Als Dissertation genehmigt von der Naturwissenschaftlichen Fakultät

der Friedrich-Alexander-Universität Erlangen-Nürnberg

Tag der mündlichen Prüfung: 09.11.2016

Vorsitzender des Promotionsorgans: Prof. Dr. Georg Kreimer

Gutachter: Prof. Dr. Vahid Sandoghdar

Prof. Dr. Brahim Lounis 


\section{Summary}

This thesis covers the interaction of light with single dibenzanthanthrene (DBATT) dye molecules. DBATT serves as a model for a quantum-mechanical two-level system. By means of strong focusing of the incident light and cooling of the dye molecules to temperatures below $2 \mathrm{~K}$, a particularly efficient light-matter interaction can be realized. This enables the observation of the nonlinearity inherent to a two-level system, e.g., in the form of saturation of the fluorescence signal, already with light beams containing only a few photons per lifetime of the excited molecular state.

If two light beams with different frequencies are sent to a single molecule, various nonlinear phenomena occur. These processes can be exploited to coherently manipulate the transmission of a beam focused onto a single molecule with a second light beam. The occurrent effects, i.e., the AC-Stark shift, stimulated Rayleigh scattering, and three-photon amplification, are detected in the transmission signal. In addition, four-wave mixing and the dependence of the excited state population on the phase difference of the two incident beams are demonstrated by the use of measurements with subnanosecond time resolution. These results show the possible application of organic dye molecules in the field of quantum information processing, where nonlinearities on the single photon and single emitter level are sought-after.

Within this work, the experimental and theoretical principles of single molecule spectroscopy are discussed. Particular attention is turned to the investigation of the coherent light-matter interaction using transmission measurements. A significant change of this signal via the scattering of a single molecule requires a strong light-matter coupling. To quantify the efficiency of this interaction, the maximum possible coupling of a focused beam and a single emitter is discussed. The data shows that the achieved coupling is typically $5 \%$ of the theoretical maximum.

Subsequently, the interaction of a molecule with two light fields with different frequencies is investigated. The appearing nonlinear effects are described qualitatively within the dressed-atom model and quantitatively with a Fourier ansatz. The experimental techniques are explained in detail and the measurement results are presented and discussed. Moreover, a possible application of a single molecule as a few-photon optical switch is outlined. 
In the end, an outlook is given on interesting future investigations including nonlinear optics with single-photon pulses and with dipole-coupled molecules. 


\section{Zusammenfassung}

Die vorliegende Dissertation behandelt die Wechselwirkung von Licht mit einzelnen Dibenzanthanthren (DBATT) Farbstoffmolekülen. DBATT dient hierbei als Modell für ein quantenmechanisches Zweiniveau-System. Durch starke Fokussierung des einfallenden Lichts und Kühlung der Farbstoffmoleküle auf eine Temperatur unter $2 \mathrm{~K}$ kann eine besonders effiziente LichtMaterie-Wechselwirkung realisiert werden. Diese ermöglicht es bereits mit einem Lichtstrahl, der nur wenige Photonen pro Lebenszeit des angeregten Molekülzustandes enthält, die inhärente Nichtlinearität eines ZweiniveauSystems, zum Beispiel in Form von Sättigung des Fluoreszenzsignals, zu beobachten.

Verwendet man zwei Lichtstrahlen mit unterschiedlichen Frequenzen, so ergeben sich verschiedene nichtlineare Phänomene. Diese Prozesse können dazu genutzt werden, die Transmission eines Strahls, der auf ein einzelnes Molekül fokussiert ist, mithilfe eines zweiten Lichtstrahls kohärent zu beeinflussen. Die hierbei auftretenden Effekte, die AC-Starkverschiebung, stimulierte Rayleigh-Streuung und drei-Photonenverstärkung werden im Transmissionssignal nachgewiesen. Durch Messungen mit subnanosekunden Zeitauflösung wird außerdem Vier-Wellenmischung und die Abhängigkeit der Population im angeregten Zustand von der relativen Phase der beiden einfallenden Lichtstrahlen demonstriert. Diese Ergebnisse zeigen die Einsatzmöglichkeit organischer Farbstoffmoleküle im Bereich der Quanteninformationsverarbeitung, wo Nichtlinearitäten auf dem Niveau einzelner Photonen und einzelner Emitter benötigt werden.

In dieser Arbeit werden die experimentellen und theoretischen Grundlagen der Einzelmolekülspektroskopie besprochen. Ein besonderes Augenmerk liegt hierbei auf der Untersuchung der kohärenten Licht-Materie-Wechselwirkung mit Hilfe von Transmissionsmessungen. Eine signifikante Änderung dieses Signals durch die Streuung eines einzelnen Moleküls erfordert eine starke Licht-Materie-Wechselwirkung. Um die Effizienz dieser Interaktion zu quantifizieren, wird die maximal erreichbare Kopplung eines fokussierten Lichtstrahls an einen einzelnen Emitter erörtert. Dabei zeigt sich, dass die Kopplung im verwendeten System typischerweise bei $5 \%$ des theoretischen Maximums liegt. 
Anschließend wird die Wechselwirkung eines Moleküls mit zwei Lichtfeldern unterschiedlicher Frequenz untersucht. Die sich dabei ergebenden nichtlineare Effekte werden im Rahmen des dressed atom-Modells qualitativ und mittels eines Fourieransatzes quantitativ beschrieben. Die für den Nachweis notwendigen experimentellen Techniken werden ausführlich dargelegt und die Ergebnisse der Messungen präsentiert und diskutiert. Außerdem wird eine Möglichkeit aufgezeigt einzelne Moleküle als mit wenigen Photonen aktivierbare, optische Schalter zu verwenden.

Zum Schluss wird ein Ausblick auf interessante, zukünftige Untersuchungen gegeben, wie zum Beispiel nichtlineare Optik mit EinzelphotonenPulsen oder mit dipolgekoppelten Molekülen. 


\section{Contents}

1 Introduction 1

2 Single molecule optics 7

2.1 Basic concepts of single molecule spectroscopy . . . . . . . . 9

2.1.1 Photo-physical properties of dye molecules in organic matrices ......................... 9

2.1.2 Addressing a single molecule . . . . . . . . . . 12

2.1.3 DBATT in naphthalene ............ 13

2.1.4 Interaction of light with a single molecule . . . . . . 14

2.1.5 Fluorescence ................ 19

2.1.6 Extinction ............... 21

2.2 Experimental implementation ............ 26

2.2.1 Optical setup ............... 26

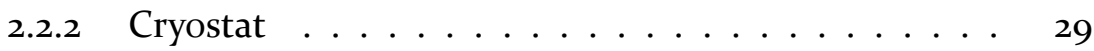

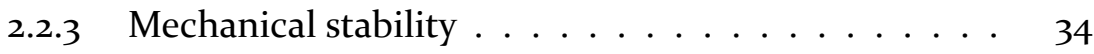

2.2.4 Sample preparation ............ 35

2.3 Single molecule characterization measurements . . . . . 36

2.3.1 Wide-field illumination ............ 36

2.3.2 Spectral characterization ........... 41

2.3.3 Spatial characterization ........... 41

2.4 Molecule pairs ................. 45

3 Photon-molecule coupling efficiency 49

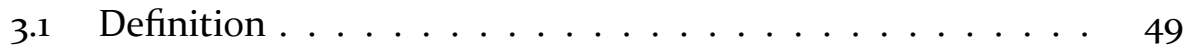

3.2 Measurement................ 53

3.3 Improving the coupling efficiency ........... 58

3.3.1 Gallium phosphide SIL ........... 60

3.3.2 Spherical mirror ............. 64

4 Nonlinear optics with a single molecule $\quad 69$

4.1 Probing a dressed two-level system . . . . . . . . . . . 70

4.2 Two beams interacting with a TLS .......... 75

4.2.1 Fluorescence .............. 78

4.2.2 Transmission ............... 82 
4.3 Transmission measurements . . . . . . . . . . . . . 89

4.3.1 Modifications to the setup ........... . 89

4.3.2 Measurement and evaluation sequence . . . . . . . 93

4.3 .3 Experimental results . . . . . . . . . . . . . 96

4.4 Few-photon optical switching . . . . . . . . . 100

4.5 Time-dependent measurements . . . . . . . . . . . 103

4.5.1 Setup modifications and measurement technique . . 103

4.5 .2 Experimental results . . . . . . . . . . . 107

5 Outlook 113 


\section{Introduction}

Under most circumstances light does not interact with light. Thus, if one crosses the beams of two flash lights, they do not affect each other. While this property is a desirable feature for certain applications, e.g., optical data transmission, there are also fields where one explicitly requires light-light interaction. A prominent example is light-based information processing. To perform computation with optical bits, it is necessary that one bit can affect the state of another one. A way of realizing such gate operations would be switching light by light, just as electric currents are used to switch other electric currents in common electronic devices. The required interaction can be achieved via nonlinear optics.

"Nonlinear optics is the study of phenomena that occur as a consequence of the modification of the optical properties of a material system by the presence of light" [1]. One of the first experimental demonstrations of such a nonlinear effect was the observation of saturation in the phosphorescence of fluorescein in 1941 [2]. As shown in Fig. 1.1, the intensity of the emitted light was not increasing proportional to the intensity of the excitation light, as would be expected if the optical properties were independent of the presence of light. Instead, the absorption decreased, and the power of the emitted light tended towards a maximum value.

Under normal conditions most materials show a linear optical behavior. Therefore, the observation of nonlinear effects typically requires high intensities only achievable with laser beams. In this regime, a plethora of phenomena can be explored, which are caused by the nonlinear material response: The energy of several photons can be combined to create a new wave at the sum frequency [3]. The opposite process is also possible: The energy of one photon can be split into two or more photons of lower frequency, which is known as spontaneous parametric down conversion [4, 5]. Apart from the generation of new frequencies, intense laser beams can change the refractive index of a material. This optical Kerr effect causes phenomena like selffocusing [6] and self-phase modulation [7-9].

These and other nonlinear effects find widespread applications in science and industry. Sum and difference frequency generation is used to generate 


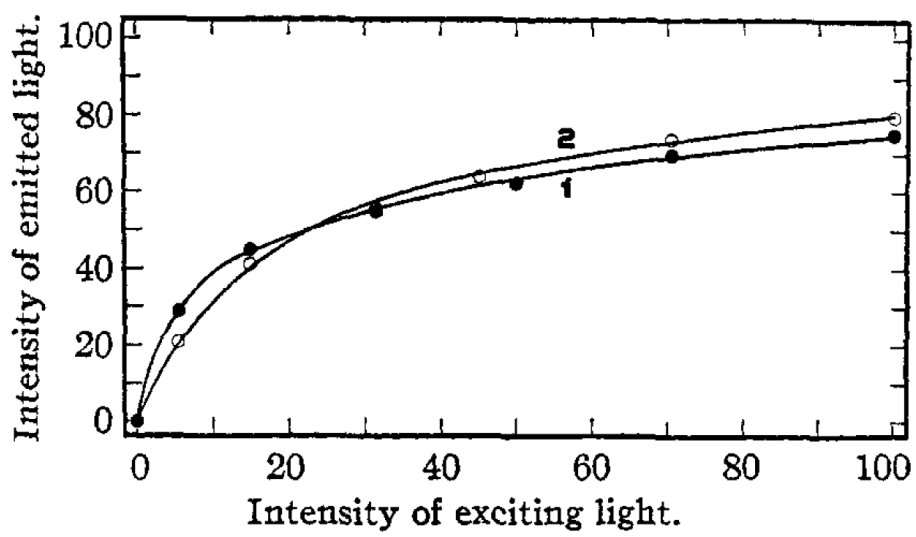

Fig. 5.-Saturation of phosphorescence of fluorescein in boric acid: (1) $+18^{\circ} ;(2)-185^{\circ}$.

Figure 1.1: Observation of saturation in the phosphorescence signal of fluorescein in boric acid. Reprinted with permission from [2]. Copyright 1941 American Chemical Society.

laser light at wavelengths that are not directly accessible, e.g., most commercial green laser pointers employ a crystal offering a suitable nonlinearity to convert infrared light at $1064 \mathrm{~nm}$ into visible radiation at $532 \mathrm{~nm}$. Spontaneous parametric down conversion can be used as a source for entangled photon pairs $[5,10,11]$ and heralded single photons [12] and is thus heavily utilized in quantum information and quantum communication [13]. The optical Kerr effect is used for the generation of ultra-short laser pulses via mode-locking [14].

Indeed the laser itself is an example of nonlinear optics: The pump beam changes the optical properties of the gain medium such that another beam passing through it is no longer attenuated but instead amplified. Of course, there are many more nonlinear phenomena and applications which can be found in standard textbooks $[1,15,16]$.

Even though the small cross section of nonlinear processes $[1,16]$ needs to be compensated in most experiments with high intensity light fields and macroscopic samples, there has been some progress in studying nonlinear optics with nanoscopic samples. For example, second harmonic generation and two-photon excitation have been observed using organic nano-crystals $[17]$ or metallic nano-structures $[18,19]$.

Albeit being on the nano-scale, these samples still contain several hundred thousand atoms. On the level of single emitters, optical nonlinearities were 
demonstrated with single organic dye molecules. Amongst other effects, researchers reported the observation of two-photon excitation [20, 21], Rabi oscillations [22, 23], the AC-Stark shift [24] and the hyper-Raman transition [25].

While in the aforementioned experiments the sample was reduced to the most fundamental unit, the light intensities were still far away from the single photon level. To enter the field of quantum nonlinear optics [26], where single photons already cause a significant nonlinear response, the light-matter coupling efficiency has to be further increased. Besides the fundamental interest in studying nonlinear optics at the single-emitter single-photon limit, this regime offers promising perspectives for classical and quantum information processing. An optical transistor would be more energy efficient than its electric counterpart if the device could be switched with less than $10 \mathrm{aJ}$, corresponding to 30 photons at a wavelength of $600 \mathrm{~nm}[26,27]$. At the single photon level, such a transistor could be used to implement conditional gate operations between two photons in quantum networks [28], which are required for a universal quantum computer [29]. If the optical transistor offers a sufficiently high gain [30, 31], i.e., a single control photon can switch a beam containing many photons, the generation of macroscopic, nonclassical states of light, e.g., Schrödinger cat states, becomes feasible [32]. Furthermore, the transistor would enable highly efficient single photon detection, which could be even nondestructive if the control photon can be retrieved after switching [33].

Placing a single emitter inside a high finesse cavity is one way of realizing a sufficiently strong light matter interaction to enter the the regime of quantum nonlinear optics. The cavity forces each photon to pass the emitter several hundred thousand times, thus compensating the low single pass interaction probability. Such a single emitter-cavity system can be realized in a variety of ways. The "classical" approach consists of a single atom which is trapped or falling through the space between two high reflectivity mirrors $[34,35]$. The cavity can be also a whispering gallery mode resonator, where light is circulating inside a disc or sphere guided by total internal reflection $[36,37]$. An atom in the vicinity of such a structure can interact strongly with the light in the resonator [38]. If an artificial atom, like a quantum dot, is used instead of a real atom, the emitter can be directly integrated into the cavity by lithographically fabricating both inside the same substrate [39-41].

Taking advantage of the strong light-emitter coupling, several remarkable nonlinear effects at the single-photon single-emitter scale were observed: the 
phase shift of light transmitted through [34] or reflected off a cavity containing a single emitter [40] was shown to change significantly if none or one single photon is present inside the cavity. A more recent experiment demonstrated a variation of the phase shift from zero to $\pi$ depending on whether one or two photons impinge on the cavity [38]. In a different work, a single photon could control if a second beam is reflected or transmitted through a cavity [41]. Besides controlling transmission and phase shift, a single emitter coupled to a cavity was also used to manipulate the photon statistics of light, thus turning a coherent input state into a nonclassical state of light [39].

The experiments presented in this thesis adopt a more straight forward approach towards efficient light-matter interaction. We focus the incident light as strongly as possible onto a single dibenzanthanthrene (DBATT) molecule embedded in a naphthalene crystal at liquid helium temperatures. Under these conditions, DBATT behaves very similar to an ideal two-level system resulting in a large scattering cross section. The combination of tight focusing with a near perfect two-level system leads to a high interaction probability of light and emitter without the need for an optical cavity. Thus, we can observe the onset of a nonlinear response, namely saturation, already for less than one incident photon per excited state lifetime.

Making use of this large coupling efficiency, we present direct measurements of the coherent, nonlinear interaction of a single molecule with two laser beams [42]. A strong pump beam with fixed frequency is used to modify the optical properties of a single molecule. We then detect the altered response by measuring the transmitted power of a weak probe beam, which is scanned across the resonance of the molecule. In this way, the single molecule effectively mediates an interaction of pump and probe. We observe all features predicted in the so-called Mollow absorption spectrum [43]: The AC-Stark shift, stimulated Rayleigh scattering and three-photon or hyperRaman amplification. These effects can be in principle demonstrated with only 30 pump photons per lifetime, with the fundamental limit being at 1.5 photons per lifetime. Hence, the "strong" pump beam is only strong in the sense that it efficiently drives the molecule, but by no means strong in absolute power. Exploiting this nonlinearity, we furthermore demonstrate the feasibility of optically switching a light beam with just a few pump photons [42].

This thesis is organized as follows. In chapter 2 , we introduce the system, DBATT molecules embedded in a naphthalene crystal and the optical setup. Furthermore, the semi-classical description of the interaction of a two-level system with a monochromatic electromagnetic field is reviewed 
and used to model fluorescence and transmission signals. In the last part, we discuss standard characterization measurements and experiments with molecule pairs.

Chapter 3 addresses the topic of light-matter coupling efficiency. We begin by deriving the maximum possible response of a two-level system to an external driving field and then compare this to the experimentally achieved values. In the end, we consider possible ways to increase the coupling efficiency and also present first experimental results.

In chapter 4, we discuss the nonlinear interaction of a single molecule with two laser beams. At first, a qualitative explanation of the expected effects is given using the dressed atom picture. To obtain a quantitative description, we then extend the optical Bloch equations, which were recapitulated in chapter 2, to the case of a bichromatic excitation field. In the third and fourth section of this chapter, we introduce the required modifications to the optical setup, present the experimental results of the transmission measurements and elaborate on possible applications in the field of few-photon switching. We end this chapter with discussing the measurements of the time-dependent transmission and fluorescence signals.

Finally, the last chapter gives an outlook on possible future experiments extending the investigation of nonlinear optics to more complex system, like dipole-coupled molecules and emitter-nano-particle hybrid systems, and down to the single photon level. 



\section{Single molecule optics}

The first optical detection of a single molecule in the solid state dates back to 1989. William E. Moerner and Lothar Kador measured the power of a laser beam transmitted through a p-terphenyl crystal doped with pentacene molecules and cooled to liquid helium temperatures. By using a doublemodulation technique, they were able to observe a faint drop in transmission when the laser frequency was resonant to the transition of a single pentacene molecule [44]. One year later Michel Orrit and Jacques Bernard managed to detect the fluorescence light emitted by a single pentacene molecule [45].

In 1993, Eric Betzig and Robert J. Chichester demonstrated the first measurement of single molecule fluorescence at room temperature [46]. While they excited the molecules with a scanning near field optical microscope to reduce the background, a research group led by Richard N. Zare detected a single molecule with far field optics [47]. This paved the way for superresolution fluorescence microscopy, which nowadays finds widespread applications, especially in biology and biomedical research $[48,49]$. In 2014, William E. Moerner, Eric Betzig and Stefan Hell were awarded with Nobel Price in Chemistry "for the development of super-resolved fluorescence microscopy" [50].

In parallel, single dye molecules became due to their sensitivity for the immediate surrounding an important tool for the investigation of material properties at the nano-scale [51]. Being not limited by ensemble averaging, they could provide insight, e.g., into the disorder of aromatic crystals [52] and Shpol'skii matrices [53].

Stimulated by the favorable photo-physical properties at cryogenic temperatures, in particular a strong and lifetime-limited zero-phonon line and photo-stability over months, organic dye molecules developed also into a promising system for quantum optical research. Already in 1992, it was demonstrated that single molecules emit a stream of single photons by observing the correlations between fluorescence photons of a pentacene molecule [54]. In the following, the discovery of photo-induced, reversible changes of the resonance frequency showed the possibility of optically switching the state of a molecule [55, 56]. In 1995, quantum jumps between 
singlet and triplet states were witnessed as short interruptions in the fluorescence signal from a terrylene molecule embedded in a p-terphenyl crystal [57]. In the same system, coherent interaction of two molecules, mediated by dipole-dipole coupling and producing sub- and super-radiant entangled states, was monitored [58]. Subsequently, significant progress in coupling light and single molecules more efficiently enabled a series of new experiments: the fluorescence triplet as predicted by Mollow [59] was observed for the first time from a single emitter in the solid state [6o] and manipulation of amplitude [61] and phase [62] of a weak laser beam by a single molecule was demonstrated. Further advances were also made regarding the use of dye molecules as light source: The appearance of quantum interferences proved the indistinguishability of photons emitted by two independent molecules [63]. Moreover, a single molecule was employed as light source for the spectroscopy of another molecule [64] and of a cloud of sodium atoms [65].

Several of these experiments [6o-62, 64] investigated the interaction of excitation light and single molecule, by observing the amplitude or phase of the transmitted laser power. To induce a notable change of this signal, the emitter must interact with a significant fraction of the incoming photons. This requires, on the one hand, proper focusing and mode matching of the incoming light to the emitter [66] and depends, on the other hand, on the photo-physical properties of the emitter. While this approach is far more challenging than detecting the fluorescence signal, it allows additional insight by providing access to the coherent interaction of light with a single emitter.

This chapter serves as an introduction to the field of single molecule spectroscopy. In the first section, the aromatic dye dibenzanthanthrene (DBATT) and its optical properties are summarized. Furthermore, the addressing of a single emitter by using a combination of spatial and spectral selectivity is discussed. In addition, the theoretical description of light-matter interaction using the optical Bloch equations is recapitulated and the notation used throughout this thesis is introduced. The second section focuses on the actual experimental realization, including sample preparation, cryostat, and the optical setup. In the third section, standard single molecules characterization measurements, like fluorescence and extinction measurements, are described, while we discuss the detection of molecule pairs and possible experiments with them in the last section. 


\subsection{Basic concepts of single molecule spectroscopy}

\subsubsection{Photo-physical properties of dye molecules in organic matrices}

The electronic ground state $S_{0}$ of a typical dye molecule is a singlet state, where the electrons in the highest occupied molecular orbital are paired to yield a total spin of zero. Via an optical transition, which is usually in the range of visible to near infrared light, it can be transferred into one of several excited states $S_{n}$. For the purpose of this thesis, only the first excited state $S_{1}$ is important, as the other electronic states are sufficiently far away to not interact with the narrow-band excitation light.

The efficiency of light-matter interaction can be approximated by the ratio of the scattering cross section to the effective area of the input beam [26]. Within certain limits, a dye molecule can be described as a two-level system (TLS) with a transition wavelength $\lambda$, which has a scattering cross section of $\sigma_{0}=3 \lambda^{2} / 2 \pi \approx \lambda^{2} / 2$ for light at its resonance frequency [67]. Diffraction limits the minimum focal area of the input beam to roughly $(\lambda / 2)^{2}$, comparable to the scattering cross section of a TLS and thus promising a very efficient interaction.

At ambient conditions however, dye molecules as well as most other emitters in solid-state systems are usually many orders of magnitude away from this perfect value [68]. The difference is mainly caused by coupling of the molecule and the host material inducing a large dephasing rate $\Gamma_{2}$. This increases the homogeneous linewidth $\Gamma_{\text {hom }}$ and reduces the scattering cross section to

$$
\sigma=\sigma_{0} \frac{\Gamma_{1}}{\Gamma_{\text {hom }}},
$$

with $\Gamma_{1}$ being the decay rate of the excited state population. Throughout this thesis, we assume that dephasing is the only source of line broadening which means that $\Gamma_{\text {hom }}=2 \Gamma_{2}$. At room temperature, the factor $\Gamma_{1} / \Gamma_{\text {hom }}$ can be as small as $10^{-6}$ [69]. For certain guest-host combinations, it is possible to reduce dephasing to its fundamental limit of $\Gamma_{2}=\Gamma_{1} / 2$ by cooling the sample to cryogenic temperatures. Under these conditions, the thermal excitation of vibrations in the host material is suppressed and the scattering cross section reaches its ideal value $\sigma_{0}$.

However, even if we consider only the electronic states $S_{0}$ and $S_{1}$, a molecule is still not a true TLS. A usual dye molecule consists of several ten atoms giving rise to numerous vibrational degrees of freedom which introduce extra energy levels (see Fig. 2.1 a). On top of that, the matrix supports 

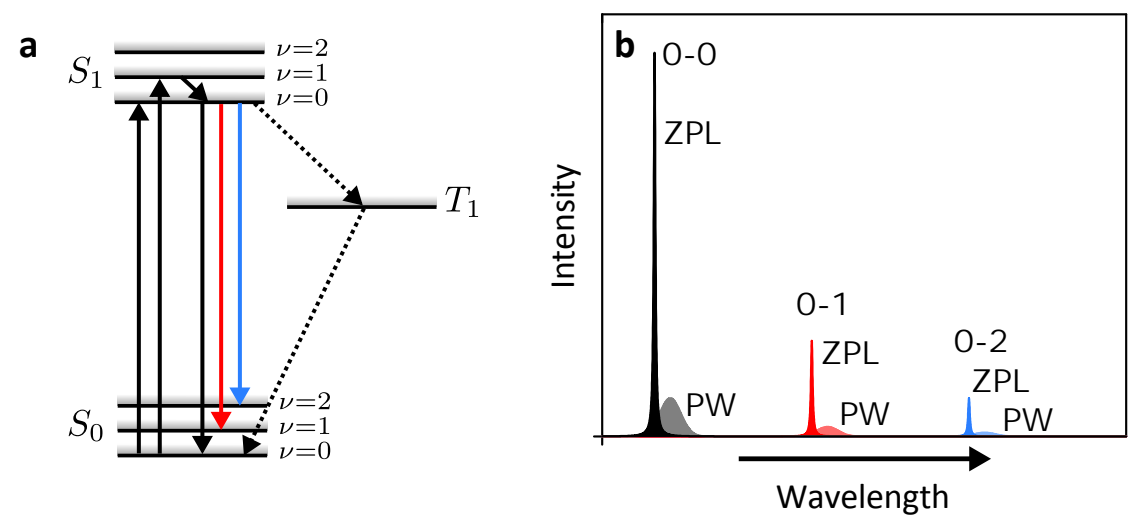

Figure 2.1: a, Jablonsky diagram of a dye molecule. For simplicity only the first three vibration states (labeled with $\nu$ ) are depicted for each electronic level. The arrows indicate the main transitions. The color coding of the $S_{1} \rightarrow S_{0}$ transitions correspond to the colors used in part $\mathbf{b}$. The dotted lines illustrate the slow transition rate into and out of $T_{1}$. $\mathbf{b}$, Simplified fluorescence spectrum of a typical dye molecule at low temperatures. The zero-phonon lines (ZPL) are visible as sharp peaks accompanied by wider phonon wings (PW). Adapted from $[71]$.

a quasi continuum of low energy vibration modes. We account for the influence of these additional decay channels on the scattering cross section by replacing $\Gamma_{1}$ in Eq. (2.1) with the partial decay rate from $S_{1} \rightarrow S_{0}, \Gamma_{S_{1} \rightarrow S_{0}}$. The scattering cross section of the $S_{0} \rightarrow S_{1}$ dipole transition is then given by [70]:

$$
\sigma_{S_{0} \rightarrow S_{1}}=\frac{3 \lambda^{2}}{2 \pi} \frac{\Gamma_{S_{1} \rightarrow S_{0}}}{\Gamma_{\text {hom }}} .
$$

The influence of matrix and molecule vibrations can be nicely seen in the fluorescence spectrum of a dye molecule (see Fig. 2.1 b). The first narrow peak, the o-o zero-phonon line (ZPL), corresponds to the transition from $S_{1}$ to $S_{0}$ without the excitation of vibrations. Excitation of matrix vibrations, with frequencies typically in the range of $30-300 \mathrm{GHz}$ [72], leads to the appearance of a broad phonon wing (PW) next to the ZPL. Intra-molecular vibrations have discrete frequencies in the order of $10 \mathrm{THz}$ and a lifetime of picoseconds [73]. Their excitation leads to a larger red-shift of the emitted photons, and thus the appearance of ZPLs and PWs at longer wavelengths. Experimentally, these fluorescence photons are the standard tool for the detection of a single molecule, as they can be easily separated from the excitation light by using optical long-pass filters. The transitions are labeled by the vibrational quantum numbers of the initial and the final state, e.g., the transition from $S_{1, \nu=0}$ to $S_{0, \nu=2}$ is called the $0-2$ transition. 
The transition probability into a particular vibration state is determined by the overlap of the initial and final vibration state wave functions [74]. The coupling to intra molecular vibrations and matrix phonons is characterized by the Franck-Condon factor, $\alpha_{\mathrm{FC}}$, and the Debye-Waller factor, $\alpha_{\mathrm{DW}}$, respectively. $\alpha_{\mathrm{FC}}$ is defined as

$$
\alpha_{\mathrm{FC}}=\frac{I_{0-0}}{I_{\mathrm{tot}}},
$$

with $I_{0-0}$ being the integrated intensity of the $0-0$ transition and $I_{\text {tot }}$ the total intensity emitted by the molecule. The Debye-Waller factor can be calculated from the integrated intensities of the o-o ZPL, $I_{\mathrm{ZPL}}$, and the corresponding phonon wing, $I_{\mathrm{PW}}$, by

$$
\alpha_{\mathrm{DW}}=\frac{I_{\mathrm{ZPL}}}{I_{\mathrm{ZPL}}+I_{\mathrm{PW}}}
$$

The partial decay rate, $\Gamma_{S_{1} \rightarrow S_{0}}$, is related to the total decay rate, $\Gamma_{1}$, via:

$$
\Gamma_{S_{1} \rightarrow S_{0}}=\underbrace{\alpha_{\mathrm{FC}} \alpha_{\mathrm{DW}}}_{=\alpha} \Gamma_{1} .
$$

Hence, the scattering cross section is reduced compared to an ideal TLS by a factor of $\alpha$ due to the presence of vibrational states.

Beyond vibrational levels, dye molecules also have additional electronic states. Besides the already mentioned spin singlet states, there are manifolds of triplet states $T_{n}$, where the total electron spin equals to one. The transition from a singlet to a triplet state involves a spin flip and is thus dipole-forbidden. Nevertheless, the molecule can decay from $S_{1}$ to $T_{1}$ at the small inter system crossing (ISC) rate. While in the triplet state, the molecule cannot be excited by light fields resonant with the o-o ZPL, until the triplet state population eventually decays into $S_{0}$. As this transition is also dipole-forbidden, the lifetime of $T_{1}$ is typically several orders of magnitude larger than the lifetime of $S_{1}$ [73]. Depending on the ISC rate and the lifetime of $T_{1}$, the triplet state can form a bottleneck severely reducing the interaction of the molecule with an incident laser field.

The resonance frequency of a dye molecule is very sensitive to the nano-environment which makes them excellent local probes for strain [75] or electric fields $[76,77]$ inside a crystal. Imperfections in the crystal cause local deviations of these fields from their average value. Hence, even though the molecules are identical, the resonance frequencies are distributed over a range called the inhomogeneous broadening (see Fig. 2.2), typically on the order of $1 \mathrm{THz}$. At room temperature, this distribution is concealed by 

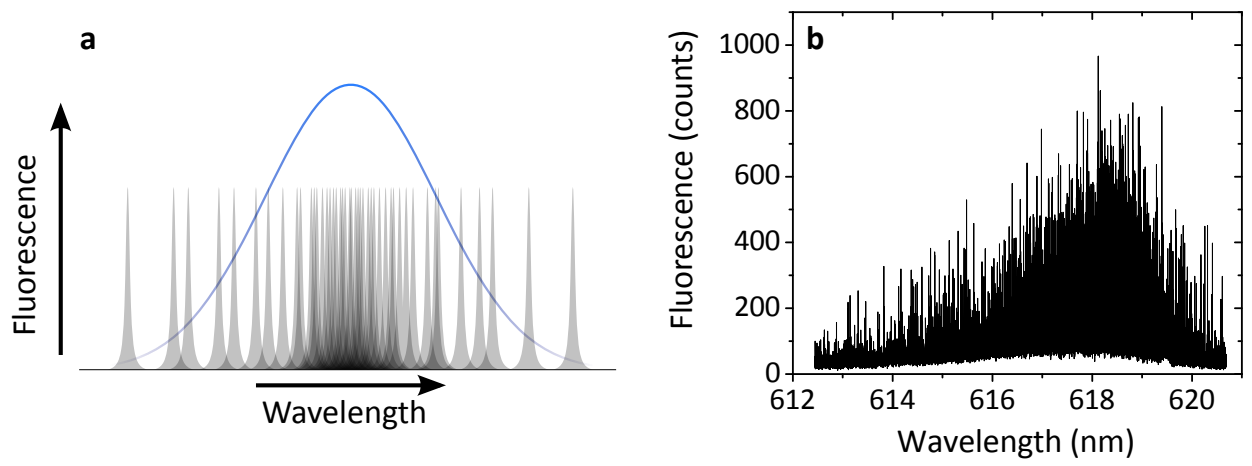

Figure 2.2: a, Schematic distribution of single molecule resonances (gray) over the inhomogeneous broadening (blue curve). Adapted from [78]. b, Fluorescence excitation spectrum of DBATT in naphthalene. Note that even in the densest region, around $618 \mathrm{~nm}$, the spectrum consists of single, well-resolved peaks. Courtsey of Benjamin Gmeiner.

the (homogeneously) broadened transition and becomes only visible at low temperatures, when the linewidth approaches the lifetime limit.

Even at low temperatures, strain and electric fields inside the host material are not necessarily static. Changes of these fields lead to shifts of the resonance frequencies over time which is known as spectral diffusion. The characteristics can be quite versatile: even within the same sample the behavior can range from slow drifts over quasi-continuous wandering to sudden frequency jumps. Molecules with resonance frequencies far from the center of the inhomogeneous broadening have typically a larger probability for spectral diffusion [79].

When choosing a suitable emitter, one has to take all these effects into account. There are numerous combinations of dye molecules and matrices that show lifetime-limited transitions at liquid helium temperature [68]. But the ideal emitter should furthermore have high Debye-Waller and FranckCondon factors, a low ISC rate and show no spectral diffusion.

\subsubsection{Addressing a single molecule}

To perform experiments on a single molecule, we need the ability to selectively excite and collect light from a single emitter. At room temperature this is done by using microscope objectives with a large numerical aperture, to achieve a high spatial resolution. The concentration of dye molecules is then chosen such that less than one molecule is present per focal volume.

At low temperatures, the narrow linewidth of the o-o transition enables spectral selection of single molecules in addition to the spatial selection 
<smiles>c1ccc2c3ccc4cc5cc6ccccc6c6ccc(cc-3cc2c1)c-4c56</smiles>

2.3,8.9-dibenzanthanthrene<smiles>c1ccc2ccccc2c1</smiles>

naphthalene

Figure 2.3: Skeletal formulas of the dye molecule dibenzanthanthrene and the matrix constituent naphthalene.

used at room temperature. As mentioned in the previous subsection, the resonance frequencies are distributed over the inhomogeneous broadening (Fig. 2.2). Thus, two molecules can still be separated even if they are within one focal volume, as long as their resonances do not overlap. While the linewidth is tens of megahertz, the inhomogeneous distribution spans a terahertz or more. This means that even if thousand molecules are within one focus, it is still possible to address them individually by using a narrow-band laser. This is particularly useful if a molecule is required at a specific position, e.g., close to a plasmonic nano-structure [80-83]. The high concentration drastically increases the probability to have a molecule at the desired position.

\subsubsection{DBATT in naphthalene}

Most experiments presented in the following were performed on 2.3,8.9-dibenzanthanthrene (DBATT) molecules embedded in a naphthalene matrix (see Fig. 2.3). This combination offers a lifetime-limited o-o ZPL for temperatures below $4 \mathrm{~K}$. Furthermore, the ISC rate is so low that the triplet state contribution can be neglected [84]. Figure 2.4 a shows the wavelengths of the most important optical transitions. The inhomogeneous distribution of the o-o ZPLs is peaked around $618.7 \mathrm{~nm}$, which is conveniently accessible with a dye laser using Sulforhodamine B as gain medium. The frequency of the o-1 transition is almost exactly $7.7 \mathrm{THz}$ away from the o-o transition. Using that knowledge, it is possible to address the same molecule in o-o and o-1 excitation. The dominant component of the red-shifted fluorescence has a wavelength of $629 \mathrm{~nm}$.

From the fluorescence emission spectrum shown in Fig. 2.4 b, we can determine the Debye-Waller and Franck-Condon factors by integrating the relevant spectral windows. While the exact values differ from molecule to 
molecule, typically both factors are around 0.7 , which means that roughly $50 \%$ of the light is emitted in the o-o ZPL.

The linewidth of a transition can be measured by observing the red-shifted fluorescence as a function of the excitation laser frequency at low excitation powers. Figure 2.4 shows such a measurement for the o-o (c) and the o-1 (d) transition. In case of the o-o transition, we detect a linewidth of $22 \mathrm{MHz}$ corresponding to the lifetime limit [84]. The line shape is in perfect agreement with the expected Lorentzian. Due to the much shorter lifetime of the $S_{1, \nu=1}$ level, the linewidth of the o-1 transition is three orders of magnitude larger. Exciting the molecule via the o-1 transition allows separating the photons emitted at the o-o transition from the excitation light [65]. This is, for example, necessary to measure a complete fluorescence spectrum, as the one presented in Fig. 2.4 b.

While DBATT does not show any bleaching at low temperatures in a variety of matrices (e.g. n-tetradecane [85], n-hexadecane [86], p-terphenyl [87] or methyl methacrylate [88]), it is particularly stable inside a naphthalene matrix. Thus, it displays only very small spectral diffusion, which is especially important for measurements with long integration times. Figure $2.4 \mathrm{e}$ shows a time trace of the fluorescence signal that is recorded with the excitation laser intensity being more than 1000 times above saturation. The molecule does not show any blinking or spectral jumps even at such strong laser driving. Only a small drift is present leading to a standard deviation of the fluorescence signal of twice the shot noise limit.

The combination of these properties makes DBATT in naphthalene an attractive system for studying the interaction of light with a single quantum emitter. Even though it is not a perfect TLS, its strong o-o ZPL allows efficient coupling with light. Furthermore, the spectral stability, even at high excitation powers, enables investigations with strong pump fields and long integration times, like the ones presented in chapter 4 .

\subsubsection{Interaction of light with a single molecule}

"It is impossible to treat even one atom's interaction with light exactly" [89]. This statement by Allen and Eberly emphasizes the necessity of approximations when dealing with light-matter interaction. The usual assumptions are that only a single transition between two matter states interacts with the incoming light, which should be monochromatic with a frequency close to the resonance. In the case of our experiment, both assumptions are valid. The most pronounced transition is the o-o ZPL. As both involved states have zero spin, they do not show any splitting due to external magnetic fields. The 

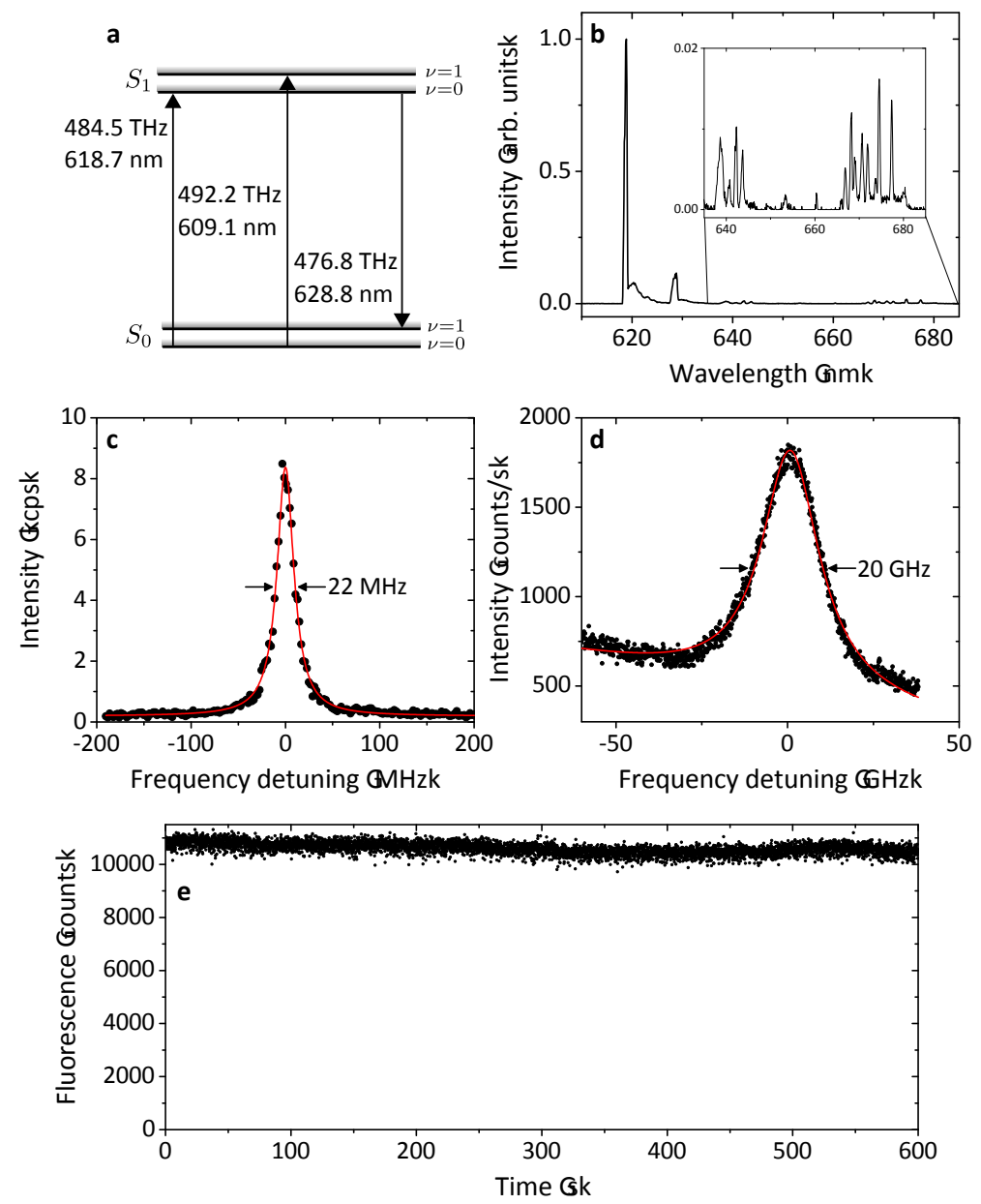

Figure 2.4: a, Jablonsky diagram with the most important energy levels of DBATT in naphthalene. The given transition frequencies refer to a molecule in the center of the inhomogeneous broadening. $\mathbf{b}$, Fluorescence emission spectrum of a single DBATT molecule. The pronounced first peak contains around $50 \%$ of the total emission and corresponds to the o-o ZPL. The inset shows a zoom at the region from $635 \mathrm{~nm}$ to $685 \mathrm{~nm}$. Note that the drop at $680 \mathrm{~nm}$ is caused by an optical filter. Fluorescence excitation spectra for o-o (c) and o-1 (d) excitation. Please note that the frequency axis of plot $\mathbf{c}$ is in $\mathrm{MHz}$ while that of plot $\mathbf{d}$ is in GHz. The solid line is a Lorentzian fit to the data which includes a linear background for plot d. e, Fluorescence time trace recorded with the excitation laser at resonance with a power more than 1000 times above saturation. 
next closest states are vibrational excitations of the molecule, with an energy difference corresponding to several hundred thousand linewidths of the o-o ZPL. Hence, it is possible to address only the $S_{0, \nu=0} \rightarrow S_{1, \nu=0}$ transition with a narrow-band laser. However, the excited state decays with a considerable probability into vibrationally excited states $S_{0, \nu \neq 0}$. This red-shifted fluorescence amounts in our system to roughly $50 \%$ of the emitted light. It leads to a reduction of the transition dipole moment $\mathbf{d}$ by a factor of $\sqrt{\alpha}$. For now, we will assume an ideal TLS and include this effect where necessary in the final equations.

The interaction of a monochromatic light field $\mathbf{E}_{\text {inc }}$ and a TLS at the origin $\mathcal{O}$ can be described in a semi-classical theory and within the dipole approximation using the following Hamiltonian

$$
\hat{H}=\hbar \omega_{0} \hat{\sigma}^{\dagger} \hat{\sigma}-\hat{\mathbf{d}} \cdot \mathbf{E}_{\text {inc }}(\mathcal{O}, t) .
$$

The TLS has a ground state $|g\rangle$ and an excited state $|e\rangle$, which are separated by an energy difference $\hbar \omega_{0} . \hat{\sigma}$ and $\hat{\sigma}^{\dagger}$ are the atomic lowering and raising operators that can be expressed in the basis of the TLS eigenstates as

$$
\hat{\sigma}=|g\rangle\langle e| \quad \text { and } \quad \hat{\sigma}^{\dagger}=|e\rangle\langle g| .
$$

The atomic dipole operator $\hat{\mathbf{d}}$ can be written in terms of $\hat{\sigma}$ and $\hat{\sigma}^{\dagger}$ as

$$
\hat{\mathbf{d}}=\mathbf{d}\left(\hat{\sigma}+\hat{\sigma}^{\dagger}\right), \quad \text { with } \quad \mathbf{d}=\langle e|\hat{\mathbf{d}}| g\rangle=\langle g|\hat{\mathbf{d}}| e\rangle,
$$

where the arbitrary phases of $|g\rangle$ and $|e\rangle$ were chosen such that $\mathbf{d}$ is real. $\mathbf{E}_{\text {inc }}(\mathcal{O}, t)$ is the electric driving field with an angular frequency $\omega$ and an amplitude $\mathbf{E}_{\text {inc }}(\mathcal{O})$ at the position of the TLS. Its time dependence is given by:

$$
\mathbf{E}_{\text {inc }}(\mathcal{O}, t)=\frac{\mathbf{E}_{\text {inc }}(\mathcal{O})}{2}\left(e^{i \omega t}+e^{-i \omega t}\right) .
$$

By using Eq. (2.8) and (2.9) the Hamiltonian [Eq. (2.6)] can be written as

$$
\hat{H}=\hbar \omega_{0} \hat{\sigma}^{\dagger} \hat{\sigma}+\frac{\hbar \Omega}{2}\left(\hat{\sigma} e^{i \omega t}+\hat{\sigma} e^{-i \omega t}+\hat{\sigma}^{\dagger} e^{i \omega t}+\hat{\sigma}^{\dagger} e^{-i \omega t}\right),
$$

where the Rabi frequency $\Omega=-\frac{\mathbf{d E}_{\text {inc }}()}{\hbar}$ was introduced. By changing to a reference frame rotating at the frequency of the electric field, $\omega$, Eq. (2.10) becomes

$$
\hat{\tilde{H}}=-\hbar \Delta \hat{\sigma}^{\dagger} \hat{\sigma}+\underbrace{\frac{\hbar \Omega}{2}\left(\hat{\sigma}+\hat{\sigma}^{\dagger}+\hat{\sigma} e^{-i 2 \omega t}+\hat{\sigma}^{\dagger} e^{i 2 \omega t}\right)}_{\text {interaction part }},
$$


with $\Delta=\omega-\omega_{0}$ being the detuning between incident field and transition frequency. The tilde denotes the fact that we are in the rotating frame. In this reference frame, the interaction part of $\hat{\tilde{H}}$ has components oscillating at $2 \omega$ and parts that are time independent. In the rotating wave approximation (RWA), we ignore the fast oscillating terms, as their net contribution has only an insignificant impact on the system [90]. Nevertheless, on performing this approximation some physical effects are lost, e.g., the Bloch-Siegert shift [89, 91]. But its influence is negligible in the experiment.

The Hamiltonian after the RWA is given by:

$$
\hat{\tilde{H}}_{\mathrm{RWA}}=-\hbar \Delta \hat{\sigma}^{\dagger} \hat{\sigma}+\frac{\hbar \Omega}{2}\left(\hat{\sigma}+\hat{\sigma}^{\dagger}\right) .
$$

Using Eq. (2.12), it is straight forward to calculate the equations of motion for the density matrix $\hat{\tilde{\rho}}$ in the rotating frame using the von Neumann equation:

$$
i \hbar \frac{\partial \hat{\tilde{\rho}}}{\partial t}=[\hat{\tilde{H}}, \hat{\tilde{\rho}}], \quad \text { with } \quad \hat{\tilde{\rho}}=\left(\begin{array}{cc}
\tilde{\rho}_{e e} & \tilde{\rho}_{e g} \\
\tilde{\rho}_{g e} & \tilde{\rho}_{g g}
\end{array}\right) .
$$

The density matrix $\hat{\rho}$ in the rest frame can then be obtained from $\hat{\tilde{\rho}}$ by transforming the latter back into the initial frame. This yields the following relation between the respective matrix elements:

$$
\hat{\rho}=\left(\begin{array}{ll}
\tilde{\rho}_{e e} & \tilde{\rho}_{e g} e^{-i \omega t} \\
\tilde{\rho}_{g e} e^{i \omega t} & \tilde{\rho}_{g g}
\end{array}\right) .
$$

The elements $\tilde{\rho}_{e e}$ and $\tilde{\rho}_{g g}$ describe the probability of the TLS to be in the excited or in the ground state, respectively. To interpret the off-diagonal elements $\tilde{\rho}_{e g}=\tilde{\rho}_{g e}^{\star}$, it is useful to look at the expectation value of the dipole operator:

$$
\langle\hat{\mathbf{d}}\rangle=\mathbf{d} \operatorname{Tr}\left(\hat{\rho}\left(\hat{\sigma}+\hat{\sigma}^{\dagger}\right)\right)=\mathbf{d}\left(\operatorname{Re}\left(\tilde{\rho}_{g e}\right) \cos (\omega t)-\operatorname{Im}\left(\tilde{\rho}_{g e}\right) \sin (\omega t)\right) .
$$

Hence, the real and imaginary parts of $\tilde{\rho}_{\text {eg }}$ are related to the in-phase and in-quadrature parts of the dipole moment, respectively [9o].

Before writing down the differential equations from Eq. (2.13) explicitly, it is convenient to introduce the Bloch vector $\mathbf{x}$. Its components $u, v$ and $w$ describe the real and imaginary parts of $\tilde{\rho}_{\text {ge }}$ and the population inversion, i.e., the difference between the population in the excited and in the ground state: 


$$
\begin{aligned}
\mathbf{x} & =\left(\begin{array}{c}
u \\
v \\
w
\end{array}\right), \\
u & =\frac{1}{2}\left(\tilde{\rho}_{g e}+\tilde{\rho}_{e g}\right), \\
v & =\frac{1}{2 i}\left(\tilde{\rho}_{g e}-\tilde{\rho}_{e g}\right), \\
w & =\frac{1}{2}\left(\tilde{\rho}_{e e}-\tilde{\rho}_{g g}\right) .
\end{aligned}
$$

Using a classical field for this calculation omits spontaneous decay, which is a result of the coupling to the vacuum field [7o]. Thus, it is necessary to add this effect by introducing a decay rate $\Gamma_{1}$ for the excited state and $\Gamma_{2}$ for the dipole moment. The resulting set of coupled first order linear differential equations, known as optical Bloch equations, is given by $[89,90]$ :

$$
\begin{aligned}
\dot{u} & =\Delta v-\Gamma_{2} u, \\
\dot{v} & =-\Delta u-\Omega w-\Gamma_{2} v, \\
\dot{w} & =\Omega v-\Gamma_{1} w-\Gamma_{1} / 2 .
\end{aligned}
$$

Many spectroscopic observations can be described by looking at their steadystate solution, i.e., $\dot{u}=\dot{v}=\dot{w}=0$. Those solutions are:

$$
\begin{aligned}
& u_{\mathrm{st}}=\frac{\Delta \Omega}{2\left(\Delta^{2}+\Omega^{2} \frac{\Gamma_{2}}{\Gamma_{1}}+\Gamma_{2}^{2}\right)}=\frac{\Delta \Gamma_{1}}{2 \Omega \Gamma_{2}} \frac{S}{S+1}, \\
& v_{\mathrm{st}}=\frac{\Gamma_{2} \Omega}{2\left(\Delta^{2}+\Omega^{2} \frac{\Gamma_{2}}{\Gamma_{1}}+\Gamma_{2}^{2}\right)}=\frac{\Gamma_{1}}{2 \Omega} \frac{S}{S+1}, \\
& w_{\mathrm{st}}=\frac{\Gamma_{2} \Omega^{2}}{2 \Gamma_{1}\left(\Delta^{2}+\Omega^{2} \frac{\Gamma_{2}}{\Gamma_{1}}+\Gamma_{2}^{2}\right)}-\frac{1}{2}=\frac{1}{2} \frac{S}{S+1}-\frac{1}{2} .
\end{aligned}
$$

Where we introduced the saturation parameter:

$$
S=\frac{\Omega^{2} \frac{\Gamma_{2}}{\Gamma_{1}}}{\Delta^{2}+\Gamma_{2}^{2}} .
$$

It is a measure for the driving strength of the incident light field and allows comparison of excitation intensities in different systems. It scales quadratically with the Rabi frequency and hence linearly with the intensity of the incident field. In the experiment, we often deal with the case of a lifetimelimited emitter, i.e., $\Gamma_{2}=\Gamma_{1} / 2$, and resonant excitation, i.e., $\Delta=0$. In that case, Eq. (2.25) simplifies to: 


$$
S=2 \frac{\Omega^{2}}{\Gamma_{1}^{2}}
$$

\subsubsection{Fluorescence}

Upon excitation, a molecule emits photons at a rate $R_{\text {fluo }}$ proportional to the population in the excited state $\rho_{\mathrm{ee}}$ :

$$
R_{\text {fluo }}=\Gamma_{1} \rho_{\text {ee }}=\Gamma_{1} \frac{S}{S+1} \frac{1}{2} .
$$

The equation shows that the rate of emitted photons is limited to $\Gamma_{1} / 2$ for a single emitter. This behavior is an immediate consequence of the two discrete energy levels. If the system is in the upper state, it cannot be excited anymore and needs a time of $1 / \Gamma_{1}$ to relax into the ground state. Hence, the lifetime of the excited state becomes a bottleneck for light emission that cannot be overcome by increasing the pump power. To obtain more light from the emitter one needs to change its decay rate. Following Fermi's Golden rule, this requires a change of the local density of states, e.g., by using an optical antenna $[81,92]$ or by placing the emitter inside a cavity $[93,94]$. Note that the factor $\frac{1}{2}$ in Eq. (2.27) follows from the resonant pumping scheme and can be overcome for emitters with a suitable multi-level scheme. For dye molecules, it is possible to excite into a short lived vibrational level of the excited state. In that way, it is possible to bring the whole population into the excited state, thus increasing the maximum emission rate by a factor of two.

If one considers the emission rate as function of the detuning $\Delta$, one can see from Eq. (2.24) that the dependence is given by a Lorentzian with a full width at half maximum (FWHM) of:

$$
\Gamma_{\mathrm{FWHM}}=2 \sqrt{\Omega^{2} \frac{\Gamma_{2}}{\Gamma_{1}}+\Gamma_{2}^{2}} \underset{\Delta=0}{=} 2 \Gamma_{2} \sqrt{S+1} .
$$

In case of a very weak driving field, the linewidth is given by $2 \Gamma_{2}$, which is equal to $\Gamma_{1}$ in the absence of pure dephasing. For stronger excitation, power broadening leads to an increase of the linewidth. Both features, saturation of the emitted fluorescence light as well as line broadening, can be experimentally observed (see Fig. (2.5)). From both curves, one can determine a relation between incident laser power and Rabi frequency. We define the saturation power $P_{S}$ as the power of the incident field required to drive a TLS on resonance with a saturation parameter of $S=1$ (i.e., $\Omega=\Gamma_{1} / \sqrt{2}$ ). In chapter 3, we use $P_{S}$ as a figure of merit to describe the coupling efficiency between light and emitter. 

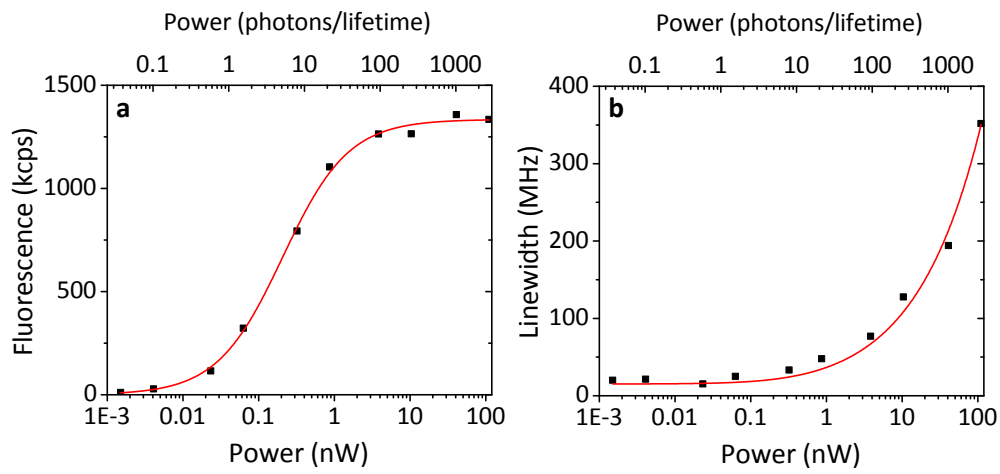

Figure 2.5: Saturation measurement of a single molecule. For excitation powers distributed over five orders of magnitude, fluorescence excitation spectra are recorded. From these, we extract the peak fluorescence count rate (a) and the linewidth (b). The black squares are measured values and the red lines show a simultaneous fit of Eq. (2.27) and (2.28) to the data. The power is corrected for the transmission of the elements inside the cryostat and thus corresponds to the excitation power at the sample. The fit yields a saturation power of $208 \mathrm{pW}$ or 5 photons per lifetime.

One interesting feature of TLS emission is the fact that two photons are never emitted at the same time. This purely quantum mechanical phenomenon is known as anti-bunching. It can be qualitatively understood from a heuristic point of view: After the emission of a photon the TLS is in the ground state. From there it needs some time to be re-excited and to decay under the emission of another photon. Hence, it is impossible for the TLS to directly emit two photons. For commonly used dye molecules this time delay is in the range of nanoseconds.

The second order correlation function $g^{(2)}(\tau)$ can be used to describe this behavior. It specifies the probability to detect a photon at a time $t+\tau$ under the condition that a photon has been detected at time $t$. In the case of resonant excitation and a lifetime-limited emitter, i.e., $\Delta=0$ and $\Gamma_{2}=\Gamma_{1} / 2$, it is given by [95]:

$$
\begin{gathered}
g^{(2)}(\tau)=1-\left(\cos (\mu \tau)+\frac{3 \Gamma_{1}}{4 \mu} \sin (\mu \tau)\right) e^{-3 \Gamma_{1} \tau / 4}, \\
\text { with } \mu=\sqrt{\Omega^{2}-\frac{\Gamma_{1}^{2}}{16}} .
\end{gathered}
$$

The shape of the correlation function is mainly determined by the ratio of the Rabi frequency $\Omega$ and decay rate $\Gamma_{1}$ (see Fig. 2.6 a). In the case of small $\Omega, g^{(2)}(\tau)$ is monotonically increasing from 0 to 1 . When $\Omega$ is increased, 

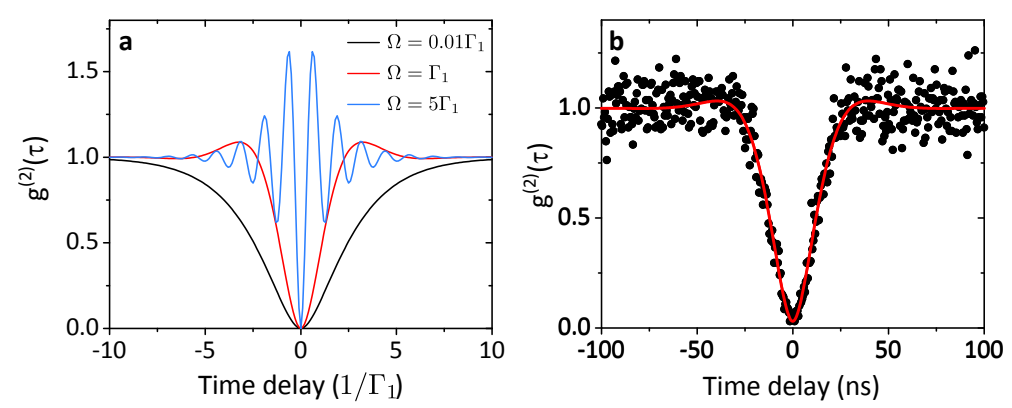

Figure 2.6: a, Theory curves of the second order correlation function for different Rabi frequencies. $\mathbf{b}$, Measurement of the second order correlation function of the fluorescence light of a single DBATT molecule. The red curve is a fit to the data yielding $g^{(2)}(0)=0.03$. No background was subtracted.

Rabi oscillations start to appear and the slope around zero time delay increases. Finally, several oscillation periods become visible at high driving strengths. Measuring this correlation function is the most direct proof for a single-photon emitter. Figure $2.6 \mathbf{b}$ displays such a measurement on the fluorescence of a single DBATT molecule embedded in a n-tetradecane matrix. In that case, $\Omega$ is chosen such that a small onset of the first Rabi oscillation is visible.

\subsubsection{Extinction}

The presence of an emitter in a tight focus can lead to a reduction of the transmitted excitation light which is called extinction. It has two contributions: elastic scattering and absorption by the emitter. A perfect TLS has no absorption, hence, extinction is only caused by interference of the excitation field with that scattered by the emitter in this situation. In case of a molecule, however, red-shifted fluorescence acts as a loss channel, leading to an extinction signal that is a combination of scattering and absorption. In contrast to the fluorescence signal, extinction is sensitive to the phase relation of the involved fields. This method thus probes the coherent interaction of an emitter and the incident light. Indeed, the first signal from a single molecule was detected by observing the extinction signal, although somewhat indirect by using a double-modulation lock-in detection [44].

The impact of a single emitter on the amplitude $[60,71]$ and phase $[62,96]$ of the excitation light is described in detail in previous works. However, as it provides the basis for the description of the coherent, nonlinear interaction 
of a single molecule and two laser beams (see chapter 4), the derivation of the extinction signal is repeated in the following paragraphs.

In a standard extinction measurement, the excitation light is focused onto a single molecule and the transmitted light is observed with a photodetector. The transmitted field $\mathbf{E}_{\text {trans }}$ is the sum of the incident field $\mathbf{E}_{\text {inc }}$ and the field scattered off the molecule $\hat{\mathbf{E}}_{\text {scat }}$. Thus, we can write the intensity $I_{\text {trans }}(\mathbf{r})$ at a position $\mathbf{r}$ in the detection plane as

$$
\begin{gathered}
I_{\text {trans }}(\mathbf{r}) / 2 \epsilon_{0} c=\left\langle\left(\mathbf{E}_{\text {inc }}^{-}(\mathbf{r}, t)+\mathbf{E}_{\text {scat }}^{-}(\mathbf{r}, t)\right)\left(\mathbf{E}_{\text {inc }}^{+}(\mathbf{r}, t)+\mathbf{E}_{\text {scat }}^{+}(\mathbf{r}, t)\right)\right\rangle \\
=\left\langle\mathbf{E}_{\text {inc }}^{-}(\mathbf{r}, t) \mathbf{E}_{\text {inc }}^{+}(\mathbf{r}, t)\right\rangle+\left\langle\mathbf{E}_{\text {scat }}^{-}(\mathbf{r}, t) \mathbf{E}_{\text {scat }}^{+}(\mathbf{r}, t)\right\rangle+ \\
\left\langle 2 \operatorname{Re}\left(\mathbf{E}_{\text {inc }}^{+}(\mathbf{r}, t) \mathbf{E}_{\text {scat }}^{-}(\mathbf{r}, t)\right)\right\rangle
\end{gathered}
$$

where we split the fields in positive and negative frequency parts according to:

$$
\begin{gathered}
\mathbf{E}(\mathbf{r}, t)=\mathbf{E}^{-}(\mathbf{r}, t)+\mathbf{E}^{+}(\mathbf{r}, t) \\
\text { with } \quad \mathbf{E}^{-}(\mathbf{r}, t)=\frac{\mathbf{E}(\mathbf{r})}{2} e^{i \omega t} \quad \text { and } \quad \mathbf{E}^{+}(\mathbf{r}, t)=\mathbf{E}^{-}(\mathbf{r}, t)^{\dagger} .
\end{gathered}
$$

The angled brackets denote the quantum mechanical expectation value and time average. The first and second terms in Eq. (2.30) refer to the intensity of excitation and scattered light, respectively. The third term contains the interference of the two fields. The detected signal $P_{\text {trans }}$ is obtained by integrating the transmitted intensity $I_{\text {trans }}$ over the detection solid angle $A_{\text {det }}$ :

$$
P_{\text {trans }}=\int_{A_{\text {det }}} I_{\text {trans }}(\mathbf{r}) \mathrm{d} A .
$$

For the evaluation of Eq. (2.32), a knowledge of the spatial distribution of the incident and scattered field is required. We describe the spatial variation of the excitation field with a mode function $\mathbf{g}(\mathbf{r})$ that describes amplitude and polarization of the incident field at any position $\mathbf{r}$ [71]. Using that, we write

$$
\mathbf{E}_{\text {inc }}^{-}(\mathbf{r}, t)=\mathbf{g}(\mathbf{r}) E_{\text {inc }}(\mathcal{O}) e^{i \omega t},
$$

with $E_{\text {inc }}(\mathcal{O})=\left|\mathbf{E}_{\text {inc }}(\mathcal{O})\right|$. The function $\mathbf{g}(\mathbf{r})$ contains all information about propagation of the incident field through the optical system. It depends on the focusing optics and the sample geometry.

The scattered field of a TLS in the far field is given by [70]:

$$
\hat{\mathbf{E}}_{\text {scat }}^{-}(\mathbf{r}, t)=\frac{k^{2}|\mathbf{d}|}{4 \pi \epsilon_{0}}\left(\frac{\mathbf{r}}{|\mathbf{r}|} \times \frac{\mathbf{d}}{|\mathbf{d}|}\right) \times \frac{\mathbf{r}}{|\mathbf{r}|} \frac{e^{-i k|\mathbf{r}|}}{|\mathbf{r}|} \hat{\sigma}^{\dagger}(t),
$$


with the wave number $k=\omega / c$. Equation (2.34) can be transformed into a more convenient form by using the Einstein A coefficient, which is equal to the decay rate [7o]

$$
\Gamma_{1}=\frac{|\mathbf{d}|^{2} k^{3}}{3 \pi \epsilon_{0} \hbar}
$$

and the definition of the Rabi frequency for the case $\mathbf{E}_{\text {inc }}(\mathcal{O}) \| \mathbf{d}$. As only the elastically scattered light can interfere with the excitation light, we have to consider the effect of the Franck-Condon and Debye-Waller factors. They reduce the dipole moment of the molecule by $\sqrt{\alpha}=\sqrt{\alpha_{\mathrm{DW}} \alpha_{\mathrm{FC}}}$. The resulting expression for the scattered field is

$$
\hat{\mathbf{E}}_{\text {scat }}^{-}(\mathbf{r}, t)=-\alpha \frac{E_{\text {inc }}(\mathcal{O}) \Gamma_{1}}{\Omega} \underbrace{\frac{3}{4}\left(\frac{\mathbf{r}}{|\mathbf{r}|} \times \frac{\mathbf{d}}{|\mathbf{d}|}\right) \times \frac{\mathbf{r}}{|\mathbf{r}|} \frac{e^{-i k r}}{k|\mathbf{r}|}}_{\mathbf{f}(\mathbf{r})} \hat{\sigma}^{\dagger}(t),
$$

where a similar mode function $\mathbf{f}(\mathbf{r})$ can be introduced to describe amplitude and polarization.

By using Eqs. (2.30), (2.33) and (2.36), we can write Eq. (2.32) as

$$
\begin{aligned}
P_{\text {trans }}=2 c \epsilon_{0} E_{\text {inc }}^{2}(\mathcal{O}) & \left(\int_{A_{\operatorname{det}}}|\mathbf{g}(\mathbf{r})|^{2} \mathrm{~d} A\right. \\
& +\alpha^{2} \frac{\Gamma_{1}^{2}}{\Omega^{2}}\left\langle\hat{\sigma}^{\dagger}(t) \hat{\sigma}(t)\right\rangle \int_{A_{\operatorname{det}}}|\mathbf{f}(\mathbf{r})|^{2} \mathrm{~d} A \\
& \left.-2 \alpha \frac{\Gamma_{1}}{\Omega} \operatorname{Re}\left(\left\langle\hat{\sigma}^{\dagger}(t) e^{-i \omega t}\right\rangle \int_{A_{\operatorname{det}}} \mathbf{f}(\mathbf{r}) \mathbf{g}^{\star}(\mathbf{r}) \mathrm{d} A\right)\right) .
\end{aligned}
$$

Usually, we are not interested in the absolute transmitted power, but rather in the relative change caused by the molecule. Hence, we normalize Eq. (2.37) with the detected excitation power:

$$
P_{0}=2 c \epsilon_{0} E_{\text {inc }}^{2}(\mathcal{O}) \int_{A_{\mathrm{det}}}|\mathbf{g}(\mathbf{r})|^{2} \mathrm{~d} A .
$$

This yields the transmission:

$$
T=\frac{P_{\text {trans }}}{P_{0}}=1+\eta_{\text {mol }} \frac{\Gamma_{1}^{2}}{\Omega^{2}}\left\langle\hat{\sigma}^{\dagger}(t) \hat{\sigma}(t)\right\rangle-2 \frac{\Gamma_{1}}{\Omega} \operatorname{Re}\left(-i \eta e^{i \phi}\left\langle\hat{\sigma}^{\dagger}(t) e^{-i \omega t}\right\rangle\right) .
$$


Here, we introduced two efficiency factors:

$$
\begin{aligned}
& \eta_{\mathrm{mol}}=\alpha^{2} \frac{\int_{A_{\mathrm{det}}}|\mathbf{f}(\mathbf{r})|^{2} \mathrm{~d} A}{\int_{A_{\operatorname{det}}}|\mathbf{g}(\mathbf{r})|^{2} \mathrm{~d} A} \text { and } \\
& \eta=i e^{-i \phi} \alpha \frac{\int_{A_{\operatorname{det}}} \mathbf{f}(\mathbf{r}) \mathbf{g}^{\star}(\mathbf{r}) \mathrm{d} A}{\int_{A_{\operatorname{det}}}|\mathbf{g}(\mathbf{r})|^{2} \mathrm{~d} A}
\end{aligned}
$$

Both factors depend on the spatial distribution of incident and scattered fields. The factor $\eta_{\mathrm{mol}}$ is proportional to the collection efficiency for the light emitted by the molecule, while $\eta$ describes the mode overlap of excitation and scattered light. Furthermore, they include the influence of the branching ratio to the vibrational states and are normalized by the collection efficiency of the incident light. If the molecule is perfectly in focus, $a-\pi / 2$ Gouy phase shift is accumulated in the far field between the focused excitation and the scattered field ${ }^{1}$. In the experiment, a small offset between focus and molecule can cause a deviation $\phi$ from the ideal $-\pi / 2$ phase shift. We account for that with the factor $i e^{-i \phi}$ in Eq. (2.41) which ensures that $\eta$ is a positive, real number.

If the excitation field has the mode of a directional dipole wave, $\eta_{\mathrm{mol}}=\eta^{2}$ [96]. Even though we are not using such a dipolar wave for the excitation in our experiment, the difference between a directional dipole mode and a focused plane wave only becomes visible for focusing optics with opening angles larger than $55^{\circ}[66]$. For our experiment this angle is just $33^{\circ}$, which justifies the approximation $\eta_{\text {mol }} \approx \eta^{2}$.

If the molecule is in the steady state, we can replace the expectation values with the respective density matrix elements and obtain $\left\langle\hat{\sigma}^{\dagger}(t) \hat{\sigma}(t)\right\rangle=\rho_{\text {ee }}$ and $\left\langle\hat{\sigma}^{\dagger}(t) e^{-i \omega t}\right\rangle$

$=\tilde{\rho}_{\mathrm{ge}}$. Using that and the aforementioned approximation, we can write Eq. (2.39) as [96]:

$$
T=1+\eta^{2} \frac{\Gamma_{1}^{2}}{\Omega^{2}} \rho_{\mathrm{ee}}-2 \eta \frac{\Gamma_{1}}{\Omega} \operatorname{Im}\left(\tilde{\rho}_{\mathrm{ge}} e^{i \phi}\right) .
$$

The values of $\eta_{\text {mol }}$ and $\eta$ can be calculated analytically for special cases, like a plane wave focused onto a dipole in free space $[66,97]$. They reach their maximum value for a directional dipole wave incident from an opening angle of $90^{\circ}$, i.e., the excitation light is focused from a full half space onto the molecule. In that case, $\eta=\alpha$ and $\eta_{\text {mol }}=\alpha^{2}$. For a true TLS, we have $\alpha=1$

\footnotetext{
${ }^{1}$ See [96] for a discussion of the origin of this phase shift in case of limited detection angles.
} 

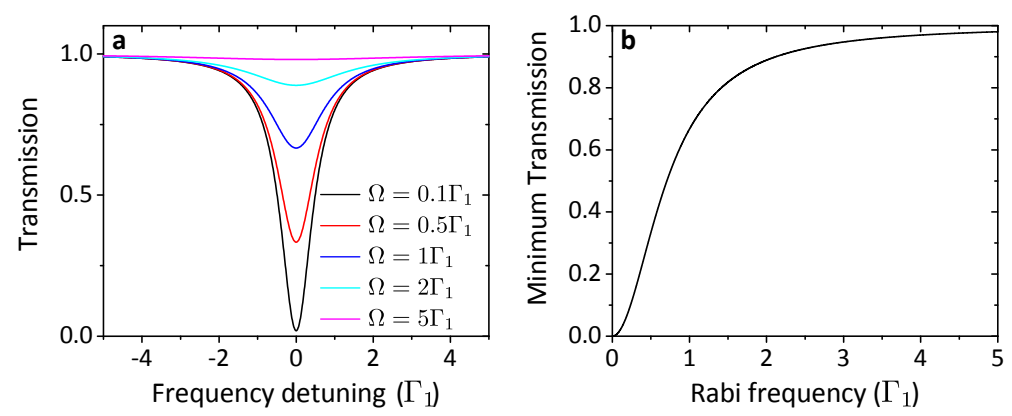

Figure 2.7: a, Transmission as a function of probe laser detuning for different Rabi frequencies in the case of $\eta=1$. From an initial dip that almost goes down to zero the extinction signal becomes less and less visible. $\mathbf{b}$, Transmission at zero detuning as a function of the Rabi frequency.

and hence $\eta=\eta_{\text {mol }}=1$. Figure 2.7 displays extinction curves for different incident powers in this regime. For weak excitation, the transmission signal drops to 0 and all incoming light is reflected by the emitter. With increasing excitation intensity, the visibility of the extinction dip decreases as more light is scattered incoherently and thus cannot interfere with the incident beam.

In case of our experiment, the complex geometry prevents us from knowing $\mathbf{g}(\mathbf{r})$ and $\mathbf{f}(\mathbf{r})$ exactly. Thus, we cannot calculate $\eta$ and use it as a fitting parameter. We estimate its value to be around 0.05 , which corresponds to a $10 \%$ dip in the transmitted intensity. This has two main reasons. First, the dipole moment of a DBATT molecule is reduced by the Debye-Waller and Franck-Condon factor to $\sqrt{\alpha} \mathbf{d}$, which puts an upper limit to the maximum possible $\eta$ of roughly 0.5 . Furthermore, we do not focus a dipole wave onto the molecule and the opening angle of our focusing optics is $33^{\circ}$. Both reduce the mode overlap and thus the extinction signal. Assuming a linearly polarized plane wave, focused onto a TLS with a dipole moment parallel to the polarization of the incident wave, we can use the formulas given in Refs. [66, 98] to calculate $\eta$. Within this simplified model, the limited numerical aperture of the focusing optics leads to $\eta=0.22$, or if we include the branching ratio of the molecule, $\eta=0.11$. In section 3.2, we compare the predictions from this model with measurement results and discuss the reasons for deviations between experiment and theory. 


\subsection{Experimental implementation}

The experimental implementation of an efficient interaction between a light field and a molecule is a technological challenge. A tunable narrow-band laser needs to be tightly focused onto a thin, DBATT-doped naphthalene crystal. This crystal is cooled down to less than $2 \mathrm{~K}$ in a Helium bath cryostat. Transmission and reflection signals are then detected by single photon counting modules (SPCM), a sensitive CCD camera or a spectrometer, depending on the kind of measurement.

This section introduces the necessary experimental techniques. The origin of this setup dates back more than 15 years and was used in the meantime for several doctoral studies [71, 96, 99-101], and the reader is referred to the aforementioned theses for more details.

\subsubsection{Optical setup}

The setup is distributed over two floated optical tables. Both tables are housed with black plastic sliding windows shielding the detectors from ambient light. A filter fan unit, placed above the table, provides a steady flow of filtered air leading to an almost dust free environment on the table. The airflow on the table with the laser system is temperature stabilized to $\pm 0.1 \mathrm{~K}$ reducing possible drifts of the laser cavity.

The light source is a Coherent 899-29 ring dye laser with an autoscan unit. To have tunable emission covering the range of the o-o and o-1 excitation, as well as the red-shifted fluorescence, i.e., $609 \mathrm{~nm}-629 \mathrm{~nm}$, the laser is operated with Sulforhodamine B (Sigma Aldrich) as gain medium. The dye solution is prepared according to the manual. The solvent, ethylene glycol, is hygroscopic which leads to an increasing concentration of water in the solution over time which affects the viscosity. Furthermore, the dye slowly bleaches from irradiation with the $532 \mathrm{~nm}$ pump light. Both effects lead to a degradation of the dye solution, necessitating a dye exchange every other month.

The laser can continuously scan the output frequency over $30 \mathrm{GHz}$ within $250 \mathrm{~ms}$. For larger scans, the autoscan unit with a built-in wavelength meter automatically adjusts the frequency selective elements inside the laser cavity such that the next $30 \mathrm{GHz}$ window has some overlap with the previous one. This stitching technique enables scans over several terahertz with a megahertz resolution. Nowadays, dye lasers have been mostly replaced with more user friendly diode lasers. However, the spectral range of our experiment is still not covered by other laser systems. 

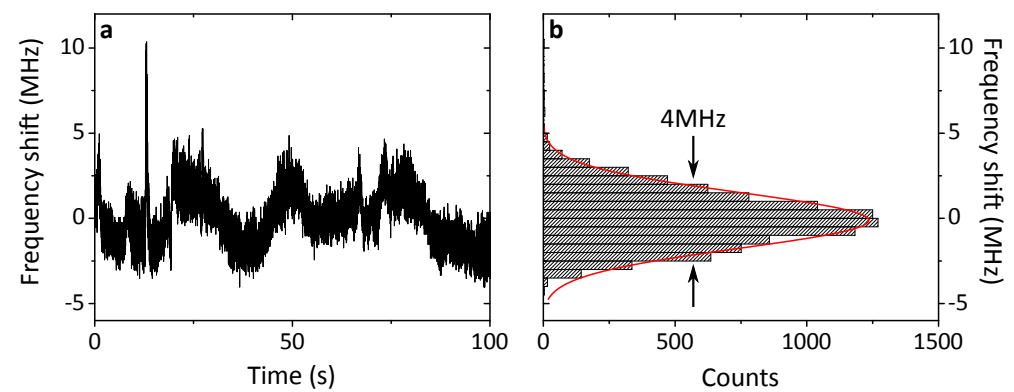

Figure 2.8: a, Measurement of the laser frequency shift over $100 \mathrm{~s}$ with a frequency-stabilized invar cavity. b, Histogram of the frequency jitter with a Gaussian fit (red line). The fit has a FWHM of $4 \mathrm{MHz}$. If the same analysis is done for a time frame of $1 \mathrm{~s}$ the FWHM reduces to $2 \mathrm{MHz}$.

The specification for the laser linewidth is $1 \mathrm{MHz}$. We measured the frequency jitter by monitoring the transmission through a home-built optical cavity. To suppress thermal drifts of the cavity transmission frequency, the cavity length was stabilized using a frequency-stabilized Helium-Neon laser. Figure 2.8 shows the change of the laser frequency over $100 \mathrm{~s}$. The FWHM of the distribution is $4 \mathrm{MHz}$. Within one second, which is the usual time frame of most of the measurements, the jitter amounts to $2 \mathrm{MHz}$ which is well below the linewidth of DBATT in naphthalene.

Figure 2.9 shows a schematic of the optical setup. Light from the ring dye laser passes a neutral density filter, is focused onto an acousto-optical modulator (AOM), and the first diffraction order is coupled into a polarizationmaintaining, single-mode fiber. A half-wave plate is used to match the polarization of the light with the fast or slow axis of the optical fiber. The AOM is part of a feedback system that suppresses fluctuations of the laser power. The amplitude of the radio frequency (rf) field driving the AOM is adjusted by a home-built PID control-loop feedback that receives its reference signal from a photodiode measuring the laser power on the experiment table. The intensity of the light in the first diffraction order follows the changes of the rf amplitude. The neutral density filter is used to adjust the optical power to reach the optimal working point of the feedback system. After this feedback loop, the remaining power fluctuations are $\leq 0.4 \%$ and a Fourier transform does not show any dominant features between $0 \mathrm{~Hz}$ and $500 \mathrm{~Hz}$.

The single-mode fiber guides the light to the second optical table where a cryostat is mounted. A 1ox objective is used to recollimate the beam after the fiber. The resulting beam diameter is larger than the aperture of the focusing asphere inside the cryostat to benefit from the entire numerical aperture 


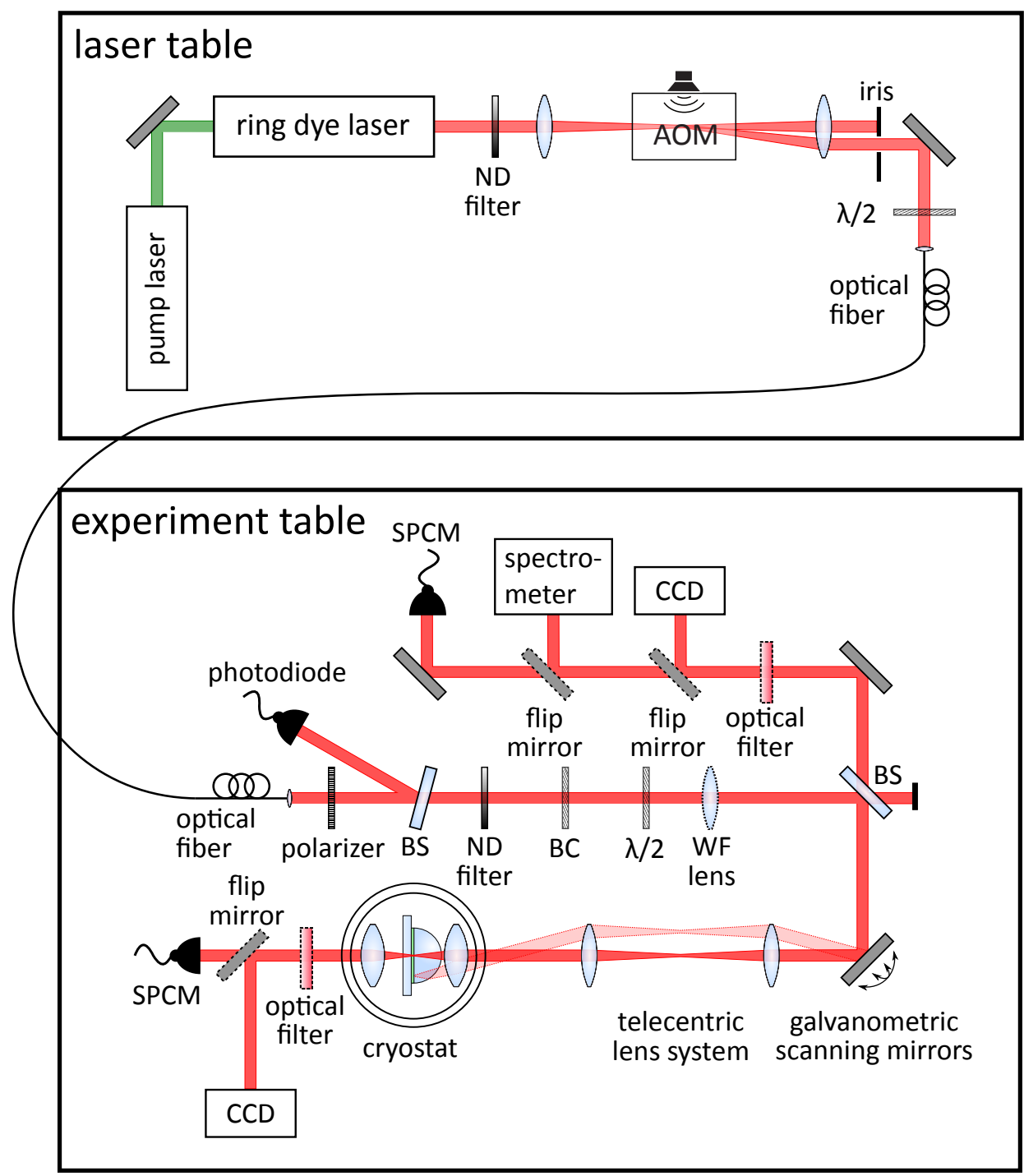

Figure 2.9: Schematics of the experimental setup: the upper part shows the laser table which is temperature stabilized to $\pm 0.1 \mathrm{~K}$. The lower part displays the experiment, with sample, cryostat, and detection. A dashed outline indicates elements that can be removed or inserted depending on the measurement. A detailed explanation is given in the text. The following abbreviations are used: ND filter: optical neutral density filter, AOM: acousto-optical modulator, $\lambda / 2$ : half-wave plate, BS: beam splitter, BC: Berek compensator, WF lens: wide-field lens, SPCM: single photon counting module, CCD: charge coupled device camera. 
(NA) of the lens. After that, a polarizer ensures well-defined linear polarization, and a beam splitter reflects a small fraction of the light onto the reference photodiode for the PID feedback system. Two sets of motorized neutral density filters control the excitation intensity. Subsequently, the combination of a half-wave plate and a Berek compensator (BC) is used to match the polarization of the excitation beam to the dipole orientation of the molecule. The advantage of a BC compared to a quarter-wave plate is its ability to compensate birefringence independently of the input polarization. Hence, once the $\mathrm{BC}$ is adjusted, the polarization can be turned by rotating the half-wave plate without introducing ellipticity.

We direct the light reflected from a beam-splitter, containing $3 \%$ of the incident power, onto a pair of galvanometric scanning mirrors. A telecentric lens pair creates an image of the scanning mirror plane in the back focal plane of the focusing aspheric lens inside the cryostat. A tilt of the scanning mirrors thus changes the beam angle in the back focal plane of the aspheric lens, leading to a lateral displacement of the focal spot on the sample.

The transmitted light is recollimated by a second aspheric lens and can be measured with a CCD camera (Andor Luca) or a SPCM (Perkin Elmer). Light scattered backwards, including a part of the light emitted by the molecule, is recollimated by the first aspheric lens and detected with a SPCM (Perkin Elmer), a CCD camera (Hamamatsu Orca), or a fiber coupled grating spectrometer (Andor Shamrock). Motorized flip mirrors (Newport) are used to switch between different measurement modes. In both detection paths - reflection and transmission - different optical interference filters are used to either suppress the excitation light and detect the red-shifted fluorescence, or to select only the o-o ZPL contribution.

\subsubsection{Cryostat}

The sample and the focusing optics are mounted inside a liquid helium (LHe) cooled cryostat. It is equipped with two tanks connected via a cold needle valve. The upper tank (UT) can be refilled automatically from a helium dewar, while the lower tank (LT) is filled through the needle valve. The average LHe consumption is $250 \mathrm{~L}$ per week and the amount of evaporated helium is monitored with a gas meter. This serves as an indicator for insulation problems of the cryostat which would lead to an increased evaporation rate.

The cryostat has four thermal shields, two are at room temperature, one is in thermal contact with the UT, and the innermost is in thermal contact with the LT and the sample chamber. The cooling of the sample is furthermore mediated by a small amount of Helium, serving as exchange gas inside the 
sample chamber at a pressure below 1 mbar. The space between the thermal shields is evacuated to $5 \times 10^{-6}$ mbar using a turbo pump (Pfeiffer Vacuum).

During experiments, the pressure inside the LT is reduced to around 20 mbar by pumping with a rotary vane pump (Sogevac SV40) to reach temperatures below $4 \mathrm{~K}$. Under these conditions, the helium in the LT becomes super-fluid and the temperature of the base plate inside the cryostat reaches $1.4 \mathrm{~K}$. Another temperature sensor, which is mounted on the sample insert and thus closer to the sample position (see Fig. 2.10 b), measures typically a value around $2 \mathrm{~K}$. This offset is probably caused by the larger cooling power at the base plate, which is in direct thermal contact to the LT, whereas the cooling of the sample insert is only mediated by the exchange gas.

In between measurements, the LT is kept at normal pressure and the exchange gas stays inside the sample chamber to ensure proper cooling of the naphthalene crystal. Using this storage procedure, DBATT molecules show no bleaching or any other photo-instability and thus, it is possible to work with the same DBATT molecule over months. Even after a temporary increase of the temperature to $60 \mathrm{~K}$, the DBATT molecules keep their resonance frequency.

Every once a while, the connection from UT to LT is obstructed, probably by frozen hydrogen or nitrogen. In these cases, the automated refilling is stopped and both tanks are repeatedly filled with warm helium gas and evacuated again until their connection is clear. During this process, the sample temperature rises by several ten degrees Kelvin but usually stays below the critical value of $60 \mathrm{~K}$.

The sample chamber consists of a copper base plate that is permanently inside the cryostat and a sample insert (see Fig. 2.10) that can be removed by using a load lock system without opening the cryostat. Two homebuilt piezo scanners, based on the slip stick principle [99], are mounted on the base plate. They are used to position the focusing and recollimation aspheric lenses (both A.W.I. Industries, NA $=0.55$ ) at the correct distance to the sample. A landing platform ensures correct alignment of the sample insert. Furthermore, there are two germanium and two Pt-10o temperature sensors on this plate. More details about the base plate and the transfer system can be found in [71].

A new sample insert was designed and constructed for the experiments performed in this thesis (Fig. 2.10 b). Three piezo sliders (Attocube) allow sample movement in every direction. The sample holder attached to the scanners is made of $1 \mathrm{~mm}$ thick aluminum. The sample itself sits in a $10 \mathrm{x}$ $10 \mathrm{~mm}$ cutout and is held in place with a thin aluminum band. 

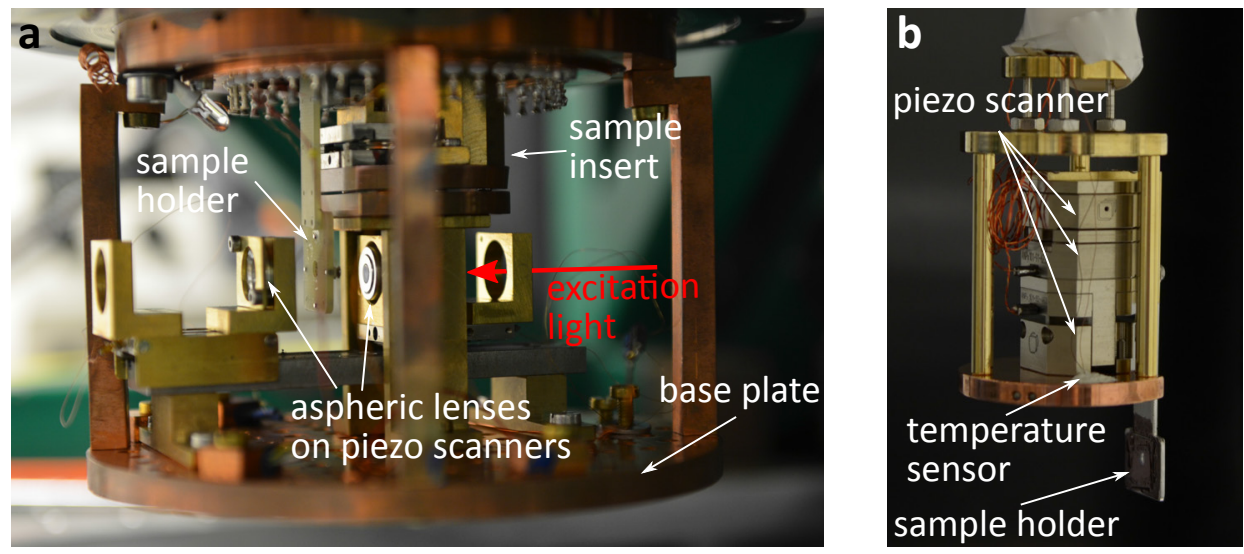

Figure 2.10: a, Picture of the base plate inside the cryostat with two aspheric lenses mounted on piezo scanners and the sample insert on its landing platform. Note that the sample insert shown here is the old version before the change to commercial piezo scanners. b, Picture of the new sample insert with three piezo scanners.

Placing the focusing optics inside the cryostat minimizes the distance between aspheric lens and sample, thus maximizing the possible NA. However, most commercial microscope objectives severely degrade at low temperatures, due to different thermal expansion of their elements. To circumvent this, we focus the light with a single aspheric lens. This comes with the drawback of various aberrations associated with a single aspheric lens. Most notable is the chromatic aberration, leading to different focal lengths for light at the o-o ZPL $(619 \mathrm{~nm})$, and the red-shifted fluorescence. Hence, imaging a molecule on the CCD camera results in a point spread function noticeably larger than the diffraction limit.

To further increase the NA of the focusing optics, a solid immersion lens (SIL) (A.W.I. Industries) [102] made of cubic zirconia is attached directly to the sample. The high refractive index of cubic zirconia (2.15 at a wavelength of $619 \mathrm{~nm}$ [103]) decreases the focus diameter and magnifies the image. However, the SIL introduces aberrations for objects that are not precisely on the optical axis, which become more severe with increasing distance. This limits the field of view to $\pm 20 \mu \mathrm{m}$ around the center. Furthermore, the SIL enables a more efficient collection of the light scattered by the molecule by preventing total internal reflection at the naphthalene interface and changing the emission pattern of the DBATT molecules such that they dominantly emit into the SIL (see Fig. 2.11). A more detailed discussion follows in section 3.3.

Most SILs deviate from the perfect hemispherical shape [96]. While interferometric measurements show that the mean radius of curvature is very 

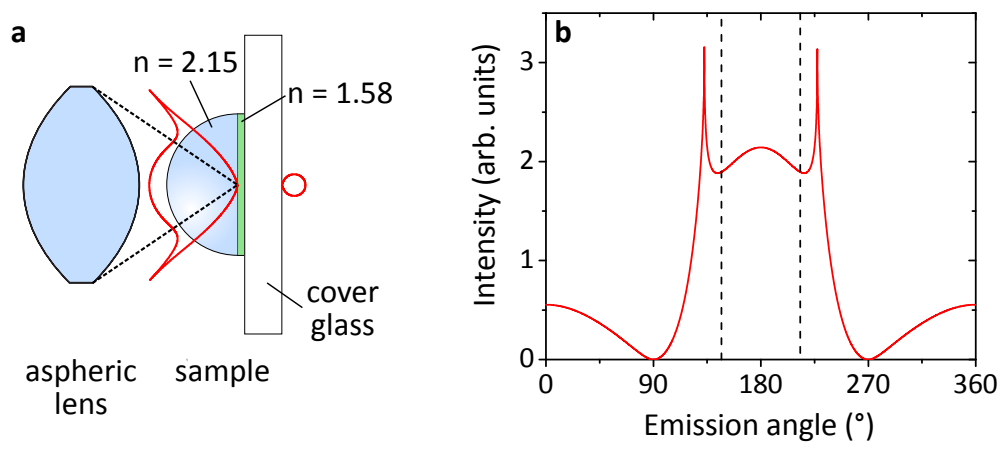

Figure 2.11: Emission pattern of a DBATT molecule at $50 \mathrm{~nm}$ distance to the SIL-naphthalene interface and with a dipole moment parallel to the interface. The black dashed line indicates the collection angle of the aspheric lens. a, Polar plot of the emission pattern. Here, the red line on the right hand side of the cover glass depicts the pattern in vacuum after propagation through the cover glass-vacuum interface. $\mathbf{b}$, Plot of the same emission pattern in a Cartesian coordinate system. Please note that the calculation of the emission pattern just takes the SIL-naphthalene interface into account. The propagation through the cover glass-vacuum interface is done using the Fresnel equations.

close to the specified $1.5 \mathrm{~mm}$, the curved surface typically deviates up to $200 \mathrm{~nm}$ from the ideal shape, giving rise to small aberrations (see Fig. 2.12). Furthermore, the flat side is usually around $30 \mu \mathrm{m}$ too far away from the curved side (labeled as $d$ in Fig. 2.13). Due to the small working distance of the aspheric lens of $1.6 \mathrm{~mm}$, this can result in aspheric lens and SIL touching each other before the flat side of the SIL, with the sample, enters the focus.

To avoid this problem, we further polish the flat side of the SILs until the thickness is correct to within a few micrometer. Afterwards, the SILs are characterized in a room temperature setup by imaging $200 \mathrm{~nm}$ fluorescent beads (Life Technologies) spin coated on the flat side of the SIL.

Inside the cryostat, the relative alignment of aspheric lens and SIL is crucial for achieving a diffraction-limited focus. First, coarse alignment is done by centering the SIL via visual inspection. Further fine adjustment is performed by monitoring the laser light reflected from the SIL. Two different reflections can be seen: one originates from the light reflected from the curved surface of the SIL (blue line in Fig. 2.13) and the other one from the light scattered off the flat side (red line in Fig. 2.13). For a perfect SIL, both reflections are focused onto the camera at the same time. However, the residual thickness $d$ of the bottom layer shifts one reflection out of focus. Hence, the position of the aspheric lens must be adjusted to switch from situation a, where the curved surface reflection is focused onto the camera, to situation $\mathbf{b}$, where the flat surface is in focus. Note that due to diffraction at the SIL air interface, the 

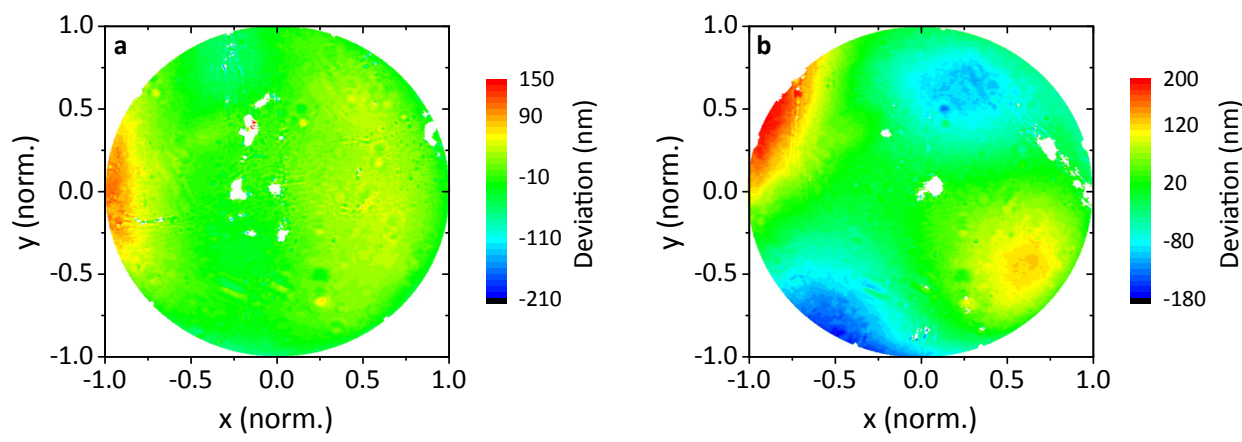

Figure 2.12: Interferometric measurement of the curved surface of two different SILs. The plot displays the deviation from the ideal hemispherical shape. The SIL in $\mathbf{b}$ shows a small astigmatism, while the SIL in a has no distinct aberration. Courtesy of Klaus Mantel.
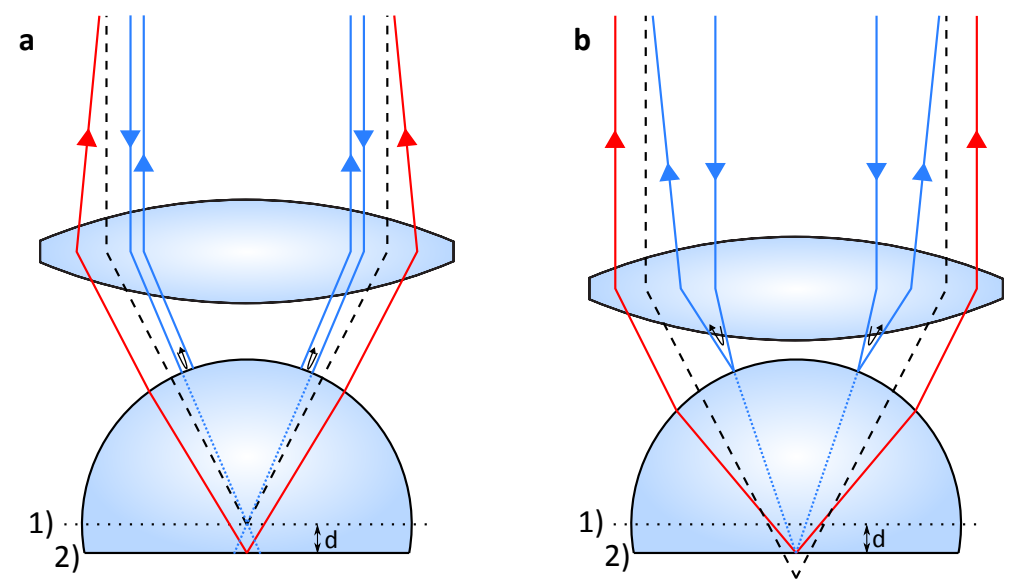

Figure 2.13: Schematic of the beam path through an imperfect SIL. The dotted horizontal line indicates the bottom of a perfect half-sphere (labeled as 1)), while the actual bottom of the SIL is labeled as 2) with $d$ being the offset between the two planes. The dashed black line shows the position of the focus of the asphere in absence of the SIL. The solid blue line shows the propagation of a beam reflected from the curved surface of the SIL, while the dotted blue line shows the propagation through the SIL. The red line represents the imaging of a spot at the bottom of the SIL. a, The focus of the aspheric lens is in plane $\mathbf{1}$ ). $\mathbf{b}$, The focus of the aspheric lens is adjusted such that the laser light is focused into plane 2). 


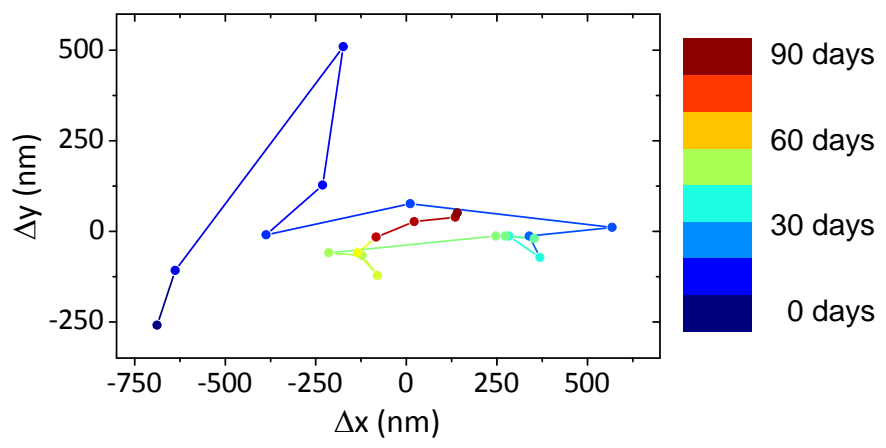

Figure 2.14: Drift of a molecule over 9o days. The dots represent the measured positions and the lines are just a guide to the eye. Note that the measurements are not evenly distributed across the timespan. The color indicates the time that has elapsed since the first measurement.

necessary movement of the aspheric lens exceeds the thickness of the bottom layer. By superposing the reflections from the two sides of the sample, proper lateral alignment of the SIL can be ensured.

\subsubsection{Mechanical stability}

Vibrations and drift lead to a lateral displacement of the focal spot decreasing the light-molecule coupling efficiency. Thus, the mechanical stability of the setup is crucial for the measurements. We estimate the vibration amplitude by comparing the noise of the transmitted laser power when the beam is focused on a flat region of the naphthalene crystal and directly on a crystal defect. In the latter case, small position variations lead to a five-fold increase of the noise. We convert the noise signal to a spatial vibration amplitude by comparison to the change of transmission when raster-scanning the focal spot across the defect region. In this manner, we deduce a vibration amplitude of $5 \mathrm{~nm}$ within $100 \mathrm{~ms}$.

On the time scale of hours and more, drifts become dominant. Figure 2.14 shows the trace of one molecule over a timespan of 90 days. The maximum change in position is less than $1.5 \mu \mathrm{m}$. Even after 30 days, the molecule was found within $70 \mathrm{~nm}$ of its former position. As long as the temperature in the cryostat stays below $5 \mathrm{~K}$ typical drifts over 24 hours are in the range of $100 \mathrm{~nm}$. This small drift rate enables continuous measurements over several hours without re-adjusting the position of the focal spot.

It is difficult to determine the origin of drifts and vibrations. One possibility concerns the galvanometric mirrors used for laser scanning. A position change of $10 \mathrm{~nm}$ on the sample corresponds to an angle variation of $7 \mu \mathrm{rad}$, 
which could be explained by the typical jitter of a galvanometer with analog servo drive. Further vibration sources are the vacuum pumps connected to the isolation vacuum and the lower helium tank, respectively. Although these are placed $5 \mathrm{~m}$ away, their vibration might couple via the tubing to the cryostat causing a displacement of the sample. The long term drift could be caused by small temperature variations outside the cryostat, which affect the optical alignment and in particular the tilt of the galvanometric mirrors.

\subsubsection{Sample preparation}

The sample consists of a thin naphthalene crystal doped with DBATT and enclosed between a SIL and a fused silica cover glass. The DBATT/naphthalene mixture is prepared by adding a small grain of DBATT (Institut für PAH-Forschung) to several $100 \mathrm{mg}$ of high purity naphthalene (Sigma Aldrich). The balances available in our laboratories are not able to reliably weigh amounts of less than $100 \mu \mathrm{g}$, hence no concentration can be given. The mixture is heated to $80^{\circ} \mathrm{C}$ to melt the naphthalene and shaken to ensure a homogeneous mixture. From this so-called stock solution, several different dilutions in the range of 1:10 to 1:100o are made and tested until the desired concentration of molecules is reached.

The first samples were prepared by putting a small flake of the DBATTnaphthalene mixture between a SIL and a cover glass. Subsequent melting of the naphthalene on a hot plate resulted in a thin, liquid film between SIL and cover glass. When the sample was removed from the hot plate, the naphthalene solidified within a few seconds. While this method is quick and easy to implement, it yields crystals of uncontrolled thickness, usually in the range of several $\mu \mathrm{m}$.

To have more control over the sample properties, a new technique was developed. Via optical lithography and subsequent reactive ion etching, a $500 \mu \mathrm{m}$ wide channel is prepared in a $500 \mu \mathrm{m}$ thick fused silica cover glass (see Fig. 2.15 a). The channel depth can be controlled via the etching parameters and was $220 \mathrm{~nm}$ for the experiments presented in the next chapter. A thoroughly cleaned SIL is placed directly on top of the channel. The positioning is done on an inverted microscope to ensure that the center of the SIL coincides with the middle of the channel. Smooth surfaces enable optical contact of SIL and cover glass, effectively joining the two by van-der-Waals forces [104]. In this way, the SIL creates a confined nano-channel for the crystal. The SIL is secured in its position with an aluminum band to prevent detachment during cool down. 
Subsequently, a millimeter-sized piece of a DBATT in naphthalene mixture is placed on the cover glass, next to the SIL and the whole sample is heated by a Peltier element until the naphthalene melts. The liquid naphthalene is drawn into the channel by capillary forces [105]. The inverted microscope enables monitoring of the filling process. Once the channel is filled, the Peltier element is switched off and the naphthalene solidifies within less than a minute. Sometimes this process has to be repeated several times until the center region is covered with a homogeneous crystal. Figure $2.15 \mathbf{b}$ shows a microscope image of a filled channel. The dark upper left corner shows the channel boarder which is not covered with the naphthalene crystal. The channel itself is covered with crystals of varying grain sizes. The DBATT molecules tend to accumulate at defects like grain boundaries. The DBATT molecules within one grain have mostly the same dipole orientation.

At ambient temperatures naphthalene has a high gas pressure, leading to a large evaporation rate. The confined geometry of the channel, reduces that problem. Nevertheless, we usually cool down the sample in the cryostat within a few hours after the preparation to prevent evaporation.

\subsection{Single molecule characterization measurements}

Several measurements are performed routinely on a single molecule to characterize its properties. They can be separated into spectral, spatial, and widefield measurements. The first two types are performed in the confocal mode: the excitation laser is focused to a single spot on the sample and the light emitted from that point is detected with a SPCM or a spectrometer. This technique allows the acquisition of spectral and spatial information about a single molecule. On the other hand, it is possible to investigate a larger region of the sample by using wide-field illumination. In that case, information about several molecules can be acquired at the same time by imaging the sample onto a sensitive CCD camera. However, this comes at the cost of longer integration times, and analysis of the coherent interaction of light and molecule is difficult in this configuration [96]. Hence, wide-field measurements are the first step of sample characterization, while more detailed measurements are taken in the confocal mode on a single molecules.

\subsubsection{Wide-field illumination}

Wide-field illumination in combination with detection on a CCD camera investigates a large area of the sample at the same time. Within a few hours, the 


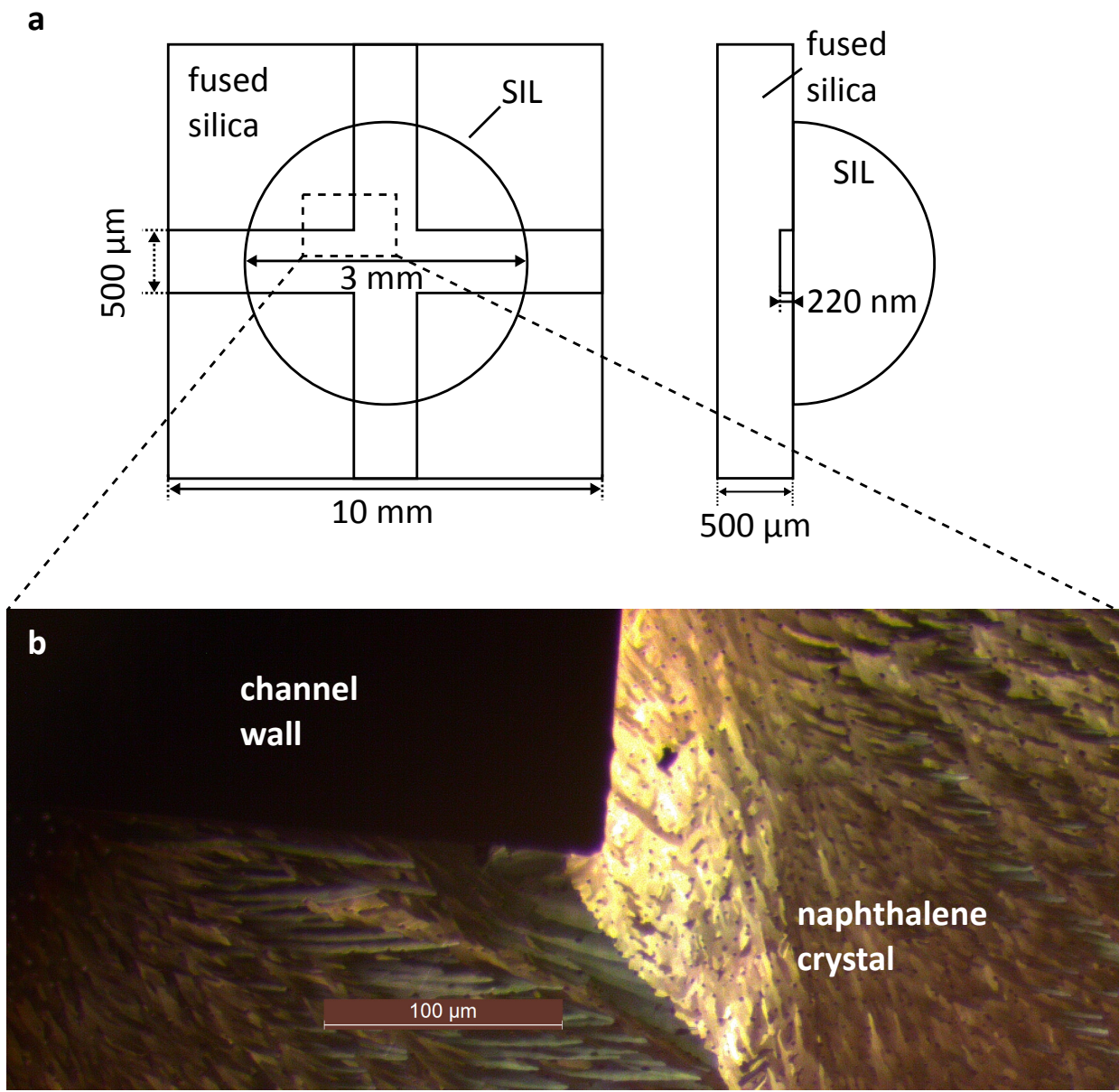

Figure 2.15: a, Drawing of a nano-channel inside a fused silica cover glass with attached solid immersion lens (SIL). b, Microscope image of a channel filled with DBATT in naphthalene. Recorded with transmitted light and cross polarization. See text for details. Courtesy of Benjamin Gmeiner.

basic information of hundreds of molecules can be obtained. From this list, a molecule at the desired position and frequency can be chosen. Especially for future experiments that might require two dipole-coupled molecules $[58,96$, $100]$, or molecules in the proximity of a nano-structure [80-83], this method becomes increasingly important.

The illumination is changed from confocal to wide-field by adding an additional lens in the excitation path. This lens focuses the laser beam into the back focal plane of the aspheric lens, which then collimates the beam (Fig. 2.16). The SIL focuses the beam again, but to a far smaller extent than 


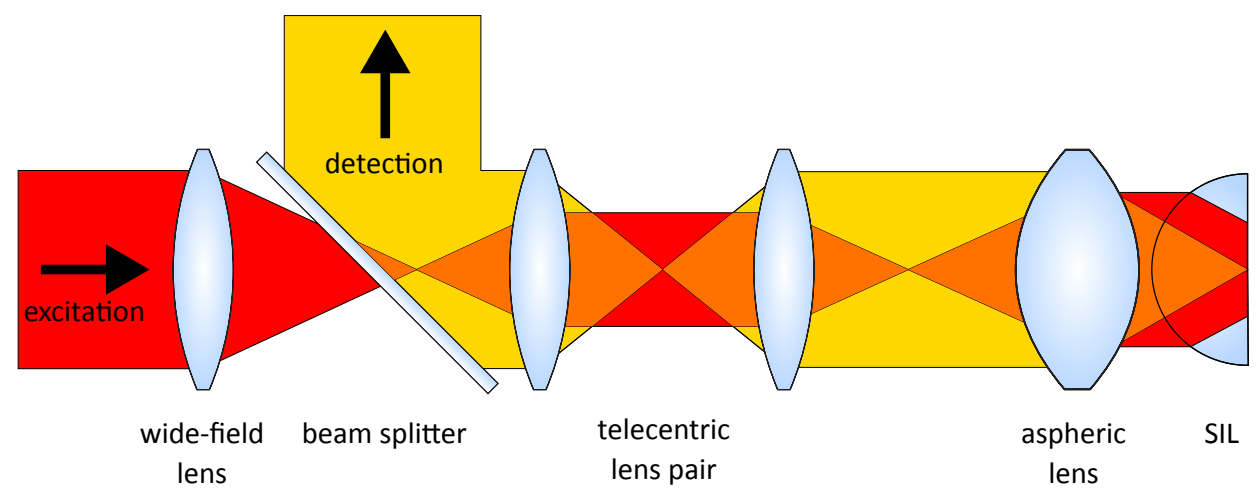

Figure 2.16: Propagation of the excitation beam (red) and the light coming from the sample (yellow) in the case of wide-field illumination.

without the wide-field lens. This leads to the illumination of a region with a $20 \mu \mathrm{m}$ diameter on the sample.

The emitted fluorescence light is imaged onto a CCD camera (Hamamatsu Orca R2). Scanning of the laser frequency and acquisition of camera images is synchronized by trigger pulses from the data acquisition card (National Instruments) which at the same time controls the laser scanning. The camera is operated in the "synchronous readout" mode, where the exposure of an image starts with one trigger pulse and stops with the next one, which at the same time starts the exposure of a new image. The typical integration time for one frame is $100 \mathrm{~ms}$ and the excitation frequency is changed in steps of $10 \mathrm{MHz}$.

A laser frequency scan results in a video of several thousand camera frames, where one can identify different molecules. The synchronization with the laser scan allows the assignment of an excitation frequency to each frame. From the evaluation of these videos, linewidth, transition frequency, and position of all excited molecules can be obtained.

The evaluation is done with a Matlab script which is sketched in Fig. 2.17 and described in the following. First, a background is subtracted from each frame and the positions of all local maxima with a signal-to-noise ratio (SNR) above a certain threshold (typically $2-5$ ) is recorded. The center of these spots is determined by fitting a two-dimensional Gaussian profile. This results in a preliminary list of positions and frequencies of molecules.

Since a molecule typically appears in several frequency frames, these events are connected in the next step. For each element in the preliminary list, a fluorescence excitation spectrum covering a range of $\pm 500 \mathrm{MHz}$ is obtained by combining the data of several frames before and after the initial frame. 


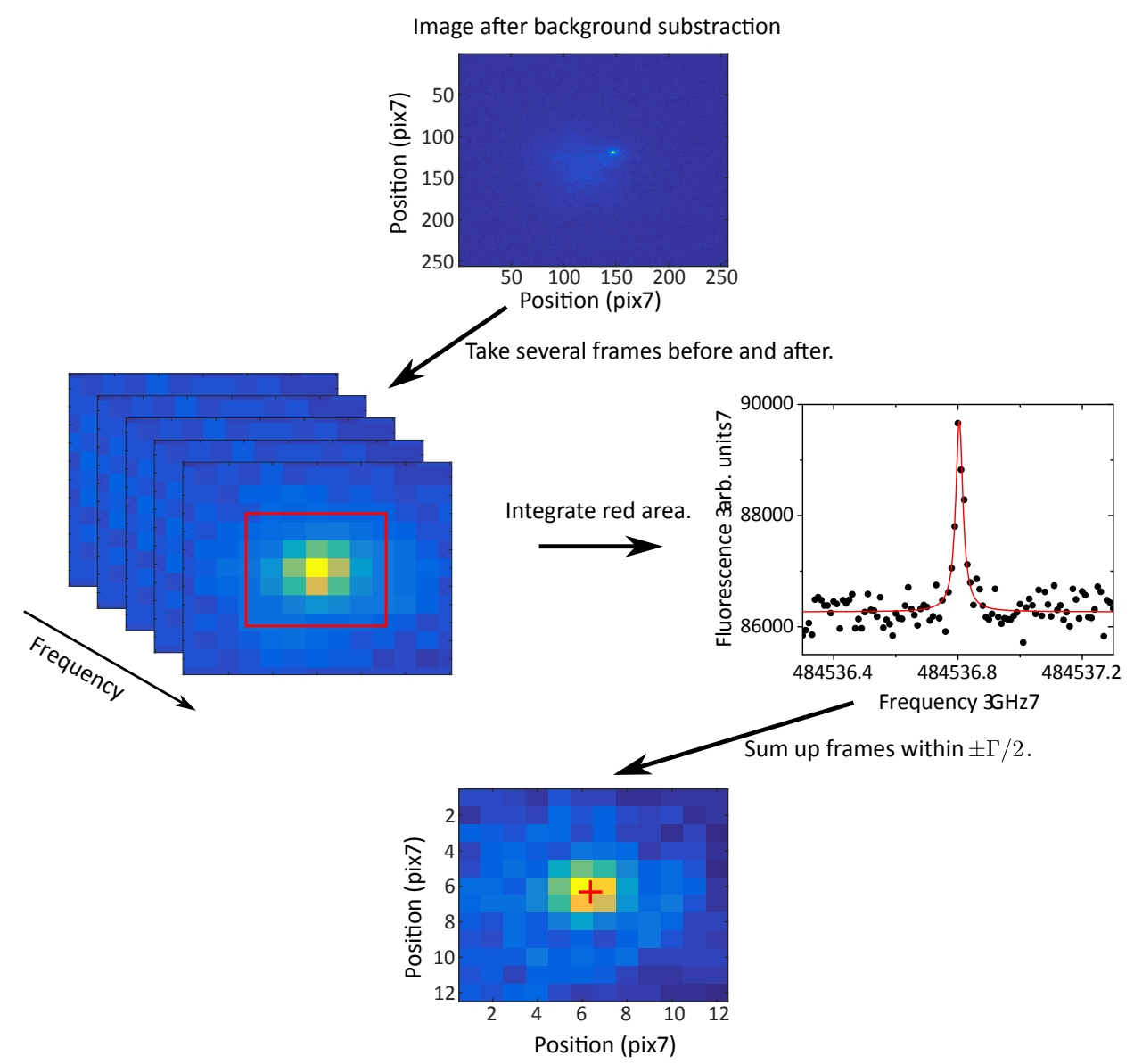

Figure 2.17: Schematics of the steps within the localization algorithm: A local maximum is found in a frame after background subtraction. By integrating the region around this peak for several frames, a fluorescence excitation spectrum is obtained. A fit to that spectrum yields the center frequency and the linewidth of the molecule. Finally, a sum image is generated by combining all frames within half a linewidth of the center frequency. From that image, the position of the molecule is deduced by fitting a two dimensional Gaussian.

In each of these frames an area of \pm 3 times the standard deviation of the two-dimensional Gaussian fit is integrated. A Lorentzian is fitted to the resulting spectrum. Finally, all frames recorded at frequencies within half a linewidth of the molecular resonance are summed up to get an image of the molecule with a better SNR. Another two-dimensional Gaussian is fitted to the sum image and the resulting information, including the position, center frequency, and linewidth, is stored. Moreover, an "exclusion cube" is defined: 


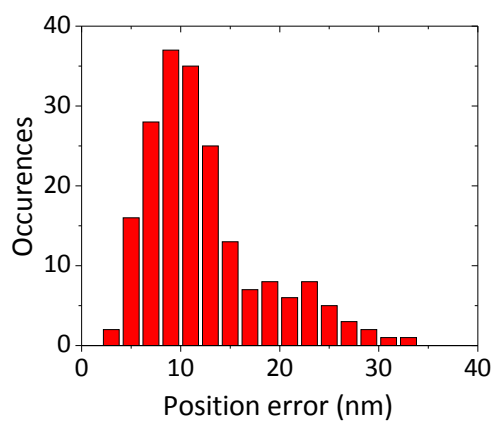

Figure 2.18: Distribution of the localization precision for 200 molecules.

All further events on the preliminary list that are spatially within three standard deviations and spectrally within half a linewidth of this molecule are assumed to belong to the same molecule and thus neglected to avoid multiple occurrences. After this, a manual post selection step might be necessary to remove molecules that showed strong blinking or fast spectral diffusion and thus appeared at several disconnected frequencies.

Each position on the CCD chip can be mapped to a tilt of the galvanometric scanning mirrors that is required to address the corresponding location on the sample in confocal mode. The tilt obtained by this method usually places the focus within $200 \mathrm{~nm}$ of the desired spot, which is sufficient to quickly center the focus on the respective molecule. The error results mainly from an imperfect calibration of the coordinate transformation from the CCD chip to the scanning mirror tilt. The localization precision is much higher.

The experiment operates in a regime where error contributions due to pixel size and background are negligible. In that case, the localization accuracy can be estimated by $\sigma \approx \sigma_{\text {Gauss }} / \sqrt{N}$, where $\sigma_{\text {Gauss }}^{2}$ is the variance of the Gaussian fit and $N$ is the number of detected photons [96]. As DBATT molecules do not bleach at low temperatures, in principle localization to an arbitrary precision should be possible by integrating for a long time. In practice, the precision is limited by the mechanical stability of the setup. As has been discussed in subsection 2.2.3, the drift is typically in the range of $100 \mathrm{~nm}$ over 24 hours. Figure 2.18 shows the standard deviation on the position for 200 molecules using the covariance matrix of the Gaussian fit. It ranges from $4-30 \mathrm{~nm}$ with a mean value of $12 \mathrm{~nm}$. 

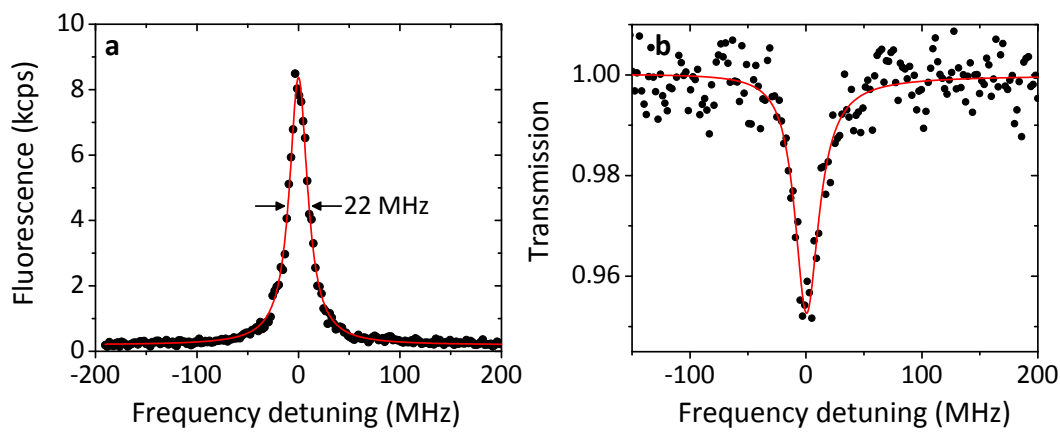

Figure 2.19: a, Fluorescence excitation measurement. b, Extinction measurement on a single molecule. The red line is a fit to the data.

\subsubsection{Spectral characterization}

By scanning either the frequency of the excitation laser or by analyzing the fluorescence light with a spectrometer spectral information about the molecule can be obtained. In a fluorescence excitation measurement, the laser frequency is swept across the resonance of a molecule and the emitted red-shifted fluorescence light is detected with a SPCM as shown in Fig. 2.19 a. By fitting a Lorentzian function (Eq. (2.27)) to the result, one can determine the linewidth and the center frequency. If furthermore several scans at different excitation powers are performed, a saturation curve can be extracted from the peak count rates and the broadening of the linewidth (see Fig. 2.5). The deduced saturation power is a figure of merit for the coupling efficiency as will be described in more detail in chapter 3 .

At the same time, the extinction signal (Fig. 2.19 b) can be measured in the transmission channel. In that case, a narrow band-pass filter is used to block any red-shifted light that is collected in the transmission path. As explained in subsection 2.1.6, the interference of the transmitted laser light and light scattered from the molecule leads to a drop in the transmitted power. Depending on the detection solid angle, the exact position of the focus, and the polarization of the laser beam, the shape can vary from Lorentz-shaped dips and dispersive signals to a peaked spectral feature $[60,71,106]$.

\subsubsection{Spatial characterization}

For a spatial measurement, the position of the focus is scanned across the sample by tilting the galvanometric mirrors, while the excitation frequency is kept at a fixed value - typically the resonance frequency of a molecule. The detected signal can be either the emitted fluorescence or the transmitted power. 

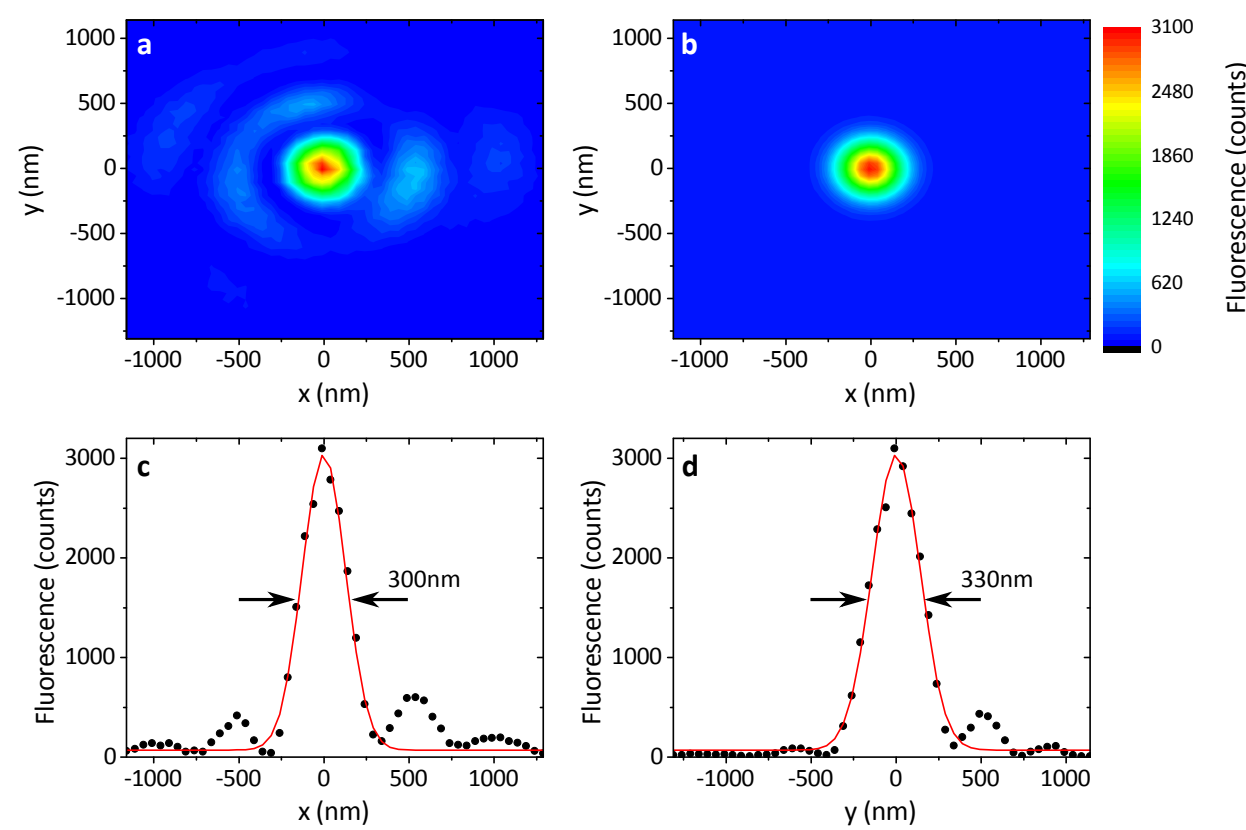

Figure 2.20: Laser raster scan of a single molecule. a, Measured $2 \mathrm{~d}$ fluorescence map as the focal spot is scanned across the position of the molecule. $\mathbf{b}, 2 \mathrm{~d}$ Gaussian fit to the measurement in a. $\mathbf{c}$ and $\mathbf{d}$ show line cuts in $\mathrm{x}$ and $\mathrm{y}$ direction respectively, where the solid red line is Gaussian fit. The FWHM of the focal spot is $300 \mathrm{~nm}$ in $x$ and $330 \mathrm{~nm}$ in $y$ direction.

The response of the molecule depends on the Rabi frequency $\Omega$, which is proportional to the scalar product of the dipole moment and the electric field at the position of the molecule. Taking advantage of this fact, we can use the molecule as polarization sensitive, point-like probe to sample the electric field of the laser beam.

In the limit of low excitation power, the rate of emitted fluorescence photons follows $R \propto \Omega^{2}$ (cf. Eq. (2.27) in the limit $S \ll 1$ ). In this regime, the detected fluorescence signal is proportional to the intensity of the laser field. Thus, an intensity map of the excitation beam can be obtained by raster scanning it across the position of the molecule. From this map, one can deduce and optimize the quality of the focus and precisely determine the position of the molecule on the sample. Figure 2.20 shows such a scan.

When a beam is tightly focused using high-NA optics, the polarization in the focus is no longer equal to the polarization state of the input beam. A beam that is, e.g., linearly polarized in $x$-direction, has also $y$ - and $z$-polarized components in the focus [107] (see Fig. 2.21 a for the choice of the coordinate 
system). These different components coexist on a small length scale of typically less than $500 \mathrm{~nm}$. Thus, a nanoscopic probe is necessary to reconstruct the polarization distribution [108]. A single molecule can serve as such a probe, as it is sensitive to the polarization of the incident light. If the polarization is rotated, e.g., with a half-wave plate, one can obtain a polarizationresolved intensity map of the focus. However, acquisition of quantitative information would require precise knowledge of the orientation of the dipole moment, which is currently not available. Yet, this information is in principle accessible, e.g., by back focal plane or defocused imaging [109].

Figure 2.21 $\mathbf{b}$ and $\mathbf{c}$ show a laser raster scan with two different polarizations of the excitation light. In $\mathbf{b}$, the laser beam is linearly polarized parallel to the projection of the molecular dipole moment on the $x$ - $y$-plane, which yields an intensity distribution similar to Fig. 2.20. For scan c, the input polarization is rotated by $90^{\circ}$. Hence, the dominant polarization component is perpendicular to the dipole moment and cannot excite the molecule. Thus, weaker components that are caused by the tight focusing become visible. The next strongest component is polarized in $z$-direction.

Parts $\mathbf{d}$ and e depict the expected intensity distribution if a Gaussian beam with a wavelength of $619 \mathrm{~nm}$, polarized in $x$-direction is focused using a lens with an NA of 0.55 , like the aspheric lens inside the cryostat. The $x$-polarized component $\left|E_{x}\right|^{2}(\mathbf{d})$ reproduces the circular shape of the measurement shown in $\mathbf{b}$. As the theoretical calculation does not include the SIL, the spot size of the theory plot is roughly twice as large. The intensity distribution of the $z$-polarized part $\left|E_{z}\right|^{2}$ (e) shows the same double-lobe structure that we see in c. This demonstrates that we indeed observe excitation via a new polarization component in the focus. If the input polarization had a significant degree of ellipticity, we would instead again measure a circular shape like in $\mathbf{b}$. The two lobes in $\mathbf{c}$ are rotated around the origin, because the polarization of the incident beam is not parallel to the $x$-axis. No traces of the $y$-polarized component are visible, because its intensity is 100 weaker than the $z$-component. The intensity of the theory plots is normalized to the peak intensity of $\left|E_{x}\right|^{2}$.

By comparing the measurement with the theoretical expectations, we can estimate the out-of-plane angle $\theta$ of the molecular dipole moment $\mathbf{d}$ [110]. After correction for background counts, the observed peak fluorescence is 13.7 times higher if the incident beam polarization is parallel to the projection of the dipole moment on the $x-y$-plane. The expected intensity of the $x$-polarized component is 23 times higher than the peak value of $\left|E_{z}\right|^{2}$. As the measurements are taken in the weak excitation limit, the fluorescence 


\section{Single molecule optics}

a
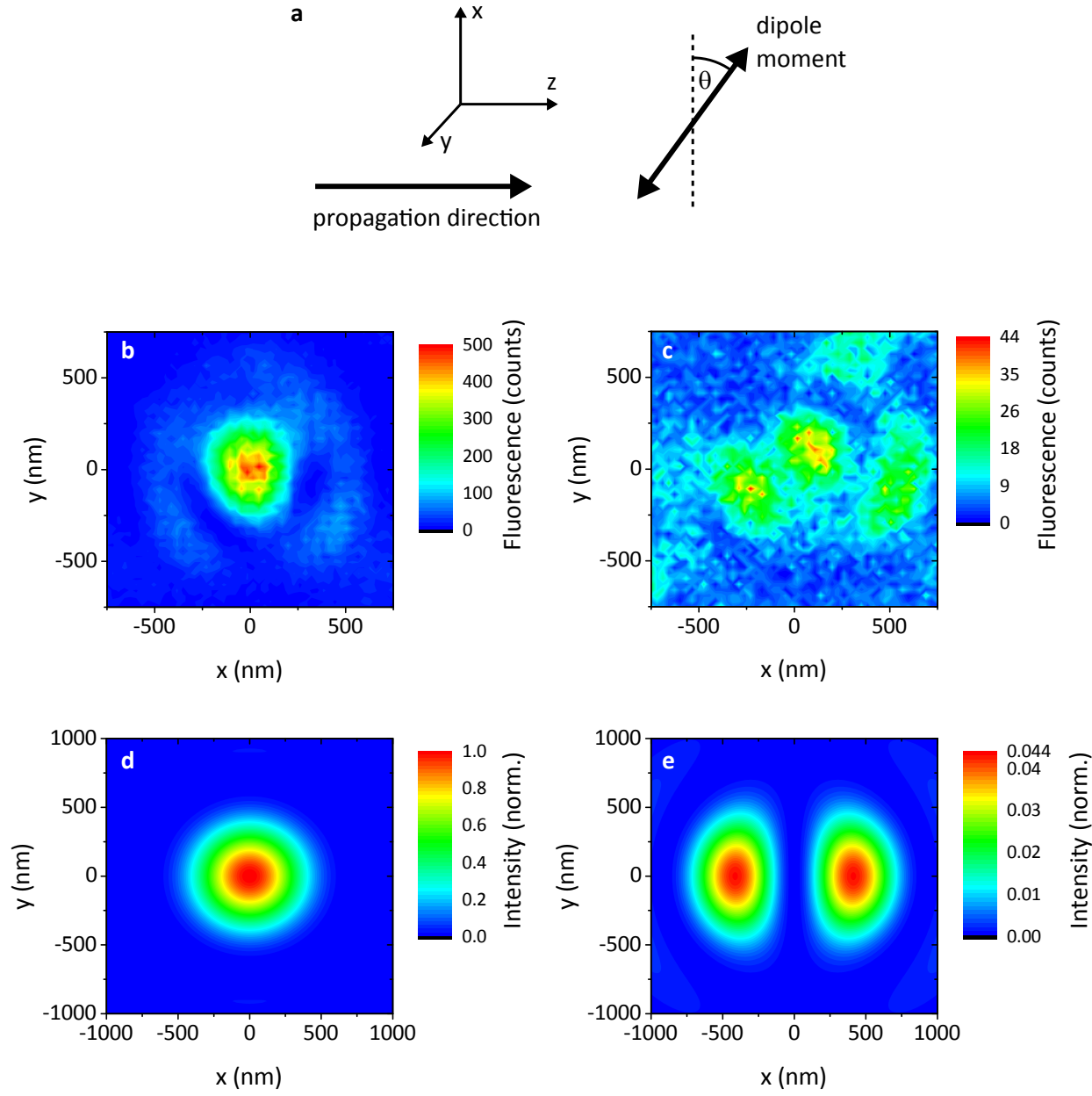

Figure 2.21: a, Coordinate system: $z$ is the beam-propagation direction and the tilt of the molecular dipole moment out of the $x-y$-plane is labeled $\theta$. $\mathbf{b}$ and $\mathbf{c}$, Laser raster scan of single molecule with the polarization of the excitation beam oriented parallel to the molecular dipole moment (b), and rotated by $90^{\circ}(\mathbf{c})$. Note that the color bars differ by a factor of 10 for $\mathbf{b}$ and c. $\mathbf{d}$ and $\mathbf{e}$, Calculated intensity map of the polarization component in $x$ - (d) and $z$-direction (e) for a Gaussian input beam with polarization in $x$-direction. Theory plots by courtesy of Peter Banzer. 
count rate is proportional to $|\mathbf{E d}|^{2}$. Thus, we can write

$$
\begin{gathered}
d_{x}^{2}\left|E_{x}\right|^{2}=13.7 d_{z}^{2}\left|E_{z}\right|^{2} \\
\left|E_{x}\right|^{2}=23\left|E_{z}\right|^{2} \\
\Rightarrow d_{z}=1.3 d_{x},
\end{gathered}
$$

where $d_{x}$ and $d_{z}$ are the dipole moment components in $x$ - and $z$-direction. The relation of $d_{x}$ and $d_{z}$ given in Eq. (2.45) corresponds to an angle of $\theta \approx$ $52^{\circ}$.

This estimate is a strong indication for a significant rotation of the molecular dipole moment out of the $x-y$ (sample) plane. Unfortunately, similar measurements were not performed with other molecules, thus we cannot conclude anything about the general out-of-plane orientation of DBATT molecules in a naphthalene matrix. However, this method is an interesting option for the routine determination of $\theta$, as no changes to the experimental setup are necessary, in contrast, e.g., to back focal plane imaging. For a more precise estimation of $\theta$, the field distribution in the focus should be calculated taking the SIL into account or directly measured with a nanoscopic probe [108].

\subsection{Molecule pairs}

Having established control of a single molecule, one can start to build up small ensembles of several emitters. The first step along that line is the investigation of molecule pairs. If two emitters have a spacing of less than a transition wavelength, they can interact via dipole-dipole coupling. The pair then forms a coupled system, with new eigenstates (see Fig. $2.22 \mathrm{a}$ ), which are coherent superpositions of ground and excited states of the individual emitters. The new eigenstates are [10o]:

$$
\begin{aligned}
|U\rangle & =|e e\rangle, \\
|J\rangle & =\sin \phi|e g\rangle+\cos \phi|g e\rangle, \\
|I\rangle & =\cos \phi|e g\rangle-\sin \phi|g e\rangle, \\
|G\rangle & =|g g\rangle,
\end{aligned}
$$

where the mixing angle $\phi$ depends on the coupling strength and the relative detuning of the two emitters. When recording a fluorescence excitation spectrum of such a coupled system, one of the interesting features is the appearance of a third peak in between the transition from $|G\rangle \rightarrow|I\rangle$ and $|G\rangle \rightarrow|J\rangle$. 

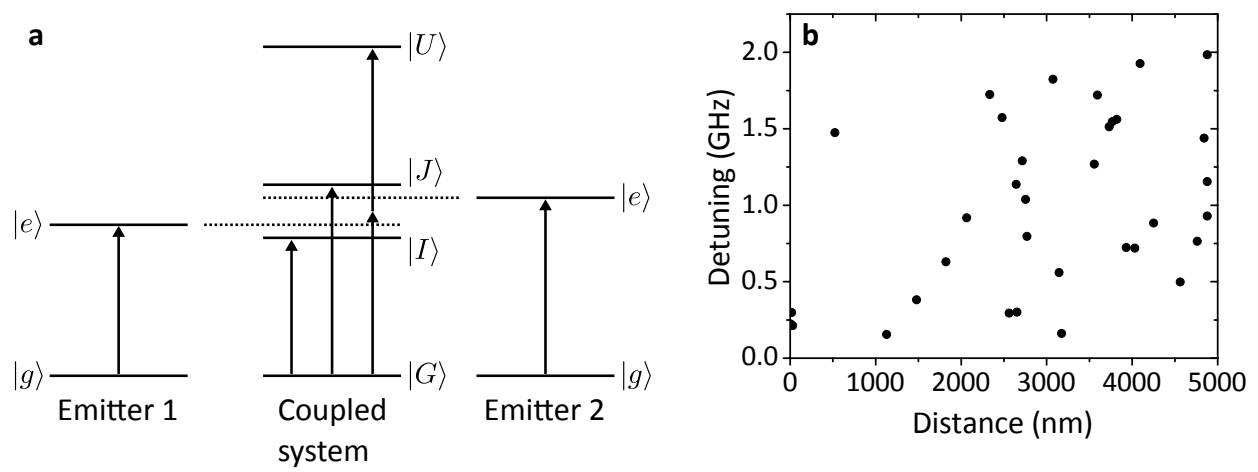

Figure 2.22: a, Level scheme of two dipole-dipole coupled emitters. The arrows indicate the possible transitions. $\mathbf{b}$, Spatio-spectral molecule pair map. Each point indicates the distance and relative detuning of a molecule pair.

It is caused by a two-photon excitation, which transfers the system directly from the ground state $|G\rangle$ into the doubly excited state $|U\rangle$. This transition has a quadratic intensity dependence and was indeed observed for two coupled terrylene molecules [58].

However, it is very unlikely that two molecules are close enough in space and transition frequency that they show significant dipole-dipole coupling. Thus, a large number of molecules has to be checked to maximize the chances of finding such a pair. To that end, we use wide-field illumination combined with CCD camera detection, as described in subsection 2.3.1.

From that data, we generated maps that show molecule pairs as a function of their spectral and spatial distance (see Fig. 2.22 b). We then investigated the pairs with a distance of less than $200 \mathrm{~nm}$ and a frequency difference below $5 \mathrm{GHz}$ closer in confocal mode. In particular, we were looking for the emergence of the two-photon transition peak. Even though we investigated more than 2800 molecules, giving rise to 45 potential pairs, we could not identify dipole-coupled molecule pairs. Often the "pair" turned out to be just a single molecule undergoing frequency jumps, which thus appeared at two different frequencies. In the few cases, where the pair was actually formed by two stable molecules, we could not see any sign of the two-photon peak. As we can only determine the distance in the sample plane, it is possible that the two molecules were sitting at different depths inside the nano-channel.

The obvious way of improving the likelihood for close molecule pairs is increasing the concentration of DBATT inside naphthalene or reducing the channel depth. Another approach could be the use of chemically linked molecule pairs [111], where the covalent bond ensures that the molecules are 

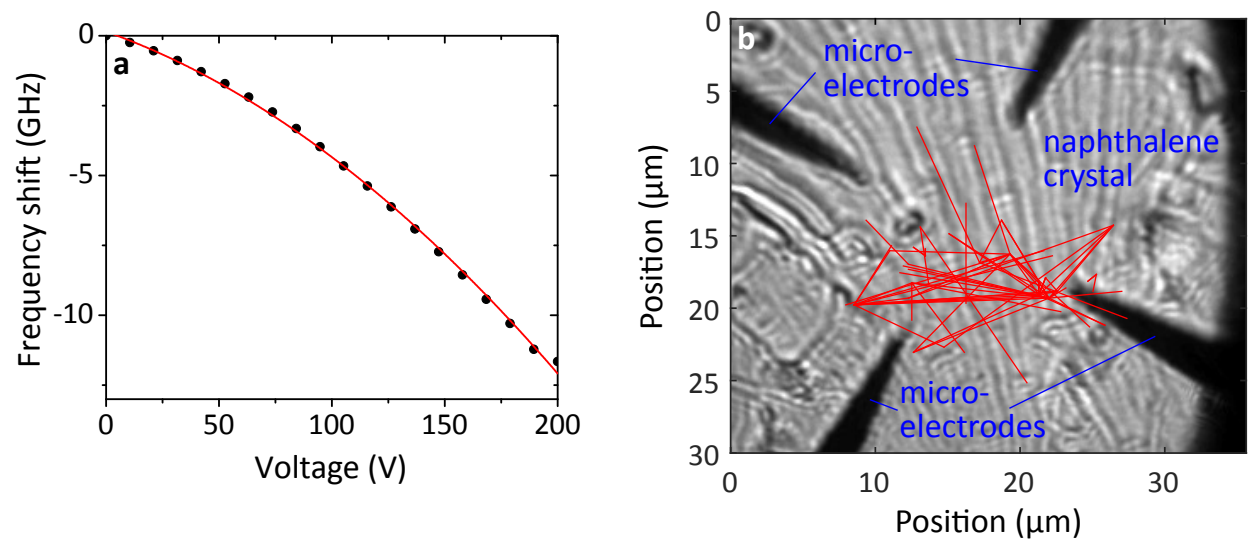

Figure 2.23: a, DC-Stark shift of a molecular resonance. The solid red line is a second order polynomial fit. b, Image of a sample with micro electrodes. The lines connect molecules that can be brought into resonance, by adjusting the tuning voltages.

close to each other. Although the bond could have a negative impact on the photo-physical properties.

But also molecule pairs not showing dipole-dipole coupling could yield interesting effects. The emission of two molecules within one diffractionlimited volume is directed into the same spatial mode and can show interference if they are driven coherently. For large ensembles of emitters with an inhomogeneous frequency distribution, this interference reduces the emission decay time, a phenomenon called optical free induction decay [89, 112]. If instead just a few emitters are present, we could observe a beating of the single photon emission occurring when the driving field is switched off.

For these investigations, the ability to tune molecule pairs into and out of resonance is important, as it can be used to control the coupling strength, in the case of dipole-dipole coupled molecules, and the beating frequency, in case of free induction decay. One way of achieving this, is the application of static electric fields to shift the transition frequency via the DC-Stark effect [113]. To alter the relative detuning of two molecules, we require different electric fields at their respective positions. For this reason, we structured gold electrodes with sharp tips (see Fig. 2.23 b) inside the nano-channel, which generate a highly inhomogeneous electric field distribution.

Figure 2.23 a shows the resonance frequency shift of a single DBATT molecule as a function of the voltage applied to one of the electrodes, while the other three are grounded. A maximum shift of $-12 \mathrm{GHz}$ is achieved at $200 \mathrm{~V}$. The observed Stark shift depends linearly and quadratically on the 
voltage, with both components being of almost of equal strength at the highest applied voltage. In contrast to our findings, Ref. [113] reports an almost exclusively quadratic Stark shift for DBATT in naphthalene. This discrepancy could be caused by the confined geometry of the crystal inside the nanochannel, leading to a non-inversion-symmetric insertion site for DBATT in the naphthalene crystal, which would in turn result in a linear Stark shift component.

To investigate the tuning ability, we recorded the Stark shift of many molecules in parallel, using wide-field illumination. In Fig. 2.23 b, molecules whose resonance frequency cross in the available voltage range are connected by red lines. In particular, there are many occurrences of molecules separated by several micrometers that can be tuned into resonance by applying a voltage to the gold electrodes. Furthermore, we observed a relative shift of $90 \mathrm{MHz}$ of two molecules that were only separated by $300 \mathrm{~nm}$, providing evidence that the electrodes generate fields with significant inhomogeneities on a sub-micrometer scale. 


\section{Photon-molecule coupling efficiency}

When light passes a single emitter usually only a small fraction of the incident photons are scattered, even if the beam is resonant to the emitter. However, the observation of coherent light-matter interaction, e.g., by measuring the transmitted power (see subsection 2.1.6), requires efficient coupling as only then the signal changes significantly. For this purpose, one approach is placing the emitter inside a high-finesse optical cavity. Trapped between two high-reflectivity mirrors, the photons pass the emitter several hundredthousand times thus compensating the low single-pass interaction probability [114]. Yet, the experimental realization of such a cavity is very challenging and hence several experiments, including this work, try to achieve large coupling efficiencies by just using tight focusing [60, 115-117].

The evident question of how efficiently focused light can interact with a single emitter is addressed in this chapter. We begin by discussing the maximum possible response of a TLS to an external driving field of given strength. The result is that a minimum incident rate of a quarter photon per lifetime ${ }^{1}$ is required to excite the emitter with a saturation parameter of $S=1$. In the second section, we compare this benchmark with experimentally measured values, and investigate the relation of extinction signal and coupling efficiency. In the last section, we consider possible approaches to increasing the coupling efficiency. Furthermore, we present first results in this direction from experiments with a gallium phosphide SIL and gold micro-mirrors.

\subsection{Definition}

The coupling efficiency connects the incident power, or equivalently the incident photon rate, to the response of the molecule. By comparing the experimentally achieved value to the theoretically possible maximum, we define a coupling efficiency.

The response of the molecule to an electric field can be described by the Rabi frequency or the saturation parameter, which in turn are functions of

${ }^{1}$ As explained later on, this is valid for focusing from a $2 \pi$ solid angle. In case of focusing from the full $4 \pi$ solid angle, the necessary photon rate drops to $1 / 8$ photon per lifetime. 
a

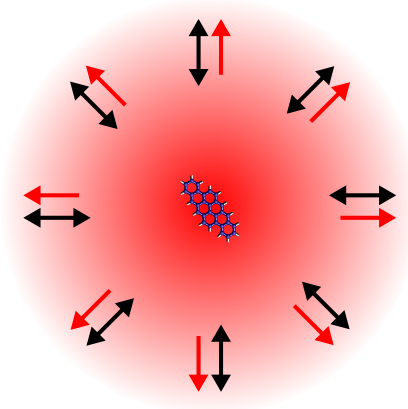

b

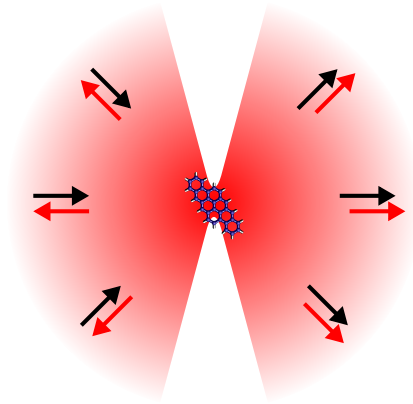

Figure 3.1: a, Focusing light from the full $4 \pi$ solid angle onto a TLS. The incident light (black arrows) and the scattered light (red arrows) interfere in every direction. b, Focusing light from a solid angle of less than $2 \pi$ onto a TLS. Interference of incident light and scattered light happens only in forward direction.

the electric field amplitude $E_{\text {inc }}(\mathcal{O})$ at the position of the molecule. For simplicity, we assume that $\mathbf{E}_{\text {inc }}(\mathcal{O})$ is parallel to the molecular dipole moment $\mathbf{d}$. The incident power $P_{\text {inc }}$ that is necessary to achieve a certain $E_{\text {inc }}(\mathcal{O})$ depends on the spatial mode of the input field and the focusing optics. It is intuitive that the incident light drives the molecule most efficiently if $E_{\text {inc }}(\mathcal{O})$ is maximized for a fixed $P_{\text {inc }}$. The relation of $P_{\text {inc }}$ and $E_{\text {inc }}(\mathcal{O})$ can be described by the normalized energy density $F$,

$$
F=\frac{\epsilon_{0} E_{\text {inc }}^{2}(\mathcal{O})}{4 P_{\text {inc }}}=\frac{\epsilon_{0} \Omega^{2} \hbar^{2}}{4 P_{\text {inc }}|\mathbf{d}|^{2}},
$$

which has been calculated for several different field distributions [118].

An arbitrary input field can be described in terms of a multipole expansion around the position $\mathcal{O}$ of the molecule. From that expansion, only the dipole terms give a non-zero electric field at $\mathcal{O}$. Thus, $F$ reaches its maximum value if the incident field is completely composed of an electric dipole field. As a dipole radiates into every direction, this requires the incident field to come from a full $4 \pi$ solid angle (see Fig. 3.1 a). In that case, the normalized energy density is given by [119]:

$$
F_{\max }=\frac{k^{2}}{3 \pi c} \text {. }
$$

An alternative understanding of $F$ can be gained by looking at the scattering efficiency, i.e., the ratio of the scattered and incident power [66]

$$
\mathcal{K}=P_{\text {scat }} / P_{\text {inc }},
$$


which is related to $F$. The power scattered by a TLS is given by

$$
P_{\text {scat }}=\frac{1}{2} \hbar \omega_{0} \Gamma_{1} \frac{S}{S+1} \approx \hbar \omega_{0} \frac{\Omega^{2}}{\Gamma_{1}},
$$

where the approximation is valid in the weak excitation limit, i.e., $S \ll 1$ and for resonant excitation. With Eqs. (3.1) and (3.4), we can express $\mathcal{K}$ in terms of $F$ :

$$
\mathcal{K}=4 \frac{\omega_{0}|\mathbf{d}|^{2}}{\Gamma_{1} \epsilon_{0} \hbar} F .
$$

By inserting $F_{\max }$ in Eq. (3.5) and using Eq. (2.35), we can calculate the maximum possible value for $\mathcal{K}$, which yields $\mathcal{K}_{\text {max }}=4$ and agrees with the rigorous calculation presented in Ref. [97]. At first glance, this result seems to violate energy conservation, as it indicates that the TLS scatters four times the incident power. However, this maximum value is only achieved for an incident field coming from a solid angle of $4 \pi$, which means that scattered and incident fields interfere in every direction (see Fig. 3.1 a). A detector integrating a solid angle of $4 \pi$ would measure an outgoing power equal to $P_{\text {inc }}$, in agreement with energy conservation [97].

In practice, only few experimental approaches aim at exciting a TLS with a wave coming from (almost) the full $4 \pi$ solid angle [117]. The more common approach is to focus a light field from a solid angle of $2 \pi$ or less (see Fig. 3.1 b). In that case, a maximum value of $\mathcal{K}=2$ is achieved for the excitation with a directional dipole wave $[66,97]$. As before, the scattered power is larger than the incident power, seemingly violating the law of energy conservation. But again, interference solves this contradiction: half of the scattered power propagates in the same direction as the incident beam and both fields interfere destructively as their relative phase difference is equal to $\pi$. Hence, no power is detected in forward direction corresponding to a perfect extinction measurement, as described in subsection 2.1.6, with an efficiency parameter $\eta=1$. The other half of the scattered light is going backwards leading effectively to the reflection of the incoming beam by a single emitter [66].

In principle, we could employ $\mathcal{K}$ as figure of merit to quantify the photonmolecule coupling efficiency. But the value of $\mathcal{K}$ depends on the absolute incident power as the emission rate of a TLS saturates with increasing excitation. Hence, even though the focusing of the incident beam is still optimal, the scattering efficiency might not achieve the maximum value. One way to circumvent this behavior would be to specify the scattering efficiency always in the weak excitation regime. We use a different approach and define a coupling efficiency $\xi$ which is independent of the absolute excitation. To that 
end, we compare the incident power required to excite the molecule with a given saturation parameter $S$ in the ideal case, $P_{\text {ideal }}(S)$, with the experimentally determined value, $P_{\exp }(S)$.

Knowing the maximum value of $\mathcal{K}$, we can use Eqs. (3.3) and (3.4) to express the Rabi frequency, as well as the saturation parameter, in terms of the incident photon rate $R_{\text {inc }}=P_{\text {inc }} / \hbar \omega_{0}$. As focusing from a $2 \pi$ solid angle is far more common than $4 \pi$ excitation, we consider $\mathcal{K}=2$ as the ideal case and obtain:

$$
\begin{aligned}
\Omega_{\text {ideal }} & =\sqrt{2 \Gamma_{1} R_{\text {inc }}}, \\
S_{\text {ideal }} & =\frac{4 \Gamma_{1} R_{\text {inc }}}{4 \Delta^{2}+\Gamma_{1}^{2}} .
\end{aligned}
$$

The Rabi frequency scales with the square root and the saturation parameter linearly with the incoming photon number and thus also with the incident power. To achieve a saturation parameter of $S=1$ at zero detuning, corresponding to $\Omega=\Gamma_{1} / \sqrt{2}$, we need a photon rate $R_{\text {inc }}=\Gamma_{1} / 4$, i.e., a quarter photon per lifetime. This result is valid for a coherent input state. If instead non-classical light, like photon number states or squeezed light, is used to excite the TLS, the driving strength and thus also the coupling efficiency depends furthermore on the photon statistics of the excitation field [120]. But this is beyond the scope of this work.

Using Eq. (3.7) and assuming resonant excitation, we can write:

$$
P_{\text {ideal }}(S)=S \hbar \omega_{0} \frac{\Gamma_{1}}{4} .
$$

To account for imperfect coupling, we replace the factor $\hbar \omega_{0} \Gamma_{1} / 4$ in Eq. (3.8) with $P_{S}$, which is the power necessary to drive the molecule with a saturation parameter of $S=1$ in the experiment. Hence, we obtain the following expression for $P_{\exp }(S)$ :

$$
P_{\exp }(S)=S P_{S}
$$

Now, we combine Eqs. (3.8) and (3.9) to define the coupling efficiency $\xi$ as:

$$
\xi=\frac{P_{\text {ideal }}(S)}{P_{\exp }(S)}=\frac{\hbar \omega_{0} \Gamma_{1}}{4 P_{S}} .
$$

Equation (3.10) is independent of the absolute incident power because $P_{\text {ideal }}(S)$ and $P_{\exp }(S)$ both dependent linearly on the saturation parameter. Furthermore, $\xi$ is completely defined by $P_{S}$, which is a quantity that can be easily determined experimentally with a saturation measurement [121] as discussed in the next section. 
The parameter $\xi$ characterizes how efficiently the excitation beam drives the TLS. As mentioned in the beginning of this section, only the dipole components of the incident field can drive the molecule. Hence, $\xi$ depends on the mode overlap of the focused beam and the dipolar emission of the TLS [97]. In this respect, $\xi$ is comparable to the $\beta$-factor which characterizes the coupling of an emitter to an one-dimensional waveguide structure [105, 122, 123]. While $\xi$ depends on the mode overlap of the excitation beam and the TLS emission, $\beta$ is given by the overlap of the emission and the waveguide mode. The maximum value of $\beta=1$ is achieved if all light from the emitter is collected into the waveguide. The maximum value of the coupling efficiency $\xi$ for a $2 \pi$ focusing angle, $\xi=1$, is achieved by exciting with a directional dipole wave [66]. In the uncommon situation of $4 \pi$ illumination, a value of $\xi=2$ can be realized with an electric dipole wave excitation [97].

With Eqs. (3.3), (3.4), (3.9), and (3.10) it is possible to connect scattering and coupling efficiency:

$$
\mathcal{K}=2 \frac{\xi \frac{\Gamma_{1}^{2}}{4 \Delta^{2}+\Gamma_{1}^{2}}}{\xi \frac{P_{\exp }}{\hbar \omega_{0}} \frac{4 \Gamma_{1}}{4 \Delta^{2}+\Gamma_{1}^{2}}+1} .
$$

In contrast to $\xi, \mathcal{K}$ depends not only on the mode of the incident beam but also on the detuning $\Delta$ and the absolute incoming power. In the weak excitation limit and for a resonant beam, Eq. (3.11) simplifies to $\mathcal{K}=2 \xi$.

\subsection{Measurement}

To quantify the experimental coupling efficiency we need to determine $P_{S}$. As described in subsection (2.3.2), this is done by measuring linewidth and peak fluorescence for different excitation powers (see Fig. 2.5).

Using Eq. (3.9), we can rewrite Eqs. (2.27) and (2.28) and obtain:

$$
\begin{aligned}
R_{\text {fluo }} & =R_{\max } \frac{P_{\text {inc }} / P_{S}}{1+P_{\text {inc }} / P_{S}} \\
\Gamma_{\text {FWHM }} & =2 \Gamma_{2} \sqrt{P_{\text {inc }} / P_{S}+1 .} .
\end{aligned}
$$

The saturation power $P_{S}$ is determined by fitting Eqs. (3.12) and (3.13) simultaneously to the measured values. The maximum fluorescence count rate $R_{\max }$ and the linewidth in the weak excitation limit $2 \Gamma_{2}$ are additional fitting parameters.

The incident power is measured in front of the cryostat. To obtain the excitation power at the sample, we determine the absorption by the optical 


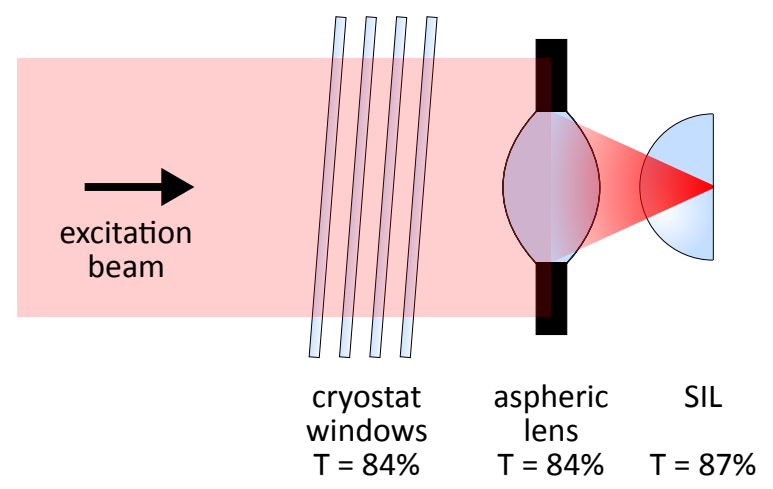

Figure 3.2: Transmission of the optical components inside the cryostat. From the initial power $61 \%$ is left, after passing through the four cryostat windows, the aspheric lens, and the solid immersion lens.
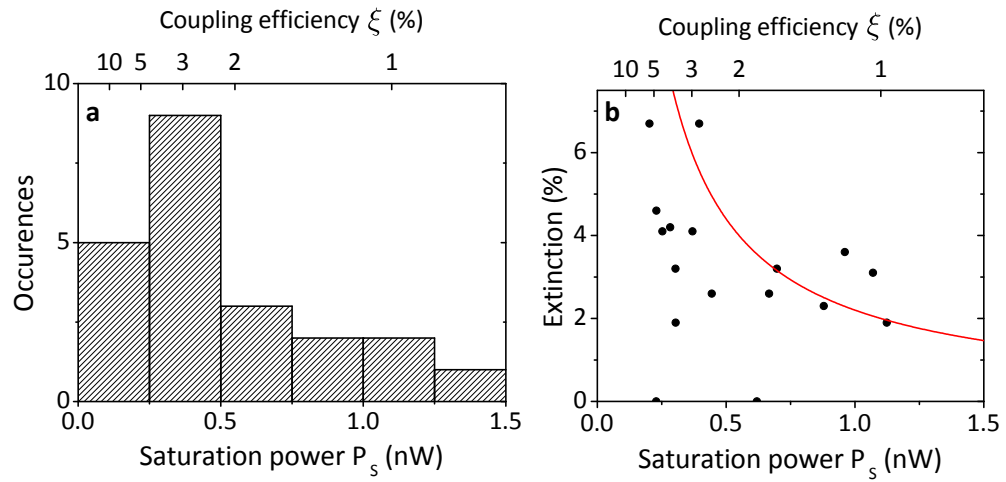

Figure 3.3: a, Distribution of saturation powers and coupling efficiencies for different DBATT molecules. b. Measured extinction values in the weak excitation limit as a function of the saturation power and coupling efficiency. The red line is a plot of Eq. (3.17)

elements inside the cryostat (see Fig. 3.2). The transmission through the SIL is calculated using the Fresnel equations for a dielectric interface. The losses at the aspheric lens are mainly caused by clipping, as the incident beam overfills the aperture of the lens. In total, $61 \%$ of the power in front of the cryostat reaches the sample.

Figure 3.3 shows a histogram of the experimentally measured values of $P_{S}$ for 23 different DBATT molecules. For each molecule focus and polarization of the incident beam are optimized. The values range from $150 \mathrm{pW}$ to $3.4 \mathrm{nW}$, with the median at $400 \mathrm{pW}$. The theoretical limit for an ideal TLS with a natural linewidth of $22 \mathrm{MHz}$, like DBATT in naphthalene, is $11 \mathrm{pW}$. Hence, our 


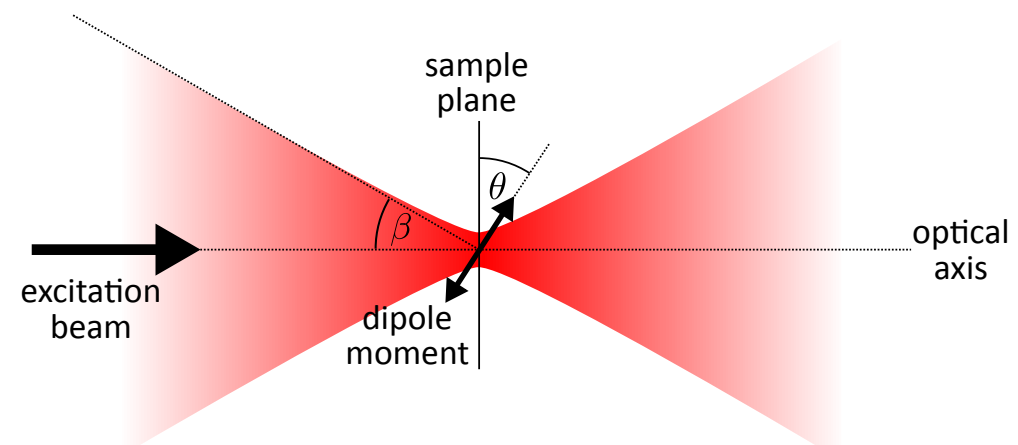

Figure 3.4: The excitation beam is focused using optics with an opening angle $\beta$ onto a single molecule. Its dipole moment is tilted out of the sample plane by an angle $\theta$.

best values correspond to a coupling efficiency of $\xi=7 \%$. However compared to an ideal TLS, the scattering cross section of the o-o ZPL is reduced by a factor of $\alpha=0.5$, due to additional decay channels of the excited state. Thus, we can optically drive with up to $14 \%$ of the maximal possible efficiency for DBATT in naphthalene.

The main factor preventing even higher coupling efficiencies is the opening angle $\beta$ of the focusing optics (see Fig. 3.4), which is $33^{\circ}$ in the experiment. Without considering the SIL, the coupling efficiency for a dipole in the sample plane $(\theta=0)$ is reduced four times compared to an opening angle of $90^{\circ}$. For a dipole moment tilted out of plane, the efficiency reduction is even higher.

Furthermore, the quality of focusing is decreased by aberrations. Ray tracing simulations of the aspheric lens and the SIL show spherical aberrations. They lead to a pronounced ring around the central maximum of the focus, as can be nicely seen from the raster scan image of a single molecule in Fig. 2.20. The intensity of the ring is up to ten times larger than the expected value for a diffraction limited Airy pattern. If the molecule is not directly on the optical axis, the SIL introduces additionally an astigmatism that further degrades the quality of the focus and thus also the coupling efficiency.

Varying out-of-plane orientation of the dipole moment can lead to a spread of saturation powers from molecule to molecule. While we account for different in-plane orientations by rotating the polarization of the incident beam in each case, the out-of-plane component cannot be optimized. Neglecting the small electric field component inside the focus which is parallel to the 
optical axis (see subsection 2.3.3), the coupling efficiency scales with the outof-plane tilt $\theta$ as $\cos ^{2}(\theta)$. Using $\theta=52^{\circ}$, as estimated in subsection 2.3.3, this factor amounts to 0.38 .

Besides the dipole orientation, also the product of Debye-Waller and Franck-Condon factor varies from molecule to molecule. We observe values between 0.3 and 0.6 , which can explain a change in saturation power by a factor of two.

It seems intuitive that the coupling efficiency $\xi$ should be connected to the extinction signal, and hence to the efficiency parameter $\eta$ which was defined in Eq. (2.42). If all incident laser light is collected in transmission, it is possible to calculate the relative size of the extinction $\operatorname{dip} V$ from the coupling efficiency [98]:

$$
V=(1-\alpha \zeta) \frac{\Gamma_{1}}{2 P_{\mathrm{inc}}} \frac{S}{S+1}=(1-\alpha \zeta) 2 \xi \frac{1}{1+P_{\mathrm{inc}} / P_{S}} .
$$

Here, $\zeta$ is the collection efficiency of the light emitted by the molecule. The requirement that all incident light should be collected in transmission imposes a lower limit on $\zeta$. For a given coupling efficiency $\xi$, there is a minimum opening angle $\beta$ of the focusing optics necessary. To collect all transmitted laser light, the detector must cover at least the same solid angle. As the molecule emits in all directions, this means that with rising $\xi$ also $\zeta$ has to increase. In particular, in the case of perfect coupling, where $\xi=\alpha=1$, the excitation light must be focused from a $2 \pi$ solid angle. Hence, the detection solid angle must also be $2 \pi$, and thus half of the light emitted by the molecule is detected, i.e., $\zeta=0.5$. If we evaluate Eq. (3.14) under these conditions and in the weak excitation limit $\left(P_{\text {inc }} \ll P_{S}\right)$, we get $V=1$ corresponding to an extinction of $100 \%$.

By comparing Eqs. (2.42) and (3.14), we can derive a relation between $\xi$ and $\eta$ :

$$
\begin{gathered}
2 \eta \frac{1}{S+1}-\eta^{2} \frac{1}{S+1}=(1-\alpha \zeta) 2 \xi \frac{1}{S+1} \\
\Rightarrow \eta=1-\sqrt{1-(1-\alpha \zeta) 2 \xi} .
\end{gathered}
$$

We discarded the non-physical solution, which would result in $\eta>1$. Equation (3.16) always has a real solution as $(1-\alpha \zeta) 2 \xi<1$. As Eq. (3.14) depends on the collection efficiency $\zeta$ of the light emitted by the molecule, the relation of $\xi$ and $\eta$ is as well a function of $\zeta$. In the special case $\zeta=\xi / 2 \alpha$, Eq. (3.16) simplifies to $\eta=\xi$.

To compare the predictions of Eq. (3.14) with our experiment, we determine the collection efficiency of molecule light in the transmission path to be $\zeta=$ 


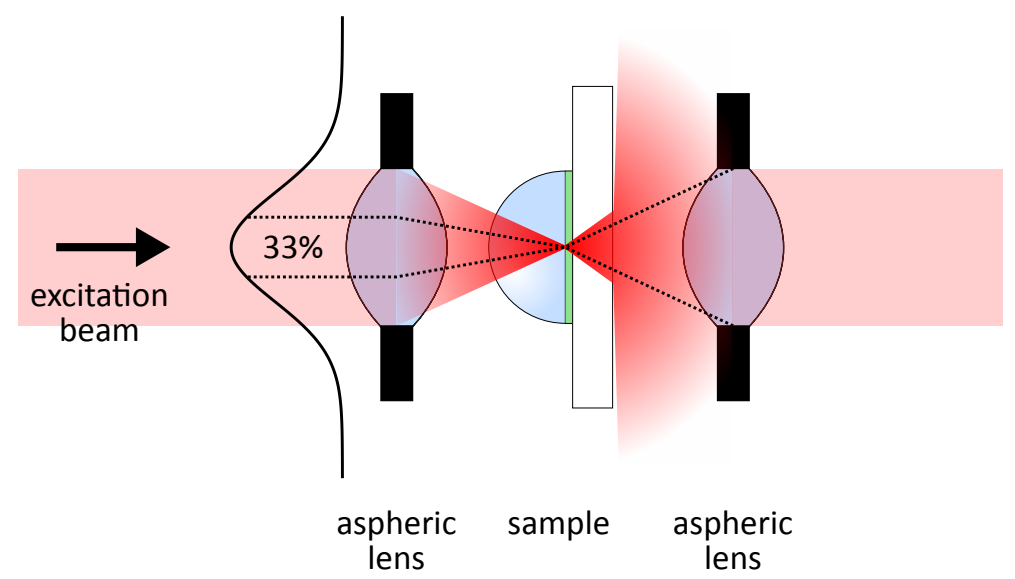

Figure 3.5: Beam path of the laser light through the optical elements inside the cryostat. The incident beam has a Gaussian intensity distribution. $90 \%$ of the beam is within the clear aperture of the first aspheric lens. Due to diffraction at the elements of the sample (SIL, naphthalene crystal, fused silica cover glass) the beam covers the full $2 \pi$ solid angle after the sample. The dashed lines indicate the critical rays that are still collected by the second asphere. $33 \%$ of the light that is within the clear aperture of the first aspheric lens is enclosed by these rays.

0.01 . We can thus approximate $(1-\alpha \zeta) \approx 1$. If we furthermore assume weak excitation, we end up with a simplified form of Eq. (3.14):

$$
V=2 \xi .
$$

Figure $3.3 \mathbf{b}$ shows measured extinction values as a function of the saturation power $P_{S}$ (bottom axis) or coupling efficiency (top axis). There is a tendency to higher extinction values as the coupling efficiency increases. However, the spread of extinction values is large and Eq. (3.17) does not fit the data very well. A possible explanation is that we do not collect all incident laser light in transmission as can be seen in Fig. 3.5. Even though the aspheric lenses used for focusing and recollimation are identical, the sample breaks the symmetry of focusing and collimation. The high refractive index of the SIL causes strong diffraction at the SIL-naphthalene interface. After the sample, the excitation beam covers the full $2 \pi$ solid angle, while the opening angle of the aspheric lens is just $33^{\circ}$. Thus, only $33 \%$ of the transmitted laser light is collected in transmission ${ }^{2}$, which violates the requirements of Eq. (3.14). A direct consequence of that is the prediction of an extinction dip

${ }^{2}$ The equality of the numerical values for the opening angle and the collection efficiency is a just a coincidence. 
larger than one, for $\xi>0.5$, according to Eq. (3.17), which is clearly not physically correct. Instead, interference of incident and scattered light occurs outside the collection angle, which leads to a difference between predicted and measured values [71].

Equation (3.14) shows that an extinction measurement can in principle be used to determine the coupling efficiency of excitation light and a single emitter. However, this is only possible if the collection efficiency of the light emitted by the molecule is known and, more importantly, all incident laser light is collected in transmission. The latter condition is not fulfilled in the experiment, and the results depicted in Fig. 3.3 b show that there is no one-to-one correspondence between the extinction signal and the coupling efficiency. Thus, we employ the saturation measurement as a more robust method to determine the light matter coupling efficiency.

\subsection{Improving the coupling efficiency}

Efficient light-matter interaction is of great importance to achieve single photon-single emitter coupling on a level sufficient for quantum information processing [26]. The comparison of our experimental results with the theoretically possible values in the previous section has shown that there is still room for improvements. Shaping of the input mode and increasing the maximum focusing angle $\beta$ are the two most obvious possibilities.

With a larger opening angle of the focus optics, the coupling efficiency could be increased up to $\xi=0.425$ ( 0.85 for a perfect dipole) if one considers a DBATT molecule with dipole parallel to the sample plane and $\beta=90^{\circ}$. If that is combined with a properly shaped input mode, the coupling efficiency reaches $\xi=0.5$ ( 1 for a perfect dipole) for an arbitrary dipole orientation [66]. State of the art commercial objectives offer opening angles of up to $72^{\circ}$. However, these microscope objectives are usually not compatible with cryogenic temperatures. Objectives specified for the use at low temperatures are commercially available with opening angles of $55^{\circ}$ (e.g., Attocube, LTAPO/VISIR/o.82). With such optics, a coupling efficiency of $\xi \approx 0.25(0.5$ for a perfect dipole) would be feasible [66].

These calculations assume a dipole in vacuum. In the experiment, however, the molecule is located close to an interface between two dielectric media, which greatly influences the emission pattern as depicted in Fig. 3.6. We extend the calculation of coupling efficiencies to this more general case by using the argumentation of Ref. [97]: Only the part of the incoming wave 

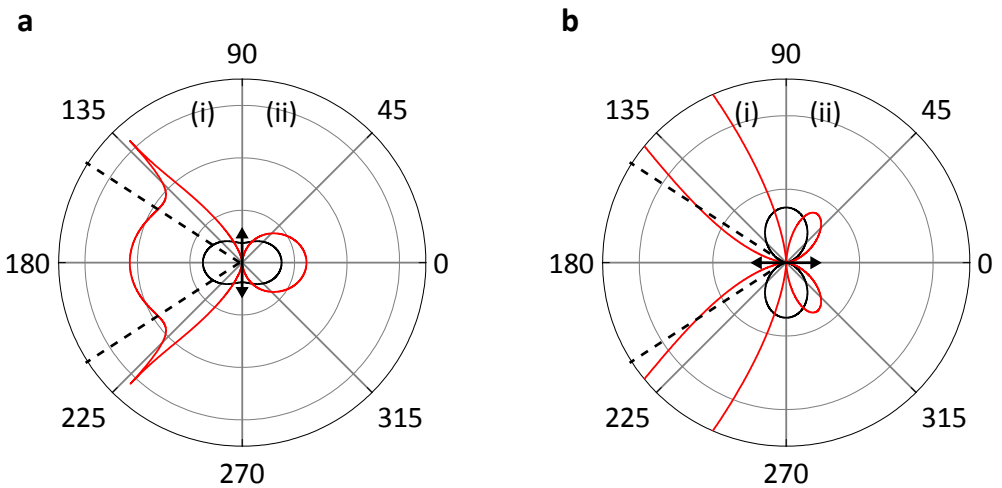

Figure 3.6: Dipole emission pattern in vacuum (black line) and at a dielectric interface (red line). The half-spaces with different media are indicated by (i) and (ii). In case of the black lines (i) and (ii) are vacuum, while for the red lines (i) is cubic zirconia and (ii) is naphthalene. In the latter case, the dipole is inside the naphthalene, $50 \mathrm{~nm}$ away from the interface. The dashed lines mark the collection angle covered by the aspheric lens. In a, the dipole moment is parallel to the interface, while it is perpendicular in $\mathbf{b}$. The displayed patterns are integrated over a $2 \pi$ rotation around the $0^{\circ}$ axis. In $\mathbf{b}$, the red case is truncated.

overlapping with field emitted by the dipole can excite the system. This overlap is, of course, optimized if the incoming wave is equal to the emitted wave but with reversed propagation direction. If the incoming field is truncated to a limited solid angle, the optimized overlap is given by the fraction of light emitted into this solid angle. Hence, there is a direct correspondence between collection and excitation efficiency $\xi$. As we used the maximum possible excitation for light incident from a $2 \pi$ solid angle for the definition of $\xi$ in Eq. (3.10), $\xi$ is equal to twice the collection efficiency. The maximum value was so far $\xi=1$, as at most $50 \%$ of the emitted light can be collected from a dipole in vacuum within a $2 \pi$ solid angle. At a dielectric interface, however, it is possible to collect more than half of the emitted light. In particular, it is attainable with a properly designed multilayer structure, to direct more than $99 \%$ of the emission into a solid angle, which can be covered by commercial high-NA microscope objectives [124, 125]. This would lead almost to the perfect coupling efficiency of $\xi=2$ for a properly shaped incident beam and a perfect emitter.

Returning to Fig. 3.6, we can see how the dielectric interface affects the coupling efficiency. The dipole emits predominantly into the medium of lager refractive index [126] $]^{3}$. The amount of light collected by the aspheric lens

3 The formulas presented in the appendix of Ref. [126] for the calculation of the dipole emission patterns are not fully correct. Using the principles described in that paper and the 
is reduced from $11 \%$ to $4 \%$, for a dipole parallel to the interface, and from $2 \%$ to $0.3 \%$, for a perpendicular dipole moment if the molecule is embedded in naphthalene instead of vacuum. Following the correspondence principle described in the previous paragraph, the maximum possible $\xi$ is thus reduced from 0.22 to 0.08 and from 0.04 to 0.006 for the respective dipole orientations. By interfacing naphthalene to a material with larger refractive index, the dipole emission can be directed more towards the collection angle, as shown by the red lines in Fig. 3.6 for cubic zirconia. In that case, the coupling efficiency can reach 0.42 and 0.15 , for parallel and perpendicular dipoles, respectively.

The coupling efficiency can be increased even more if instead of cubic zirconia another material with higher refractive index is employed. In the following subsection, we show first experimental data, where a gallium phosphide SIL with a refractive index of 3.33 [128] is used to increase the fraction of collected photons. In the second subsection, we demonstrate how a spherical micro-mirror can increase the light-matter coupling efficiency.

\subsubsection{Gallium phosphide SIL}

Gallium phosphide ( $\mathrm{GaP}$ ) is an indirect band-gap semiconductor, which is transparent in the visible for light with a wavelength longer than $554 \mathrm{~nm}$. Its high refractive index, $n=3.33$ at $619 \mathrm{~nm}$, makes it a highly attractive SIL material [128].

Figure 3.7 shows how the dipole emission pattern is changed from cubic zirconia (black) to $\mathrm{GaP}$ (red). For either dipole orientation, the emission maximum occurs at smaller angles, as the critical angle for total internal reflection at the SIL-naphthalene interface, is reduced from $47^{\circ}$ to $28^{\circ}$. Parts $\mathbf{c}$ and $\mathbf{d}$ show that this greatly improves the collection efficiency as $28^{\circ}$, in contrast to $47^{\circ}$, lies within the solid angle covered by the aspheric lens. Depending on the distance between molecule and interface, and the dipole orientation, the collection efficiency can reach almost $45 \%$ for a parallel dipole $50 \mathrm{~nm}$ from the interface, and even $65 \%$ for a dipole perpendicular to the interface at a distance of $120 \mathrm{~nm}$.

The interface also influences the decay rate of the molecule (dashed curves in $\mathbf{c}$ and d), which can be enhanced more than five times for dipoles very close to the interface. However, this additional emission occurs predominantly in directions larger than the critical angle, which are not covered by the aspheric

expressions for the fields at dielectric interfaces (see, e.g., Ref. [127]), the correct formulas can be calculated. 
a

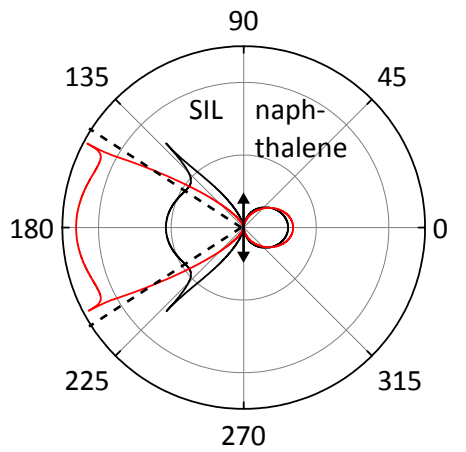

b

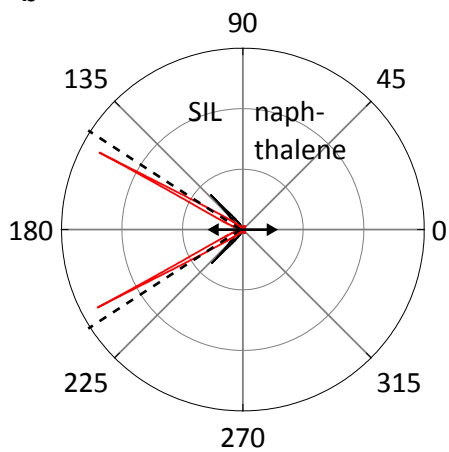

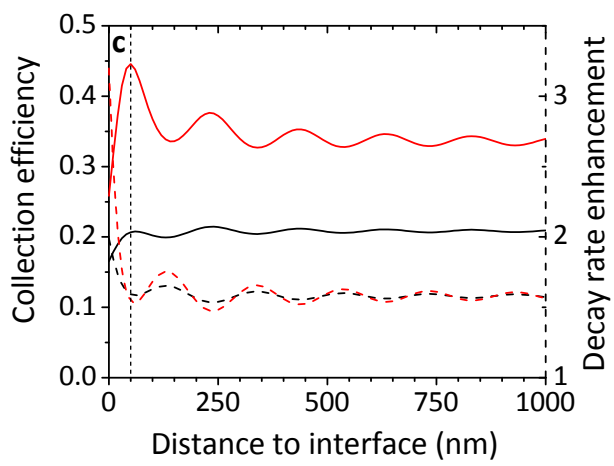

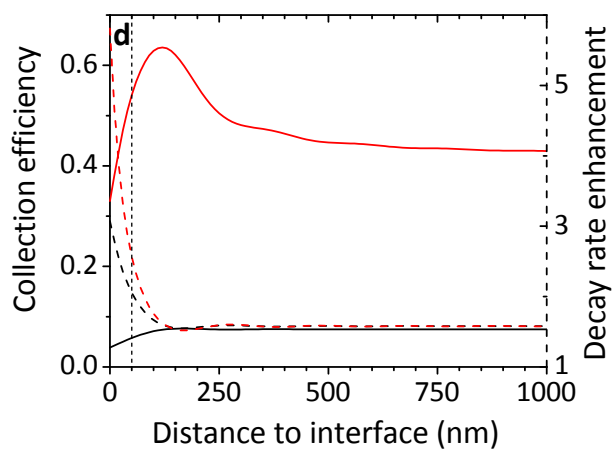

Figure 3.7: Comparison of cubic zirconia (black lines) and GaP SIL (red lines). a and b, Emission pattern of a dipole $50 \mathrm{~nm}$ away from the interface. The dipole orientation is indicated by the arrow and the dashed lines show the collection angle of the aspheric lens. c, The solid lines show the collection efficiency of the light emitted by a dipole parallel to the interface as a function of the dipole-interface distance. The dashed lines, which are projected on the righthand axis, display the enhancement of the decay rate, normalized to the vacuum value. The vertical dashed line, denotes the $50 \mathrm{~nm}$ distance used for calculating the emission patterns in $\mathbf{a}$ and $\mathbf{b}$. $\mathbf{d}$, same as $\mathbf{c}$, but for a perpendicular dipole.

lens, leading to a reduction of the collection and thus also the coupling efficiency.

$\mathrm{GaP}$ is a rather soft material and produces toxic gas during polishing [96]. Thus, it is very challenging to manufacture GaP optics and our SILs (A.W.I.) are consequently not of very high quality. In particular, their surface is very rough. Atomic force microscopy of the flat side and interferometric measurements of the curved side revealed irregularities of several $100 \mathrm{~nm}$. Besides the negative impact on focusing and imaging quality, this prevents usage of thin channels, as it is impossible to get the SIL into optical contact with the cover glass. Additional polishing does not yield any improvements, as even the finest polishing emulsion causes deep scratches inside the soft GaP. 

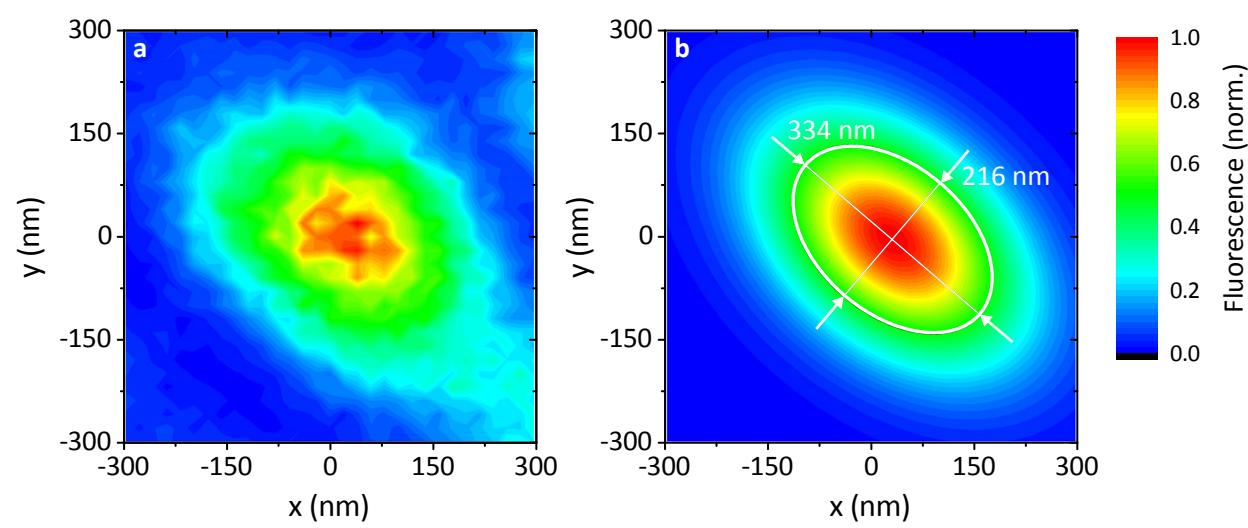

Figure 3.8: Laser raster scan image of the fluorescence signal of a single molecule using a GaP SIL. a shows the data, while $\mathbf{b}$ is a two dimensional Gaussian fit to $\mathbf{a}$. The ellipse labels the half maximum position. The FWHM along short and long axis is 216 and $334 \mathrm{~nm}$, respectively.

Despite the low quality of the GaP SILs, we prepared a sample to investigate the collection efficiency. We placed a small flake of DBATT in naphthalene between a cover glass and a GaP SIL, and pressed the SIL against the cover glass with a thin aluminum band. Subsequent melting and re-solidifying of the naphthalene lead to a crystal of undefined thickness, covering the whole field of view. Optical alignment of the GaP SIL is more difficult than of the cubic zirconia SIL for two reasons: Firstly, the curved side of the GaP SIL is anti-reflection coated. Hence, we could not use the curved side reflection to center the SIL on the optical axis, as described in subsection 2.2.2. Instead, we optimized the lateral position of the SIL by monitoring the shape of the laser focus. Secondly, GaP is more sensitive to misalignment due to its larger refractive index.

Figure 3.8 displays a laser scan image of a single molecule through a $\mathrm{GaP}$ SIL. The focal spot is clearly elliptic, which is probably caused by a small lateral deviation from the optical axis introducing an astigmatism. While the FWHM of the large axis is comparable to the values achieved with a cubic zirconia SIL, the short axis has a FWHM of only $216 \mathrm{~nm}$ - very close to the theoretical limit of $196 \mathrm{~nm}$. This demonstrates that the focal spot could be decreased by about one third with a GaP SIL, and how critical proper alignment and working close to the optical axis is. With a GaP SIL, the total internal reflection angle is within the aperture covered by the aspheric lens. Hence, we realize the maximum possible NA inside naphthalene of 1.58. Using a different SIL material with an even higher refractive index, or a lens with 

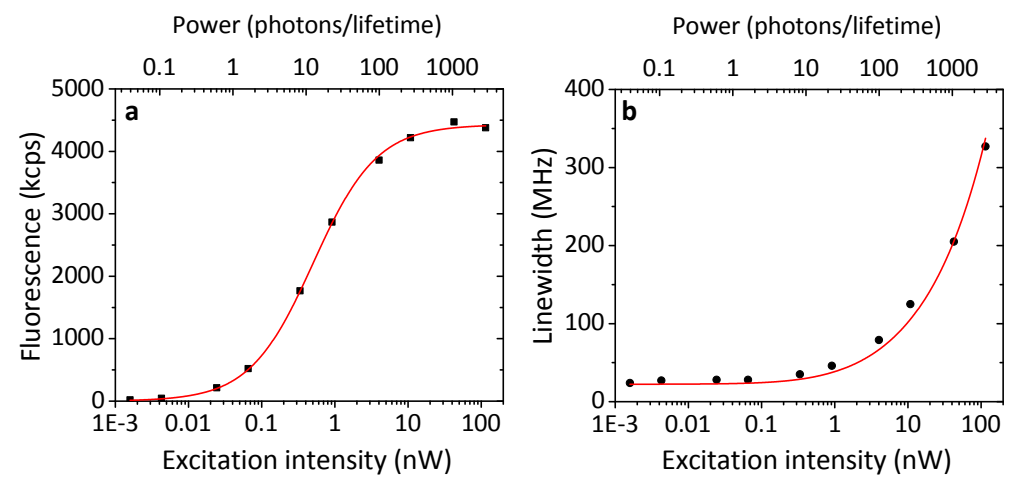

Figure 3.9: Saturation measurement of a single DBATT molecule, using a GaP instead of a cubic zirconia SIL. a, Peak fluorescence count rate in kilo counts per second (kcps). b, Transition linewidth for different excitation power. The solid red line is a simultaneous fit to the data of $\mathbf{a}$ and $\mathbf{b}$. The excitation power is corrected for the transmission of the elements inside the cryostat and thus corresponds to the excitation power at the sample. A background linear in the incident power, is removed from the count rate to account for off-resonant excitation of other molecules. The fit yields a saturation power of $507 \mathrm{pW}$ or 11.4 photons per lifetime.

larger NA would thus not decrease the size of the focal spot any further, but could still improve coupling and collection efficiency.

We performed a saturation measurement (see Fig. 3.9) to deduce coupling and collection efficiencies. As mentioned earlier, the curved side of the GaP SIL is anti-reflection coated, thus we assume that the transmission of this interface is $100 \%$. But at the GaP-naphthalene interface, we lose around $13 \%$ due to reflection ${ }^{4}$. Together with the transmission losses at the cryostat windows and the aspheric lens (compare Fig. 3.2) we end up with a correction factor for the incident power of 0.61 . The fit yields a coupling efficiency of roughly $2 \%$, which is within the range of efficiencies measured with cubic zirconia SILs. The maximum count rate, is 4.4 Mcps and thus about three times larger than the rates we typically measure with cubic zirconia SILs. This increase can be well explained by the improvement in collection efficiency, which ranges between a factor of 1.6 and 5.7 depending on the dipole orientation. The fluorescence rate was corrected for the nonlinearity of the SPCM at high count rates, assuming a dead-time of $45 \mathrm{~ns}$ [71]. Furthermore, we removed a background linear in the incident power caused by the off-resonant excitation of other molecules.

\footnotetext{
${ }^{4}$ As the sample is thick, we assume that there is negligible excitation of the molecule via evanescent fields.
} 
Experimental determination of the collection efficiency requires a careful characterization of the optical losses on the way from the sample to the detector, which amount to $44 \%$. The detection efficiency of the SPCM module is $65 \%$ according to the data sheet. As the Franck-Condon factor was not measured for this molecule, we assume $\alpha_{\mathrm{FC}}=0.6$ corresponding to smallest values we measured for DBATT in naphthalene, to establish a lower bound for the collection efficiency. The maximum possible count rate of red-shifted photons is thus:

$$
R_{\text {red }}=\underbrace{0.5 \cdot 2 \pi \cdot 22.4 \mathrm{MHz}}_{\text {Maximum emission rate }} \cdot \underbrace{(1-0.6)}_{\text {fraction of red-shifted photons }} \cdot 0.56 \cdot 0.65=10.2 \mathrm{MHz} .
$$

Comparison of $R_{\text {red }}$ with the measured fluorescence rate, yields a collection efficiency of $43 \%$. As the linewidth in the weak excitation limit is not broadened compared to the value measured in bulk naphthalene and the sample is thick, we conjecture that the molecule is several $100 \mathrm{~nm}$ away from the interface. At that distance, such a high collection efficiency can be only explained with a perpendicular dipole orientation (see Fig. 3.7 d). This orientation could also explain the weak coupling of the incident field to the molecule: The emission pattern of a perpendicular dipole has two sharp peaks (see Fig. 3.7 b) and hence the overlap with Gaussian input beam is very small.

These measurements show that a GaP SIL has the potential to significantly increase the light-matter coupling efficiency. But to make full use of the benefits, higher quality SILs are required, such that samples of well-defined thickness can be made to exploit the collection efficiency peak between 50 and $120 \mathrm{~nm}$. Furthermore, proper shaping of the incident field, e.g., with a spatial light modulator, is necessary, to match the dipole emission pattern. If these conditions are fulfilled, coupling efficiencies exceeding $\xi=0.6$ (assuming $\alpha \approx 0.5$ ) should become possible.

\subsubsection{Spherical mirror}

Even with a GaP SIL, a significant fraction of the light is emitted into the host crystal and thus not collected. To remedy that problem, we fabricated a sample with spherical micro-mirrors. If a molecule is in the center of such a mirror, the emitted light that would be lost without the mirror is reflected into the excitation direction and can be collected. At the same time, the excitation beam is reflected by the mirror and refocused onto the molecule. Hence, we expect a significant improvement of the coupling efficiency.

Figure 3.10 a shows a schematic of the fabrication process. First, a polystyrene film is prepared on a teflon substrate. Subsequently, silica 
a

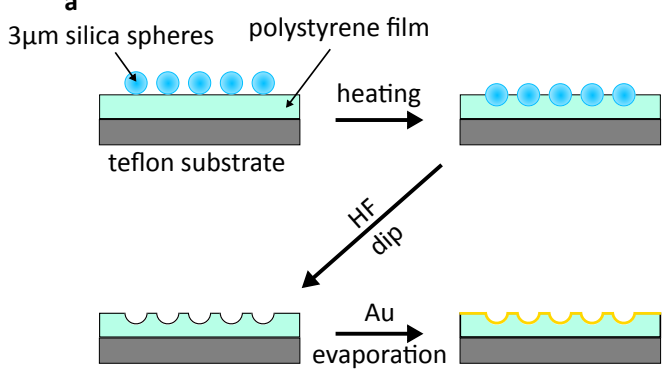

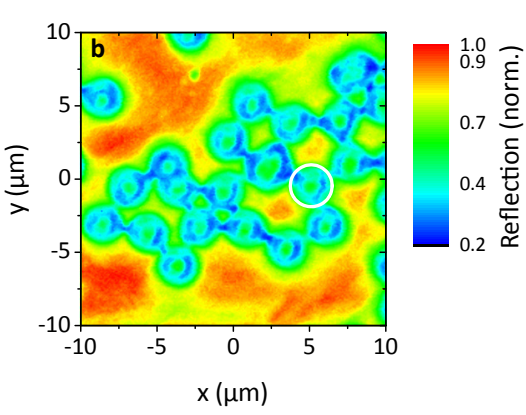

Figure 3.10: a, Fabrication of the micro-mirrors. b, Laser raster scan image of several mirrors on the sample. The encircled mirror is shown again in Fig. 3.11

spheres with a $3 \mu \mathrm{m}$ diameter are dispersed on the polystyrene by dropcasting an aqueous solution. The concentration is adjusted such that no multi-layers of silica spheres form after evaporation of the water. In the next step, the substrate is heated to $200{ }^{\circ} \mathrm{C}$, which softens the polystyrene, and the silica beads start sinking into the film. Every minute we measure how far the spheres have migrated into the polystyrene with an atomic force microscope. The heating is stopped when the spheres are half embedded. Afterwards, the silica beads are removed by dipping the sample into hydrofluoric acid for one minute. In the last step, a $100 \mathrm{~nm}$ thick gold layer is evaporated on top of the sample. The result are many sparsely scattered micro-mirrors, which have their center approximately in the crystal plane (see Fig. 3.10 b).

A small droplet of a DBATT in n-tetradecane solution ${ }^{5}$ was cast on the mirrors, and the cubic zirconia SIL placed on top and fixed mechanically with a thin plastic band.

Inside the cryostat, we searched for molecules close to the center of a micro-mirror. Figure 3.11 a shows a laser scan image of a DBATT molecule $230 \mathrm{~nm}$ away from the mirror center (indicated by the black circle). The position of the latter can be nicely determined by measuring the laser power reflected of the sample. As the laser is incident on the center of the mirror, the reflected laser light is refocused and collimated by the aspheric lens. Thus, the center appears as region of increased reflectivity (see Fig. 3.11 b).

To investigate the impact of the micro-mirror on the coupling efficiency, we determined the saturation power of the molecule as shown in Fig. 3.11. Fitting the theoretical model to the data displayed in Fig. 3.12 yields a saturation

5 The properties of DBATT in n-tetradecane and DBATT in naphthalene are very similar. See, e.g., Ref. [96] for details. 

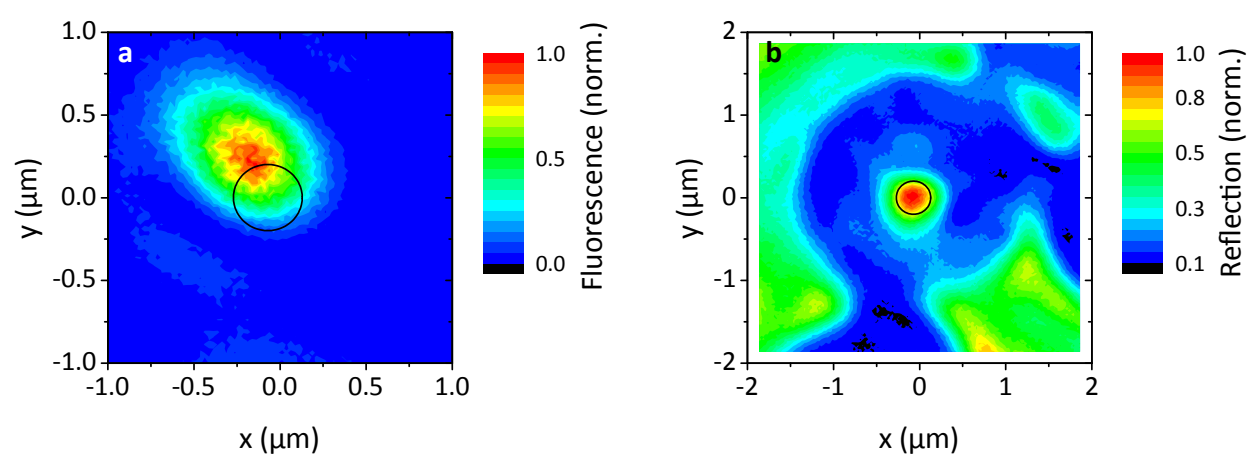

Figure 3.11: Laser raster scan images of the fluorescence of single molecule (a) and the reflection of a spherical micro-mirror (b). The black circle shows the position of the mirror focus in both images. The molecule is $230 \mathrm{~nm}$ away from the focus center. Please note that the lateral dimensions differ for $\mathbf{a}$ and $\mathbf{b}$.

power of just $81 \mathrm{pW}$, corresponding to only 1.9 photons per lifetime. Hence, the coupling efficiency is $13 \%$ - an improvement of almost a factor of two compared to the values we achieve without mirror (see Fig. 3.3).

Owing to the large coupling efficiency, we can also nicely observe the coherent interaction of incident light and molecule. As the curved mirror obstructs the transmission path, we cannot perform a usual extinction measurement but instead monitor the reflected laser power, when the excitation beam is focused at the molecule [96]. Hereby, an iris is used in the detection path to suppress light that is not scattered directly from mirror or molecule. The origin of the signal shown in Fig. 3.13 is similar to the mechanism which causes extinction: Laser light that is reflected off the sample interferes destructively with light coherently scattered by the molecule [96]. The result is a drop in reflected power of almost $40 \%$ induced by a single molecule.

A natural extension of this work would be the combination of mirror and GaP SIL. In principle, this should enable excitation from almost the full $4 \pi$ solid angle - similar to the experiment envisioned in Ref. [129].

Another promising approach is the implementation of the dielectric antenna structure presented in Ref. [125]. Using standard optical lithography, it should be possible to fabricate such a structure inside a nano-channel. When using a GaP SIL as topmost layer, the collection efficiency should be close to unity, even with the limited NA of our aspheric lens. Consequently, we could achieve, with a correctly shaped incident beam, coupling efficiencies around $\xi=1$, limited only by the branching ratio of the o-o ZPL. 

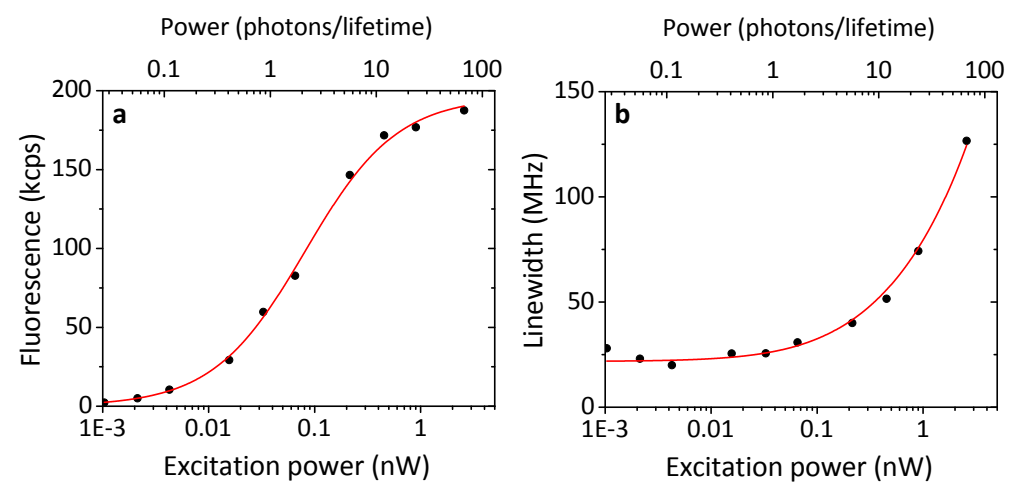

Figure 3.12: Saturation measurement of a single DBATT molecule in a n-tetradecane matrix in front of a spherical micro-mirror. a, Peak fluorescence count rate in kilo counts per second (kcps). b, Transition linewidth for different excitation powers. The solid red line is a simultaneous fit to the data of $\mathbf{a}$ and $\mathbf{b}$. The excitation power is corrected for the transmission of the elements inside the cryostat and thus corresponds to the excitation power at the sample. The fit yields a saturation power of $81 \mathrm{pW}$.

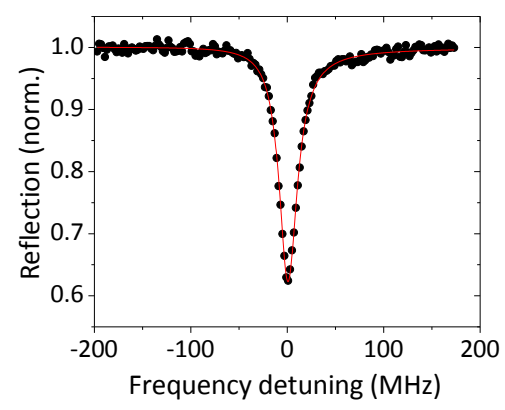

Figure 3.13: Reflected laser power with the incident beam focus placed on a single molecule. 



\section{Nonlinear optics with a single molecule}

A single molecule responds intrinsically in a nonlinear fashion to the intensity of an incident laser beam. As described in chapter 2, the population in the excited state and thus the rate of emitted photons approaches a maximum value that cannot be overcome by increasing the pump power further. This phenomenon, known as saturation, has its origin in the properties of a TLS: once the molecule is in the excited state it cannot be excited any further. Only after relaxation to the ground state, via stimulated or spontaneous emission, an incident photon can again transfer the molecule into the excited state. The measurements presented in chapter 3 demonstrate that we can enter this nonlinear regime already at very weak excitation powers of less than one photon per excited state lifetime owing to the efficient coupling of light and molecule.

This nonlinear response can be exploited to mediate an interaction of two light fields. The transmission signal of a weak beam shows extinction when its frequency coincides with the resonance of the molecule. If a second intense light field saturates the molecule, this extinction signal will be reduced or even disappears completely. In that way, the strong beam can be used to control the transmission of another light field. If furthermore, both beams have different frequencies, we observe nontrivial nonlinear effects beyond saturation: an AC-Stark shift of the molecular resonance frequency and energy transfer from one beam to the other via stimulated Rayleigh scattering or three-photon amplification.

While the nonlinear interaction of two near resonant beams with a TLS was already studied in the 1970s, it required large ensembles of atoms in the gas phase to yield a measurable signal [130-132]. Later on, experiments were performed on a single molecule [24, 25, 84], where the nonlinear interaction was demonstrated by analyzing changes in the fluorescence signal caused by the presence of the pump beam. A coherent observation of the transmitted probe beam intensity was made with a single quantum dot [133, 134], albeit the efficiency was very weak such that lock-in techniques were required to extract the signal from the noise floor.

In this chapter, we demonstrate optical control of the probe transmission spectrum by using a pump beam with a power of only a few tens of nano-watt. 
Notably, the pump can switch the transmission of the probe beam on or off, and the signal variation is large enough that it can be measured without the need of noise suppressing techniques. Such nonlinear interaction of weak fields, which would ideally contain only single photons, is of particular interest to the field of quantum information processing [26]. Furthermore, we report the observation of the time resolved fluorescence signal, illustrating the coherent driving of the excited state population.

In the first section of this chapter, we employ the dressed atom picture to provide a qualitative understanding of the physical effects occurring, when two beams interact with a TLS. In the next section, we extend the optical Bloch equations from subsection 2.1.4 to the situation of two excitation beams. We use the resulting equations to derive the steady-state transmission and fluorescence signals as well as the time-dependent excited state population. In the third and forth sections, we present the experimental results on the pump beam controlled transmission spectra and discuss a potential application of a single molecule as a few-photon switch, respectively. In the last section we show time-dependent measurements of the transmission and fluorescence signals.

\subsection{Probing a dressed two-level system}

Investigating the nonlinear interaction of a strong pump and a weak probe beam with a single molecule requires efficient coupling of both beams to the emitter. To that end, they should have frequencies close to the resonance of the molecule. While the pump beam with frequency $\omega_{\text {pmp }}$ is kept at a fixed detuning $\Delta=\omega_{\mathrm{pmp}}-\omega_{0}$, the frequency of the probe beam $\omega_{\mathrm{prb}}$ is scanned across the resonance to interrogate the properties of the combined pump beam-molecule system.

A convenient way to visualize the impact of a strong pump beam on the response of a TLS is the dressed state picture [9o]. In this approach, we assume that the TLS is coupled to a single laser mode, populated with a number of pump photons $N$, which is distributed around an average value $\langle N\rangle$, with a width of $\Delta N$. The states $|g\rangle$ and $|e\rangle$ (see Fig. 4.1 a) of the TLS and the number of photons in the laser mode are combined to form an infinite ladder of energy states. If $\omega_{\text {pmp }}$ is close to the resonance frequency $\omega_{0}$, the state $|g, N+1\rangle$, where the TLS is in the ground state and $N+1$ photons are in the laser mode, and state $|e, N\rangle$, where the TLS is in the excited state and $N$ photons populate the laser mode, are nearly degenerate (see Fig. 4.1 b). Via absorption and stimulated emission of pump laser photons, the system 
a

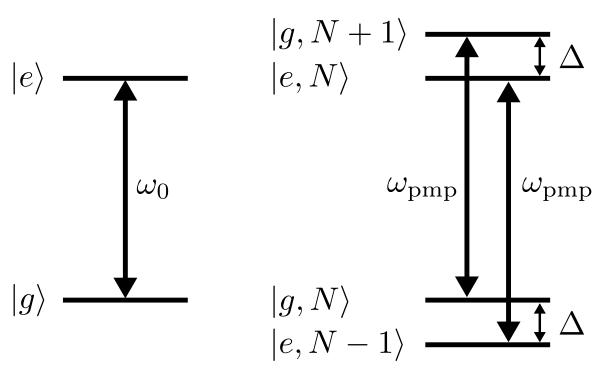

C

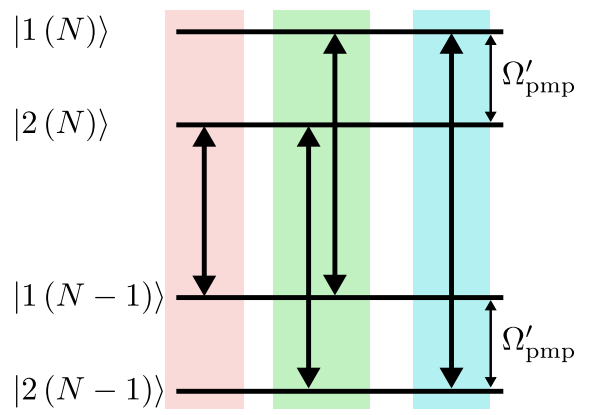

Figure 4.1: a, Level scheme of the TLS. b, Four energy levels out of the infinite ladder of states of TLS and pump photons in the uncoupled basis. c, The same four levels in the dressed state picture. The arrows indicate the possible dipole transitions. The colors label transitions at $\omega_{\mathrm{pmp}}-\Omega_{\mathrm{pmp}}^{\prime}$ (red), $\omega_{\mathrm{pmp}}$ (green) and $\omega_{\mathrm{pmp}}+\Omega_{\mathrm{pmp}}^{\prime}$ (blue).

can be transferred between these two states, which are thus no eigenstates of the Hamiltonian describing the system. The coupling strength of the two states is proportional to $\sqrt{N+1}$. In the limit of high laser mode occupation, $\langle N\rangle \gg \Delta N$ and thus the coupling strength is almost constant over a range of $\Delta N$ around the average photon number. The eigenstates of the combined pump beam and TLS system are [90]:

$$
\begin{aligned}
& |1(N)\rangle=\sin \theta|g, N+1\rangle+\cos \theta|e, N\rangle, \text { and } \\
& |2(N)\rangle=\cos \theta|g, N+1\rangle-\sin \theta|e, N\rangle, \\
& \text { with } \tan 2 \theta=-\frac{\Omega_{\mathrm{pmp}}}{\Delta} ; \quad 0 \leq 2 \theta<\pi,
\end{aligned}
$$

and $\Omega_{\mathrm{pmp}}$ is the pump field Rabi frequency. The coupled states are coherent superpositions of the TLS ground and excited states. Depending on $\Omega_{\mathrm{pmp}} / \Delta$, the state $|1(N)\rangle$ has either more characteristics of the ground or of the excited state, while the opposite is true for $|2(N)\rangle$. The two states $|1(N)\rangle$ and $|2(N)\rangle$ have an energy splitting given by the generalized pump Rabi frequency:

$$
\Omega_{\mathrm{pmp}}^{\prime}=\sqrt{\Delta^{2}+\Omega_{\mathrm{pmp}}^{2}}
$$

Figure 4.1 c depicts the energy levels of the dressed states and the allowed dipole transitions between the respective levels.

The dressed TLS can be investigated by measuring the transmission spectrum of a weak probe beam. To gain a qualitative understanding of this signal, 


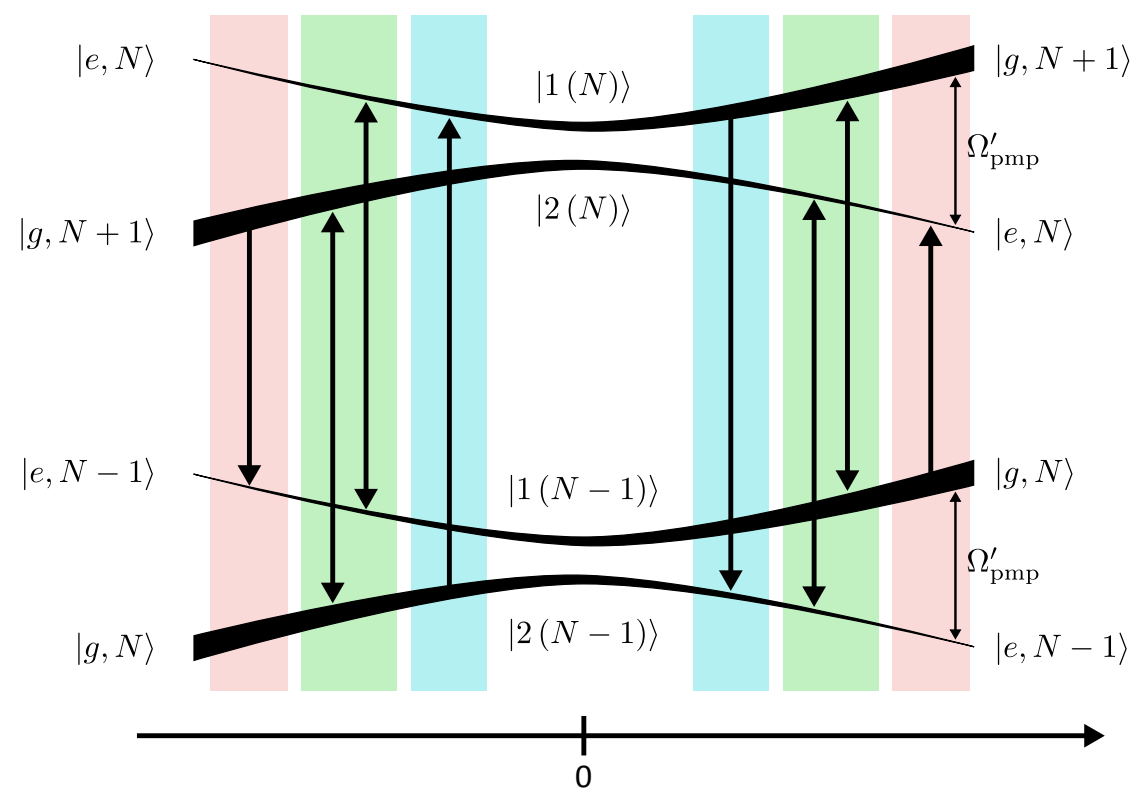

Pump frequency detuning $\Delta$

Figure 4.2: Position of the dressed state energy levels and their steady state population for varying pump detuning $\Delta$. The line thickness indicates the steady state population of the various levels. The states to the left and to the right indicate the asymptotic behavior of the dressed states for large positive and negative detunings, respectively. The colors indicate the transitions at $\omega_{\mathrm{pmp}}-\Omega_{\mathrm{pmp}}^{\prime}($ red $), \omega_{\mathrm{pmp}}$ (green) and $\omega_{\mathrm{pmp}}+\Omega_{\mathrm{pmp}}^{\prime}$ (blue). Figure adapted from [135].

it is instructive to look at the steady-state population $\pi$ of the dressed states given by [9o]:

$$
\begin{aligned}
& \pi_{1}^{\text {st }}=\frac{\sin ^{4} \theta}{\cos ^{4} \theta+\sin ^{4} \theta} \text { and } \\
& \pi_{2}^{\text {st }}=\frac{\cos ^{4} \theta}{\cos ^{4} \theta+\sin ^{4} \theta} .
\end{aligned}
$$

Figure 4.2 shows the dressed state population as a function of the pump detuning $\Delta$. The four levels are connected via three different transition frequencies, $\omega_{\text {pmp }} \pm \Omega^{\prime}$ and $\omega_{\text {pmp }}$ that can be studied by tuning the probe beam to the respective frequency.

The transition at $\omega_{\text {pmp }}-\Omega^{\prime}$ (red) connects the levels $|1(N-1)\rangle$ and $|2(N)\rangle$. If the pump detuning $\Delta>0$ (right hand side of Fig. 4.2), the population in the lower state is larger than the population in the upper state. The weak 
probe beam excites the system and the transmitted signal shows extinction. But the maximum attenuation, which occurs at $\omega_{0}$ without the pump beam, appears now at a frequency of $\omega_{\mathrm{pmp}}-\Omega^{\prime}$, which is known as the AC-Stark effect or light shift.

If $\Delta<0$ (left hand side of Fig. 4.2), however, the population in the upper state is larger than the population in the lower state. The weak probe beam will thus experience amplification via stimulated emission from state $|2(N)\rangle$ to $|1(N-1)\rangle$. In the asymptotic limit of large pump detuning, this corresponds to the transition from $|g, N+1\rangle$ to $|e, N-1\rangle$, i.e., the number of pump photons is reduced by two, while the TLS is transfered from the ground into the excited state. Hence, there are in total three photons involved in this process: the absorption of two pump photons and the stimulated emission of one probe photon. This process is known as "three-photon amplification" [136] or "hyper-Raman transition" [135, 137].

If we look at the $\omega_{\text {pmp }}+\Omega^{\prime}$ transition (blue), the opposite is true: the probe beam experiences attenuation due to the AC-Stark shift of the resonance for $\Delta<0$ and three-photon amplification for $\Delta>0$.

The interpretation of the transition at $\omega_{\text {pmp }}$ (green) is more involved. It couples the state $|2(N-1)\rangle$ with $|2(N)\rangle$ and $|1(N-1)\rangle$ with $|1(N)\rangle$, respectively. Independently of the detuning $\Delta$, the populations in the lower and upper state are equal. From the simple population difference argument used before, one would thus expect that the probe beam is not affected at all at this frequency. If we consider the asymptotic limit, the two transitions at $\omega_{\text {pmp }}$ connect the states $|g, N\rangle$ with $|g, N+1\rangle$ and $|e, N-1\rangle$ with $|e, N\rangle$, respectively. The change in the number of pump photons suggests that, as in the three-photon amplification case, a combination of pump and probe photons is involved. Indeed, during this "stimulated Rayleigh scattering" process, energy is transfered from pump to probe beam and vice versa [131]. The effect has its origin in the interference of different two and four photon transitions and is discussed in detail in Ref. [138]. It results in a dispersive feature centered around the pump beam frequency $\omega_{\text {pmp }}$ that can either lead to amplification or attenuation of the probe beam.

Figure 4.3 a shows the probe beam transmission spectrum as a function of the pump-probe detuning $\delta=\omega_{\text {prb }}-\omega_{\text {pmp }}$, in the case of $\Delta>0$. The three processes mentioned in the previous paragraphs can be nicely seen: an absorptive feature at $\delta=-\Omega_{\mathrm{pmp}}^{\prime}$ (red area), a dispersive region around $\delta=0$ (green area) and amplification for $\delta=\Omega_{\mathrm{pmp}}^{\prime}$.

If $\Delta \approx 0$, the populations of all dressed states are equal (see central region of Fig. 4.2). Hence, it is no longer clear whether the transitions at 

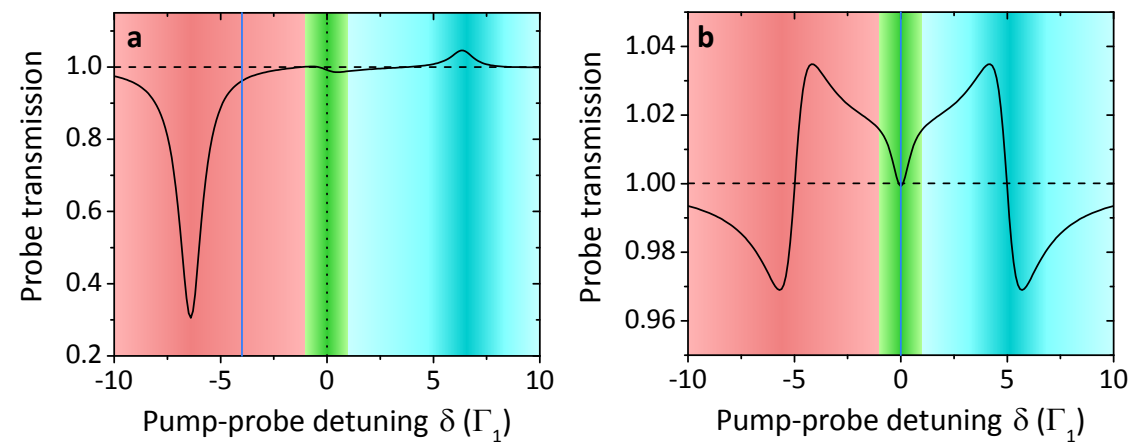

Figure 4.3: Theory curve of the probe beam transmission spectra. Parameters are $\Omega_{\mathrm{pmp}}=5 \Gamma_{1}$, $\Omega_{\mathrm{prb}}=0.1 \Gamma_{1}$ and $\Delta=4 \Gamma_{1}(\mathbf{a})$ or $\Delta=0(\mathbf{b})$. The vertical solid blue and dotted black lines indicate the transition frequency of the unperturbed molecule and the pump beam frequency, respectively.
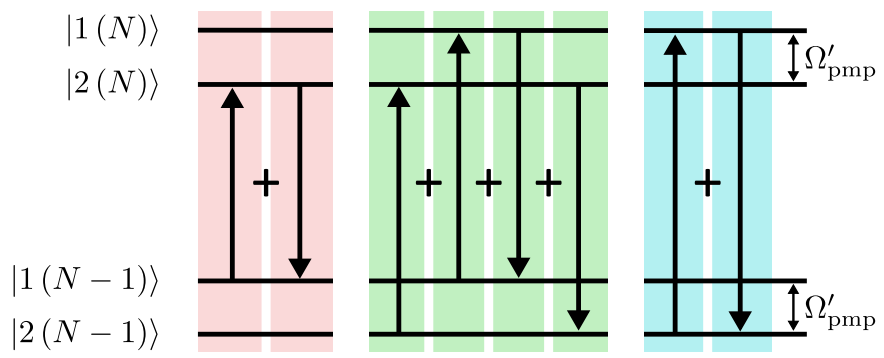

Figure 4.4: Dressed state picture in the case of $\Delta=0$. The population of all states is equal. The color coding indicates the frequency of the probe beam: $\omega_{\mathrm{pmp}}-\Omega_{\mathrm{pmp}}^{\prime}$ (red), $\omega_{\mathrm{pmp}}$ (green) and $\omega_{\mathrm{pmp}}+\Omega_{\mathrm{pmp}}^{\prime}$ (blue).

$\omega_{\text {pmp }} \pm \Omega_{\text {pmp }}^{\prime}$ show absorption or amplification. As visualized in Fig. 4.4, both processes occur at the same time and add up coherently, resulting in a complex probe beam transmission line shape as displayed in Fig. 4.3 b. There are two dispersive features (red and blue) which reach a transmission equal to 1 at $\delta= \pm \Omega_{\mathrm{pmp}}$. In between, the probe beam is amplified, except for a small window around $\delta=0$.

As the detuning $\Delta$ is varied from zero, the dressed states no longer have the same steady-state population (see Fig. 4.2). The processes starting from the states with increasing population become more likely and thus, the transmission spectrum becomes less symmetric. Eventually, three-photon amplification, stimulated Rayleigh scattering, and AC-Stark shift are clearly visible. 


\subsection{Two beams interacting with a TLS}

In the previous section, we investigated how a strong pump beam affects a TLS, but we did not yet include any perturbation caused by the probe beam. Here, we present a quantitative description of the interaction of two near resonant beams with a TLS by extending the derivation from subsection 2.1.4 with a second frequency in the excitation field. This case was first theoretically studied by Mollow in 1972 [43]. There, he calculated the transmission spectrum of a weak probe beam, incident on a TLS driven by a strong resonant pump beam. He realized that it is possible in this situation to transfer energy from pump to probe beam, thus amplifying the weaker beam without inverting the TLS population. Later on, these studies were expanded for an off-resonant pump beam, and experiments on ensembles in the gas phase confirmed the theoretical predictions [130, 139]. Even lasers were realized using the stimulated Raman scattering or the three-photon amplification as gain process [140].

In Mollow's approach the probe field is treated as a weak perturbation of the pump-driven TLS. Thus, it is only valid if the probe Rabi frequency $\Omega_{\text {prb }} \ll \Gamma_{1}$. While this is the case for the transmission measurements presented in this work, the condition is not fulfilled for the time-dependent fluorescence measurements, where strong oscillations of the excited state population occur if pump and probe beams are equally strong.

To have a single framework that can describe all experiments presented in this chapter, we follow the approach given in Refs. [84, 132]. Here, the density matrix equations of motion are solved by using a Fourier expansion. By including sufficiently high orders of this expansion, the description of strong probe fields is possible.

The derivation starts with the Hamiltonian from Eq. (2.6), but this time the electric field at the position of the TLS is given by:

$$
\mathbf{E}_{\text {inc }}(\mathcal{O}, t)=\frac{\mathbf{E}_{\mathrm{pmp}}(\mathcal{O})}{2}\left(e^{i \omega_{\mathrm{pmp}} t}+e^{-i \omega_{\mathrm{pmp}} t}\right)+\frac{\mathbf{E}_{\mathrm{prb}}(\mathcal{O})}{2}\left(e^{i \omega_{\mathrm{prb}} t}+e^{-i \omega_{\mathrm{prb}} t}\right) .
$$

The incident electric field has two components: The pump field with frequency $\omega_{\text {pmp }}$ and amplitude $\mathbf{E}_{\text {pmp }}(\mathcal{O})$, and the probe field with frequency $\omega_{\text {prb }}$ and amplitude $\mathbf{E}_{\text {prb }}(\mathcal{O})$, respectively. We omitted the possibility of a relative phase between the two components. This does not affect generality as it is always possible for $\omega_{\text {pmp }} \neq \omega_{\text {prb }}$ to find a time $t_{0}$ at which the two fields are in phase. Unless considering the transient response of the system, 
occurring directly after the light field is switched on, we can shift the time origin to $t_{0}$ and ignore the relative phase [132].

Inserting Eq. (4.6) into Eq. (2.6) yields the new Hamiltonian

$$
\begin{aligned}
\hat{H}=\hbar \omega_{0} \hat{\sigma}^{\dagger} \hat{\sigma} & +\frac{\hbar \Omega_{\mathrm{pmp}}}{2}\left(\hat{\sigma} e^{i \omega_{\mathrm{pmp}} t}+\hat{\sigma} e^{-i \omega_{\mathrm{pmp}} t}+\hat{\sigma}^{\dagger} e^{i \omega_{\mathrm{pmp}} t}+\hat{\sigma}^{\dagger} e^{-i \omega_{\mathrm{pmp}} t}\right) \\
& +\frac{\hbar \Omega_{\mathrm{prb}}}{2}\left(\hat{\sigma} e^{i \omega_{\mathrm{prb}} t}+\hat{\sigma} e^{-i \omega_{\mathrm{prb}} t}+\hat{\sigma}^{\dagger} e^{i \omega_{\mathrm{prb}} t}+\hat{\sigma}^{\dagger} e^{-i \omega_{\mathrm{prb}} t}\right)
\end{aligned}
$$

with pump and probe Rabi frequencies $\Omega_{\mathrm{pmp} / \mathrm{prb}}=-\mathbf{d} \cdot \mathbf{E}_{\mathrm{pmp} / \mathrm{prb}}(\mathcal{O}) / \hbar$. There are two interaction terms in Eq. (4.7), one for each frequency component in the excitation field.

Next, the reference frame is changed to one rotating at $\omega_{\mathrm{pmp}}$. Accordingly, Eq. (4.7) becomes:

$$
\begin{aligned}
\hat{\tilde{H}}= & -\hbar \Delta \hat{\sigma}^{\dagger} \hat{\sigma} \\
& +\frac{\hbar \Omega_{\mathrm{pmp}}}{2}\left(\hat{\sigma}+\hat{\sigma}^{\dagger}+\hat{\sigma} e^{-i 2 \omega_{\mathrm{pmp}} t}+\hat{\sigma}^{\dagger} e^{i 2 \omega_{\mathrm{pmp}} t}\right) \\
& +\frac{\hbar \Omega_{\mathrm{prb}}}{2}\left(\hat{\sigma} e^{i \delta t}+\hat{\sigma}^{\dagger} e^{-i \delta t}+\hat{\sigma} e^{-i\left(\omega_{\mathrm{pmp}}+\omega_{\mathrm{prb}}\right) t}+\hat{\sigma}^{\dagger} e^{i\left(\omega_{\mathrm{pmp}}+\omega_{\mathrm{prb}}\right) t}\right),
\end{aligned}
$$

with $\Delta=\omega_{\mathrm{pmp}}-\omega_{0}$ and $\delta=\omega_{\mathrm{prb}}-\omega_{\mathrm{pmp}}$.

Performing the RWA removes the terms rapidly oscillating at $2 \omega_{\mathrm{pmp}}$ and $\omega_{\mathrm{pmp}}+\omega_{\mathrm{prb}}$. Within this approximation, Eq. (4.8) is given by:

$$
\hat{\tilde{H}}_{\mathrm{RWA}}=-\hbar \Delta \hat{\sigma}^{\dagger} \hat{\sigma}+\frac{\hbar \Omega_{\mathrm{pmp}}}{2}\left(\hat{\sigma}+\hat{\sigma}^{\dagger}\right)+\frac{\hbar \Omega_{\mathrm{prb}}}{2}\left(\hat{\sigma} e^{i \delta t}+\hat{\sigma}^{\dagger} e^{-i \delta t}\right) .
$$

The interaction term still has an explicit time dependence that remains even after the RWA. Using Eq. (4.9) we calculate the equations of motion for the density matrix $\hat{\tilde{\rho}}$ :

$$
\begin{gathered}
\dot{\tilde{\tilde{\rho}}}=\frac{i}{\hbar}\left[\hat{\tilde{\rho}}, \hat{\tilde{H}}_{\mathrm{RWA}}\right] \\
\dot{\tilde{\rho}}_{\mathrm{ee}}=\frac{i}{2}\left(\tilde{\rho}_{\mathrm{eg}}\left(\Omega_{\mathrm{pmp}}+\Omega_{\mathrm{prb}} e^{i \delta t}\right)-\tilde{\rho}_{\mathrm{ge}}\left(\Omega_{\mathrm{pmp}}+\Omega_{\mathrm{prb}} e^{-i \delta t}\right)\right), \\
\dot{\tilde{\rho}}_{\mathrm{eg}}=\frac{i}{2}\left(\tilde{\rho}_{\mathrm{ee}}\left(\Omega_{\mathrm{pmp}}+\Omega_{\mathrm{prb}} e^{-i \delta t}\right)+\tilde{\rho}_{\mathrm{eg}} \hbar \Delta-\tilde{\rho}_{\mathrm{gg}}\left(\Omega_{\mathrm{pmp}}+\Omega_{\mathrm{prb}} e^{-i \delta t}\right)\right), \\
\dot{\tilde{\rho}}_{\mathrm{ge}}=\dot{\tilde{\rho}}_{\mathrm{ge}}^{*}, \quad \dot{\tilde{\rho}}_{\mathrm{gg}}=-\dot{\tilde{\rho}}_{\mathrm{ee}} \cdot
\end{gathered}
$$


Spontaneous decay is included by adding the decay rates $\Gamma_{1}$ and $\Gamma_{2}$ to the equations of motion. Using the Bloch vector $\mathbf{x}=(u, v, w)$, we can express Eqs. (4.10) and (4.11) in form of a single matrix equation [84]:

$$
\begin{gathered}
\dot{\mathbf{x}}=\left(A+\frac{\Omega_{\mathrm{prb}}}{2} e^{i \delta t} B+\frac{\Omega_{\mathrm{prb}}}{2} e^{-i \delta t} B^{*}\right) \mathbf{x}+\mathbf{y}, \\
\text { with } A=\left(\begin{array}{ccc}
-\Gamma_{2} & \Delta & 0 \\
-\Delta & -\Gamma_{2} & -\Omega_{\mathrm{pmp}} \\
0 & \Omega_{\mathrm{pmp}} & -\Gamma_{1}
\end{array}\right), \quad B=\left(\begin{array}{ccc}
0 & 0 & -i \\
0 & 0 & -1 \\
i & 1 & 0
\end{array}\right), \\
\mathbf{y}=\left(\begin{array}{c}
0 \\
0 \\
-\frac{\Gamma_{1}}{2}
\end{array}\right) .
\end{gathered}
$$

This equation can be solved with a Fourier ansatz for the Bloch vector components:

$$
\mathbf{x}=\sum_{n=-\infty}^{\infty} \mathbf{x}_{n} e^{i n \delta t}, \text { with } \mathbf{x}_{n}=\left(\begin{array}{c}
u_{n} \\
v_{n} \\
w_{n}
\end{array}\right)
$$

Inserting Eq. (4.13) into Eq. (4.12) results in a recursive relation for the Fourier coefficients $\mathbf{x}_{n}$

$$
-\mathbf{y} \delta_{n 0}=(A-i n \delta) \mathbf{x}_{n}+B \mathbf{x}_{n-1}+B^{*} \mathbf{x}_{n+1},
$$

with $\delta_{n 0}$ being the Kronecker delta.

With Eq. (4.13) it is possible to define a Fourier series for the timedependent density matrix elements:

$$
\begin{aligned}
& \tilde{\rho}_{\mathrm{ee}}(t)=\sum_{n=-\infty}^{\infty} \tilde{\rho}_{\mathrm{ee}, n} e^{i n \delta t}, \text { with } \rho_{\mathrm{ee}, n}=w_{n}+\frac{1}{2} \delta_{n 0}, \\
& \tilde{\rho}_{\mathrm{ge}}(t)=\sum_{n=-\infty}^{\infty} \tilde{\rho}_{\mathrm{ge}, n} e^{i n \delta t}, \text { with } \tilde{\rho}_{\mathrm{ge}, n}=u_{n}+i v_{n} .
\end{aligned}
$$

In the following two subsections, we use these density matrix elements to calculate the probe beam transmission and the time-dependent fluorescence signal. They are determined by the infinite set of coupled linear equations given in Eq. (4.14). To obtain a numerical solution the set must be truncated [96]. Figure 4.5 depicts the change of the transmission and the fluorescence signal as a function of the truncation order for typical experimental parameters. In case of the transmission signal, the weak probe power leads to a 

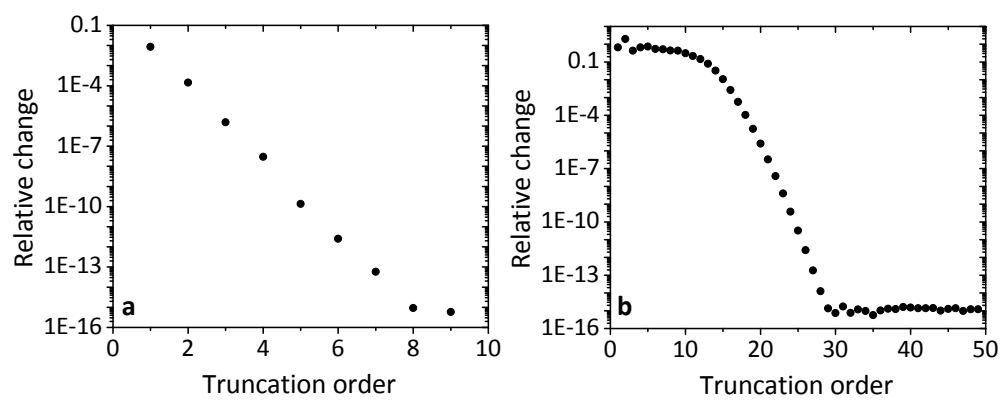

Figure 4.5: Relative change of the transmission signal (a) and the time-dependent fluorescence signal (b) with increasing truncation order. Depicted is the maximum change over the relevant frequency (a) or time scale $(\mathbf{b})$. The calculations use parameters as they occur in the experiment. a, $\Gamma_{2}=\Gamma_{1} / 2, \Omega_{\mathrm{pmp}}=2 \Gamma_{1}, \Omega_{\mathrm{prb}}=0.25 \Gamma_{1}$ and $\Delta=\Gamma_{1} . \mathbf{b} \Gamma_{2}=\Gamma_{1} / 2$, $\Omega_{\mathrm{pmp}}=7 \Gamma_{1}, \Omega_{\mathrm{prb}}=7 \Gamma_{1}, \Delta=0$ and $\delta=\Gamma_{1}$.

quick convergence of the Fourier series. The relative change decreases exponentially with the truncation order until the numerical precision becomes the limiting factor. Already truncation at the second order yields sufficient precision to describe our measurements. To be on the safe side, we include contributions up to the ioth order.

The time-dependent fluorescence signal has a much slower convergence behavior. The main reason is the drastically increased probe Rabi frequency $\Omega_{\mathrm{prb}}=7 \Gamma_{1}$, which is necessary to observe a strong modulation of the excited state population. Until the 1oth order, the relative change is almost constant and only then the result starts converging. Truncation around the zoth order would suffice for an accurate measurement description. But again, to be on the safe side, we include contributions up to the 5oth order.

\subsubsection{Fluorescence}

The formula describing the emission rate of fluorescence photons $R$ is given in Eq. (2.27). Using Eq. (4.15), it is also possible to calculate the timedependent emission rate:

$$
R(t)=\Gamma_{1} \tilde{\rho}_{\mathrm{ee}}(t)=\Gamma_{1} \sum_{n=-\infty}^{n=\infty} \tilde{\rho}_{\mathrm{ee}, n} e^{i n \delta t} .
$$

In the majority of cases, the signal is integrated over a time spanning from $10 \mathrm{~ms}$ to several seconds. This is much longer than even the slowest oscillation period $(2 \pi / \delta)$ of $R(t)$, which is typically a micro-second or less. Thus, contributions of all oscillating components are averaged out and the sum in 

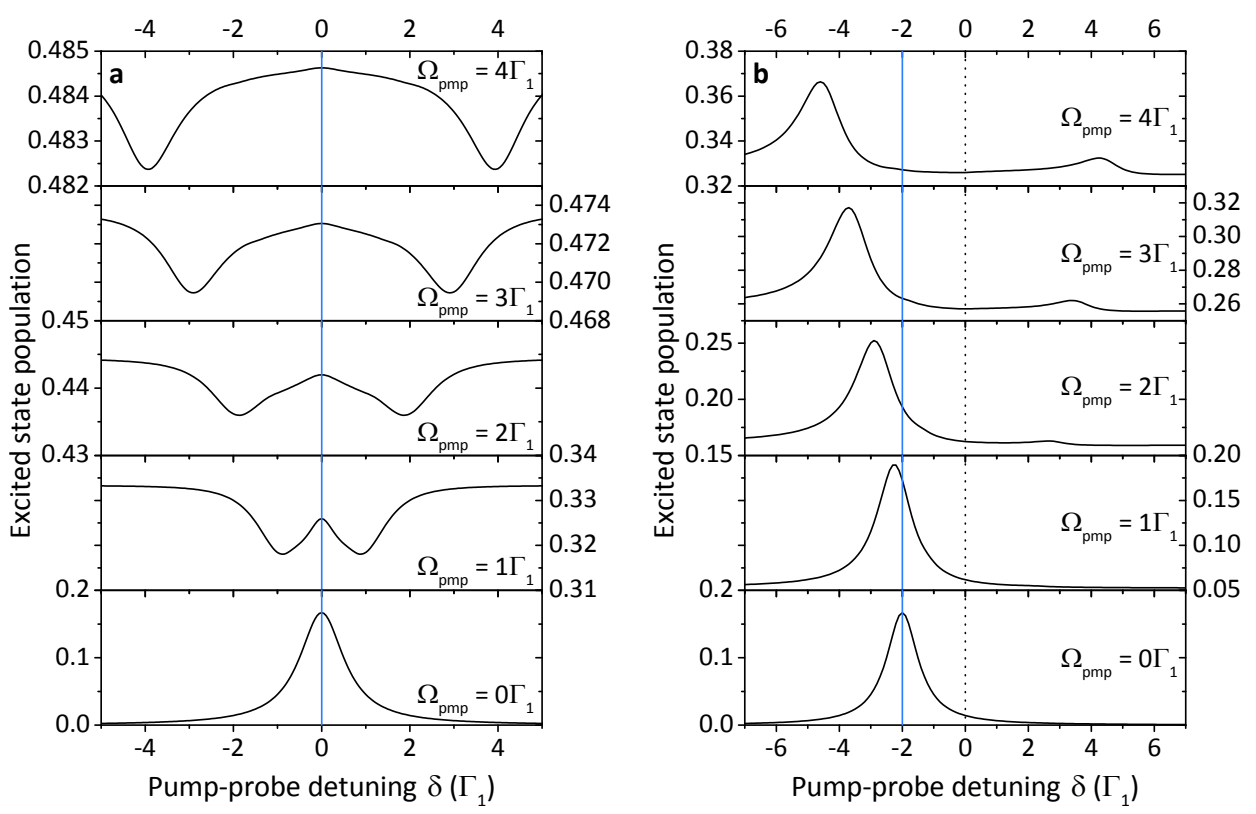

Figure 4.6: Excited state population for different pump Rabi frequencies $\Omega_{\text {pmp }}$ as indicated in the plots. In a the pump beam is on resonance $(\Delta=0)$, while in $\mathbf{b}$ the pump beam is detuned by $\Delta=2 \Gamma_{1}$ from the resonance frequency. The remaining parameters are $\Gamma_{2}=\Gamma_{1} / 2$ and $\Omega_{\text {prb }}=0.5 \Gamma_{1}$. The vertical blue lines indicate the position of the unperturbed resonance frequency and the dotted vertical line shows the pump frequency.

Eq. (4.17) reduces to a single element:

$$
R_{\mathrm{avg}}=\langle R(t)\rangle=\Gamma_{1} \tilde{\rho}_{\mathrm{ee}, 0} .
$$

The impact of the pump beam on the average excited state population, which is directly proportional to the fluorescence signal, can be seen in Fig. 4.6. In a, the pump beam is in resonance with the TLS, causing an increase in the overall fluorescence rate with growing $\Omega_{\mathrm{pmp}}$. Moreover, dips appear in the fluorescence signal for certain pump-probe detunings. This seems at first counterintuitive - the addition of a second driving field lowers the population in the excited state. It can be understood by considering the interference between pump and probe fields. Equation (4.6) describes the incident beam as two fields of fixed amplitude and different frequencies. An equivalent description would be that of a field at a single frequency but with a modulated amplitude [132]. This modulation leads to a periodic change of the strength at which the TLS is driven. Due to saturation, the increase of the 
excited state population $\tilde{\rho}_{\text {ee }}$ during constructive interference of the two fields is less pronounced than the decrease of the excited state population during destructive interference. This results, on average, in a decrease of $\tilde{\rho}_{\text {ee }}$, which is most pronounced if $\delta= \pm \Omega_{\mathrm{pmp}}$ [24].

Two different effects can be observed for an off-resonant pump beam (Fig. $4.6 \mathrm{~b}$ ). For increasing $\Omega_{\text {pmp }}$ the peak of the excited state population is moved away from the pump frequency due to the AC-Stark shift. The second effect is an increase in the excited state population if the resonance condition for the three-photon amplification $\left(\delta=\Omega_{\mathrm{pmp}}^{\prime}\right)$ is fulfilled. In that case, the TLS can be transfered into the excited state via the absorption of two pump photons and the stimulated emission of one probe photon. Indeed, this increase in fluorescence was measured using two laser beams and a single molecule [25].

Besides the time-averaged fluorescence signal, we can also investigate the dynamics of the system at the time scale of the pump-probe beating frequency $\delta$. To that end, all terms of Eq. (4.17) have to be considered. As the excited state population is a real number, its Fourier components are related to each other via

$$
\tilde{\rho}_{\mathrm{ee}, \mathrm{n}}=\tilde{\rho}_{\mathrm{ee},-\mathrm{n}}^{\star} .
$$

Using that relation, we can rewrite Eq. (4.17):

$$
\begin{gathered}
R(t)=\Gamma_{1}\left(\tilde{\rho}_{\mathrm{ee}, \mathrm{o}}+\sum_{n=1}^{\infty} 2\left|\tilde{\rho}_{\mathrm{ee}, \mathrm{n}}\right| \cos \left(n \delta t+\phi_{n}\right)\right) \\
\text { with } \quad \tilde{\rho}_{\mathrm{ee}, \mathrm{n}}=\left|\tilde{\rho}_{\mathrm{ee}, \mathrm{n}}\right| e^{i \phi_{n}} .
\end{gathered}
$$

The time-dependent fluorescence is a periodic signal with components oscillating at different harmonics of $\delta$. The signal is particularly interesting if pump and probe driving strengths are equal. In that case, the resulting field is $100 \%$ amplitude modulated, and thus the driving strength changes significantly within one pump-probe beating period [132].

Figure 4.7 shows the excited state population for $\Omega_{\mathrm{pmp}}=\Omega_{\mathrm{prb}}=5 \Gamma_{1}$. In the time-averaged excited state population (a), one can see multiple resonances. The minima occur for $\delta \approx 1.2 \Omega_{\mathrm{pmp}} / n$, with $n$ being an integer [84]. This phenomenon is called subharmonic Rabi resonances and has been observed for a cloud of atoms [132] and for single molecules [25].

The occurrence of subharmonic resonances is closely related to the evolution of the excited state population during a beating period. Figure $4.7 \mathbf{b}$-d show that evolution for three different values of the pump-probe detuning $\delta$. In plots $\mathbf{b}$ and $\mathbf{c}$ the large detunings of $\delta=4 \Gamma_{1}$ and $\delta=3 \Gamma_{1}$ lead to a fast 

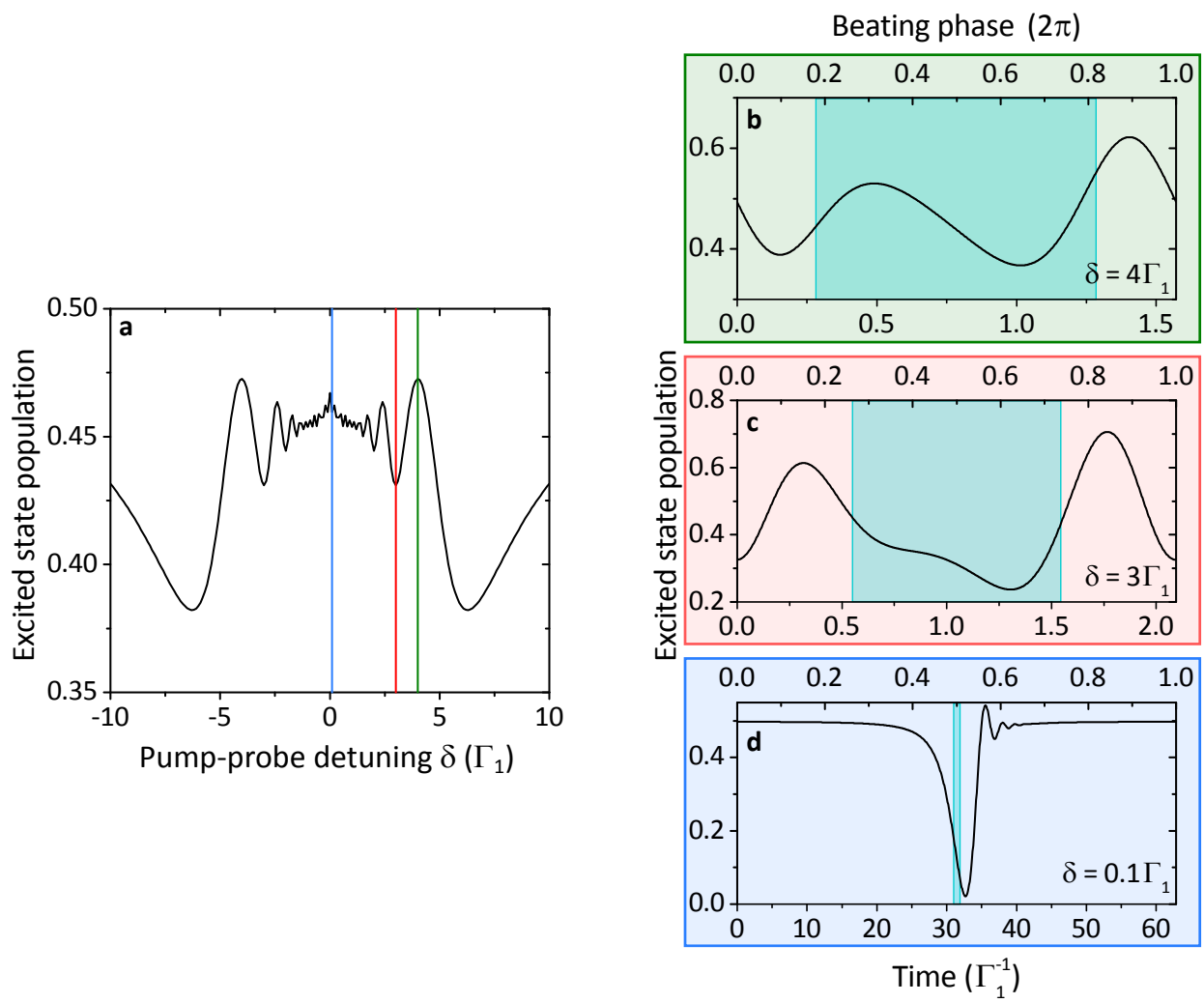

Figure 4.7: Time-averaged and time-dependent excited state population for $\Omega_{\mathrm{pmp}}=\Omega_{\mathrm{prb}}=$ $5 \Gamma_{1}$ and $\Delta=0$. a, Time-averaged excited state population as a function of pump-probe frequency detuning $\delta$. The colored vertical lines indicate the frequency of the probe beam in plots $\mathbf{b}$ - $\mathbf{d}$. $\mathbf{b}$ - $\mathbf{d}$, Time-dependent excited state population. The pump frequency is fixed at $\Delta=0$, while the probe frequency has different values as indicated in each plot. The bottom horizontal axes show time while the upper horizontal axes mark the beating phase $\delta \cdot t$. The turquoise stripes indicate the lifetime.

change of the effective Rabi frequency driving the TLS. Starting from destructive interference of pump and probe fields, the Rabi frequency reaches significant values on a timescale much shorter than the lifetime, causing the excited state population to undergo Rabi oscillations. Depending on the phase of the Rabi oscillation, when pump and probe fields interfere destructively, the average excited state population will show a minimum or a maximum [84]. Figure $4.7 \mathbf{b}$ shows the situation for a maximum of the time-averaged excited state population (green line in a). The Rabi oscillation has a maximum at a beating phase of 0.3 , just before destructive interference of pump and probe beam diminishes the driving strength. At the same beating phase 
the Rabi oscillation shown in $\mathbf{c}$ has a minimum, leading also to a minimum in the time-averaged excited state population (red line in $\mathbf{a}$ ). In part $\mathbf{d}$ the Rabi frequency changes slowly compared to the lifetime of the TLS due to the small pump-probe detuning. Thus, the excited state population is saturated without the occurrence of significant Rabi oscillations.

\subsubsection{Transmission}

The derivation of the transmission signal follows the same approach as in the monochromatic case (see subsection 2.1.6), with the additional complication that two beams are involved. In the experiment, these two beams are not in the same spatial mode that we can separate them in the detection path. Analog to Eq. (2.33), we define two functions $\mathbf{g}_{\text {pmp }}(\mathbf{r})$ and $\mathbf{g}_{\text {prb }}(\mathbf{r})$ to describe the spatial variations of pump and probe fields. Using these, we write the incident field as:

$$
\mathbf{E}_{\text {inc }}^{-}(\mathbf{r}, t)=\mathbf{g}_{\mathrm{pmp}}(\mathbf{r}) E_{\mathrm{pmp}}(\mathcal{O}) e^{i \omega_{\mathrm{pmp}} t}+\mathbf{g}_{\mathrm{prb}}(\mathbf{r}) E_{\mathrm{prb}}(\mathcal{O}) e^{i \omega_{\mathrm{prb}} t},
$$

where $E_{\mathrm{pmp} / \mathrm{prb}}(\mathcal{O})$ is the projection of pump/probe field at the origin, onto the dipole moment $\mathbf{d}$ of the TLS. The transmitted intensity $I_{\text {trans }}$ can be written along the lines of Eq. (2.30), but now the density matrix elements are time-dependent:

$$
\begin{aligned}
I_{\text {trans }} / 2 \epsilon_{0} c= & \\
E_{\mathrm{prb}}^{2}(\mathcal{O}) & \left(\frac{\Omega_{\mathrm{pmp}}^{2}}{\Omega_{\mathrm{prb}}^{2}}\left|\mathbf{g}_{\mathrm{pmp}}(\mathbf{r})\right|^{2}+\left|\mathbf{g}_{\mathrm{prb}}(\mathbf{r})\right|^{2}+\alpha^{2}|\mathbf{f}(\mathbf{r})|^{2} \frac{\Gamma_{1}^{2}}{\Omega_{\mathrm{prb}}^{2}}\left\langle\rho_{\mathrm{ee}}(t)\right\rangle\right. \\
& +2 \frac{\Omega_{\mathrm{pmp}}}{\Omega_{\mathrm{prb}}} \operatorname{Re}\left(\mathbf{g}_{\mathrm{pmp}}^{*}(\mathbf{r}) \mathbf{g}_{\mathrm{prb}}(\mathbf{r})\left\langle e^{i \delta t}\right\rangle\right) \\
& -2 \alpha \frac{\Omega_{\mathrm{pmp}} \Gamma_{1}}{\Omega_{\mathrm{prb}}^{2}} \operatorname{Re}\left(\mathbf{g}_{\mathrm{pmp}}^{*}(\mathbf{r}) \mathbf{f}(\mathbf{r})\left\langle\rho_{\mathrm{ge}}(t) e^{-i \omega_{\mathrm{pmp}} t}\right\rangle\right) \\
& \left.-2 \alpha \frac{\Gamma_{1}}{\Omega_{\mathrm{prb}}} \operatorname{Re}\left(\mathbf{g}_{\mathrm{prb}}^{*}(\mathbf{r}) \mathbf{f}(\mathbf{r})\left\langle\rho_{\mathrm{ge}}(t) e^{-i \omega_{\mathrm{prb}} t}\right\rangle\right)\right)
\end{aligned}
$$

We used the fact that $\Omega_{\mathrm{pmp} / \mathrm{prb}}=-d E_{\mathrm{pmp} / \mathrm{prb}}(\mathcal{O}) / \hbar$, and thus

$$
E_{\mathrm{pmp}}(\mathcal{O}) / E_{\mathrm{prb}}\left(\mathcal{O}_{0}\right)=\Omega_{\mathrm{pmp}} / \Omega_{\mathrm{prb}}
$$


The first three terms describe the intensities of pump field, probe field, and of the light scattered by the molecule, respectively. The three remaining terms account for the interference of pump and probe beams, pump beam and the scattered light, and probe beam and the scattered light.

Except for the first two summands, all terms show an explicit oscillatory time dependence. The dominant oscillation frequency is determined by the pump-probe detuning $\delta$, which is in the range of $1-100 \mathrm{MHz}$ for typical measurements. As the experimental transmission signal is very weak (only around one million photons per second) integration times of milliseconds and longer are required to record the transmitted power with a sufficient signal-to-noise ratio. Using a specialized detection scheme, it is nevertheless possible to resolve such timescales. In section 4.5, we present such a measurement to demonstrate the occurrence of four-wave mixing.

The time averages in Eq. (4.22) can be calculated with the help of Eqs. (4.15) and (4.16):

$$
\begin{aligned}
& \left\langle\rho_{\mathrm{ee}}(t)\right\rangle=\left\langle\sum \tilde{\rho}_{\mathrm{ee}, n} e^{i n \delta t}\right\rangle=\tilde{\rho}_{\mathrm{ee}, 0}, \\
& \left\langle\rho_{\mathrm{ge}}(t) e^{-i \omega_{\mathrm{pmp}} t}\right\rangle=\left\langle\sum \tilde{\rho}_{\mathrm{ge}, n} e^{i n \delta t}\right\rangle=\tilde{\rho}_{\mathrm{ge}, 0}, \\
& \left\langle\rho_{\mathrm{ge}}(t) e^{-i \omega_{\mathrm{prb}} t}\right\rangle=\left\langle\sum \tilde{\rho}_{\mathrm{ge}, n} e^{i(n-1) \delta t}\right\rangle=\tilde{\rho}_{\mathrm{ge}, 1} .
\end{aligned}
$$

Accordingly, Eq. (4.22) simplifies to

$$
\begin{aligned}
I_{\text {trans }} / 2 \epsilon_{0} c=E_{\mathrm{prb}}^{2}(\mathcal{O}) & \left(\frac{\Omega_{\mathrm{pmp}}^{2}}{\Omega_{\mathrm{prb}}^{2}}\left|\mathbf{g}_{\mathrm{pmp}}(\mathbf{r})\right|^{2}+\left|\mathbf{g}_{\mathrm{prb}}(\mathbf{r})\right|^{2}+\alpha^{2}|\mathbf{f}(\mathbf{r})|^{2} \frac{\Gamma_{1}^{2}}{\Omega_{\mathrm{prb}}^{2}} \tilde{\rho}_{\mathrm{ee}, 0}\right. \\
& -2 \alpha \frac{\Omega_{\mathrm{pmp}} \Gamma_{1}}{\Omega_{\mathrm{prb}}^{2}} \operatorname{Re}\left(\mathbf{g}_{\mathrm{pmp}}^{*}(\mathbf{r}) \mathbf{f}(\mathbf{r}) \tilde{\rho}_{\mathrm{ge}, 0}\right) \\
& \left.-2 \alpha \frac{\Gamma_{1}}{\Omega_{\mathrm{prb}}} \operatorname{Re}\left(\mathbf{g}_{\mathrm{prb}}^{*}(\mathbf{r}) \mathbf{f}(\mathbf{r}) \tilde{\rho}_{\mathrm{ge}, 1}\right)\right) .
\end{aligned}
$$

To obtain the transmitted power $P_{\text {trans }}$, Eq. (4.26) has to be integrated over the detection solid angle $A_{\mathrm{det}}$. We are only interested in variations of the probe beam transmission. To that end, we spatially separate the two beams in the experiment, to measure only the probe power. This corresponds to $\mathbf{g}_{\text {pmp }}(\mathbf{r})=0$ over the full detection angle. In practice, this condition is not completely fulfilled. Hence, we include the contribution of residual pump light in the theoretical treatment. 
To calculate the relative change in transmission, it is necessary to define a reference power $P_{0}$. In case of monochromatic excitation, this is the transmitted power of the incident field far from resonance. Now, however, we have to include the interaction of pump beam and TLS, which is occurring even when the probe beam is far detuned. The pump excites the TLS, leading to extinction and the emission of light by the molecule into the detection solid angle. While the contribution of pump beam extinction can be eliminated by perfect spatial separation of the two beams, scattered pump light is always present in the detection solid angle. Taking these effects into account, we write the reference power:

$$
\begin{aligned}
P_{0}= & 2 \epsilon_{0} c E_{\mathrm{prb}}^{2}(\mathcal{O}) \int_{A_{\mathrm{det}}}\left(\frac{\Omega_{\mathrm{pmp}}^{2}}{\Omega_{\mathrm{prb}}^{2}}\left|\mathbf{g}_{\mathrm{pmp}}(\mathbf{r})\right|^{2}+\left|\mathbf{g}_{\mathrm{prb}}(\mathbf{r})\right|^{2}\right. \\
& \left.+\alpha^{2}|\mathbf{f}(\mathbf{r})|^{2} \frac{\Gamma_{1}^{2}}{\Omega_{\mathrm{prb}}^{2}} \tilde{\rho}_{\mathrm{ee}}^{(\mathrm{pmp})}-2 \alpha \frac{\Omega_{\mathrm{pmp}} \Gamma_{1}}{\Omega_{\mathrm{prb}}^{2}} \operatorname{Re}\left(\mathbf{g}_{\mathrm{pmp}}^{*}(\mathbf{r}) \mathbf{f}(\mathbf{r}) \tilde{\rho}_{\mathrm{ge}}^{(\mathrm{pmp})}\right)\right) \mathrm{d} A \\
= & 2 \epsilon_{0} c E_{\mathrm{prb}}^{2}(\mathcal{O}) A_{0} .
\end{aligned}
$$

Here, $\tilde{\rho}_{\mathrm{ge}}^{(\mathrm{pmp})}$ and $\tilde{\rho}_{\mathrm{ee}}^{(\mathrm{pmp})}$ refer to the respective density matrix elements in the absence of the probe beam. Using Eq. (4.27), we can write down the normalized transmission:

$$
\begin{aligned}
T= & \frac{2 \epsilon_{0} c \int_{A_{\mathrm{det}}} I_{\text {trans }} \mathrm{d} A}{P_{0}} \\
= & T_{0}\left(1+T_{\mathrm{pmp}} \frac{\Omega_{\mathrm{pmp}}^{2}}{\Omega_{\mathrm{prb}}^{2}}+\eta_{\mathrm{mol}} \frac{\Gamma_{1}^{2}}{\Omega_{\mathrm{prb}}^{2}} \tilde{\rho}_{\mathrm{ee}, 0}-2 \eta_{\mathrm{pmp}} \frac{\Omega_{\mathrm{pmp}} \Gamma_{1}}{\Omega_{\mathrm{prb}}^{2}} \operatorname{Im}\left(e^{-i \phi_{\mathrm{pmp}}} \tilde{\rho}_{\mathrm{ge}, 0}\right)\right. \\
& \left.-2 \eta_{\mathrm{prb}} \frac{\Gamma_{1}}{\Omega_{\mathrm{prb}}} \operatorname{Im}\left(e^{-i \phi_{\mathrm{prb}}} \tilde{\rho}_{\mathrm{ge}, 1}\right)\right) .
\end{aligned}
$$


The spatial dependence of the fields is included in the following factors:

$$
\begin{gathered}
T_{0}=\frac{\int_{A_{\mathrm{det}}}\left|\mathbf{g}_{\mathrm{prb}}(\mathbf{r})\right|^{2} \mathrm{~d} A}{A_{0}} \\
T_{\mathrm{pmp}}=\frac{\int_{A_{\mathrm{det}}}\left|\mathbf{g}_{\mathrm{pmp}}(\mathbf{r})\right|^{2} \mathrm{~d} A}{\int_{A_{\mathrm{det}}}\left|\mathbf{g}_{\mathrm{prb}}(\mathbf{r})\right|^{2} \mathrm{~d} A} \\
\eta_{\mathrm{mol}}=\alpha^{2} \frac{\int_{A_{\mathrm{det}}}|\mathbf{f}(\mathbf{r})|^{2} \mathrm{~d} A}{\int_{A_{\mathrm{det}}}\left|\mathbf{g}_{\mathrm{prb}}(\mathbf{r})\right|^{2} \mathrm{~d} A} \\
\eta_{\mathrm{pmp}}=i e^{-i \phi_{\mathrm{pmp}}} \alpha \frac{\int_{A_{\mathrm{det}}} \mathbf{g}_{\mathrm{pmp}}^{*}(\mathbf{r}) \mathbf{f}(\mathbf{r}) \mathrm{d} A}{\int_{A_{\mathrm{det}}}\left|\mathbf{g}_{\mathrm{prb}}(\mathbf{r})\right|^{2} \mathrm{~d} A} \\
\eta_{\mathrm{prb}}=i e^{-i \phi_{\mathrm{prb}}} \alpha \frac{\int_{A_{\mathrm{det}}} \mathbf{g}_{\mathrm{prb}}^{*}(\mathbf{r}) \mathbf{f}(\mathbf{r}) \mathrm{d} A}{\int_{A_{\mathrm{det}}}\left|\mathbf{g}_{\mathrm{prb}}(\mathbf{r})\right|^{2} \mathrm{~d} A}
\end{gathered}
$$

Parameters $T_{\mathrm{pmp}}$ and $\eta_{\text {mol }}$ describe the normalized collection efficiencies of the pump beam and the light scattered by the molecule, respectively. $\eta_{\mathrm{pmp} / \mathrm{prb}}$ characterize the normalized mode overlap of the pump and probe beams with the light scattered from the molecule integrated over the detection solid angle. As in the monochromatic case, we define $\eta_{\mathrm{pmp} / \mathrm{prb}}$ with a factor of $i$ to account for the Gouy phase shift and $e^{-i \phi_{\mathrm{pmp} / \mathrm{prb}}}$ to account for any offset from the perfect $\pi / 2$ Gouy phase.

In the ideal case of perfect pump suppression, we get $T_{\mathrm{pmp}}=\eta_{\mathrm{pmp}}=0$. This greatly simplifies Eq. (4.28) and leads to

$$
T_{\text {ideal }}=T_{0}\left(1+\eta_{\mathrm{mol}} \frac{\Gamma_{1}^{2}}{\Omega_{\mathrm{prb}}^{2}} \tilde{\rho}_{\mathrm{ee}, 0}-2 \eta_{\mathrm{prb}} \frac{\Gamma_{1}}{\Omega_{\mathrm{prb}}} \operatorname{Im}\left(e^{i \phi_{\mathrm{prb}}} \tilde{\rho}_{\mathrm{ge}, 1}\right)\right),
$$

which is very similar to the expression for a single beam given in Eq. (2.42).

Using Eq. (4.34), we can calculate the transmission of a weak probe beam in the presence of the strong pump beam. Figure 4.8 a shows this signal in case of a resonant pump beam. For small $\Omega_{\mathrm{pmp}}$, the signal is the usual extinction dip (compare Fig. 2.19). As $\Omega_{\text {pmp }}$ increases, the dip becomes wider and less pronounced due to saturation of the optical transition. At $\Omega_{\mathrm{pmp}} \approx \Gamma_{1}$, new features arise for a pump-probe detuning $\delta \approx \pm \Omega_{\mathrm{pmp}}$. For $\Omega_{\mathrm{pmp}}>1.6 \Gamma_{1}$, these features become peaks with a transmission larger than one, i.e., the 

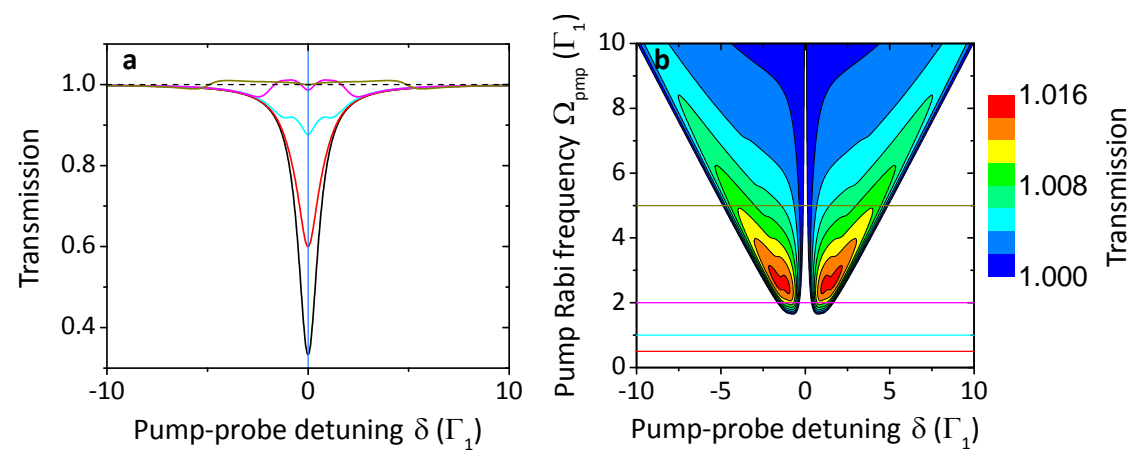

Figure 4.8: Probe beam transmission for varying pump Rabi frequencies for resonant pump $(\delta=0)$. a, Normalized probe beam transmission as a function of the pump-probe detuning $\delta$. The vertical blue line labels the resonance frequency of the TLS. $\mathbf{b}$, Contour plot of the probe beam transmission. The color scale and contour lines are chosen to show only the regime of amplification. The colored horizontal lines indicate the pump Rabi frequency of the respective lines in a. The calculation is done in the case of perfect coupling and complete pump beam suppression, i.e., $\eta_{\mathrm{prb}}=\eta_{\mathrm{mol}}=1$ and $T_{\mathrm{pmp}}=\eta_{\mathrm{pmp}}=0$. The remaining parameters are chosen as in Fig 4.6, i.e., the TLS is lifetime-limited $\left(\Gamma_{2}=\Gamma_{1} / 2\right)$ and $\Omega_{\mathrm{prb}}=0.5 \Gamma_{1}$.

presence of the pumped TLS amplifies the probe beam, even though the TLS does not show population inversion (see Fig. $4.6 \mathrm{a}$ ). The necessary energy for this process is supplied by the pump beam which faces increased absorption when the probe beam is amplified [43]. As $\Omega_{\mathrm{pmp}}$ is increased further, the magnitude of the amplification becomes less but occurs over a larger pumpprobe detuning range. This behavior can be nicely seen in Fig. $4.8 \mathrm{~b}$, which shows the amplification of the probe beam in a $2 \mathrm{D}$ contour plot. The maximum amplification of $1.5 \%$ is achieved for $\Omega_{\text {pmp }} \approx 2.6 \Gamma_{1}$ and $\delta \approx \pm 1.4 \Gamma_{1}$.

The probe beam amplification becomes more pronounced if the pump beam is not exactly on resonance. This situation is depicted in Fig. 4.9. Part a shows the evolution of the probe transmission signal for increasing $\Omega_{\mathrm{pmp}}$ at a fixed detuning of $\Delta=4 \Gamma_{1}$. With growing pump Rabi frequency, the extinction dip moves further away from the pump frequency due to the ACStark effect. An amplification feature emerges at $\delta=\Omega_{\mathrm{pmp}}^{\prime}$, with a maximum amplification of $4.5 \%$ for $\Omega_{\mathrm{pmp}}=8 \Gamma_{1}$.

For each $\Omega_{\mathrm{pmp}}$ there is a specific detuning $\Delta$ that optimizes the probe beam amplification. This can be seen in Fig. 4.9 b: For increasing pump Rabi frequency the optimal detuning grows linearly, while the maximum amplification quickly saturates.

The maximum probe beam amplification is also a function of the probe Rabi frequency. For large $\Omega_{\text {prb }}$, the probe beam significantly perturbs the TLS, leading to a situation where pump as well as probe beam dress the TLS. 

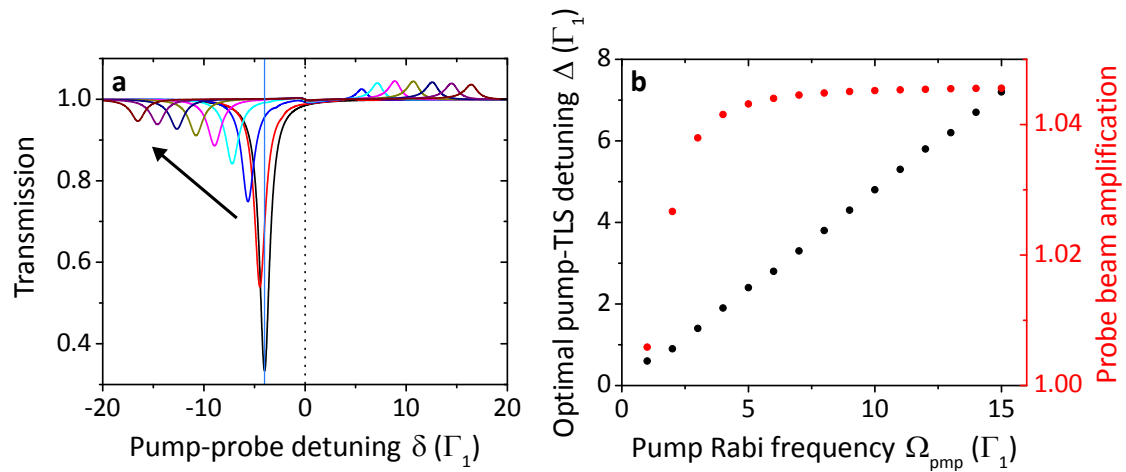

Figure 4.9: Probe beam transmission for an off-resonant pump beam of varying strength. a, Probe beam transmission for a fixed pump detuning $\Delta=4 \Gamma_{1}$. The solid blue and dotted black vertical lines indicate the frequency of the unperturbed resonance and of the pump beam, respectively. The arrow points in the direction of increasing $\Omega_{\mathrm{pmp}}$. b. Maximum achievable amplification and the optimal pump detuning as a function of $\Omega_{\mathrm{pmp}}$. The calculation is done for the ideal case, i.e., $\eta_{\mathrm{prb}}=\eta_{\mathrm{mol}}=1, T_{\mathrm{pmp}}=\eta_{\mathrm{pmp}}=0$, and $\Gamma_{2}=\Gamma_{1} / 2$. The Rabi frequency of the probe beam is fixed at $\Omega_{\text {prb }}=0.5 \Gamma_{1}$, while the pump Rabi frequency is varied in steps of $2 \Gamma_{1}$ from 0 to $16 \Gamma_{1}$.

New processes like the previously mentioned subharmonic Rabi resonances emerge and the probe beam amplification is decreased.

Another situation occurs if $\Omega_{\mathrm{prb}}$ is small. Even in absence of the probe beam, the TLS is excited by the pump beam and scatters light into the detection solid angle. If $\Omega_{\mathrm{prb}} \ll \Gamma_{1}$ the light coming from the TLS dominates the transmitted signal, concealing the amplitude changes of the probe beam. However, it is possible to suppress the contribution of fluorescence by implementing a narrow-band spectral filter. The emission spectrum of the strongly pumped TLS consists mainly of the incoherent Mollow triplet, which has a central peak at $\omega_{\text {pmp }}$ and two sidebands at $\pm \Omega_{\text {pmp }}^{\prime}$ with linewidths of approximately $\Gamma_{1}[59]$. In addition, there is the coherently scattered pump and probe light that maintains the linewidth of the initial fields. If the linewidth of pump and probe fields is much smaller than $\Gamma_{1}$, it is possible to strongly suppress the incoherent scattering in the transmitted signal with a narrow-band filter, which rejects all light not at the probe frequency. If $\omega_{\text {pmp }} \neq \omega_{\text {prb }}$, this spectral filter also removes the coherently scattered pump field.

We include this filter in Eq. (4.34) by replacing $\tilde{\rho}_{\mathrm{ee}, \mathrm{o}}$, which is proportional to the total emission rate, with $\left|\tilde{\rho}_{\mathrm{ge}, 1}\right|^{2}$, which accounts only for coherently scattered light with frequency $\omega_{\text {prb }}$ [71]. In this situation, we have $T_{0}=1$, as the filter also suppresses light at $\omega_{\text {pmp. }}$. Including these changes in Eq. (4.34), 

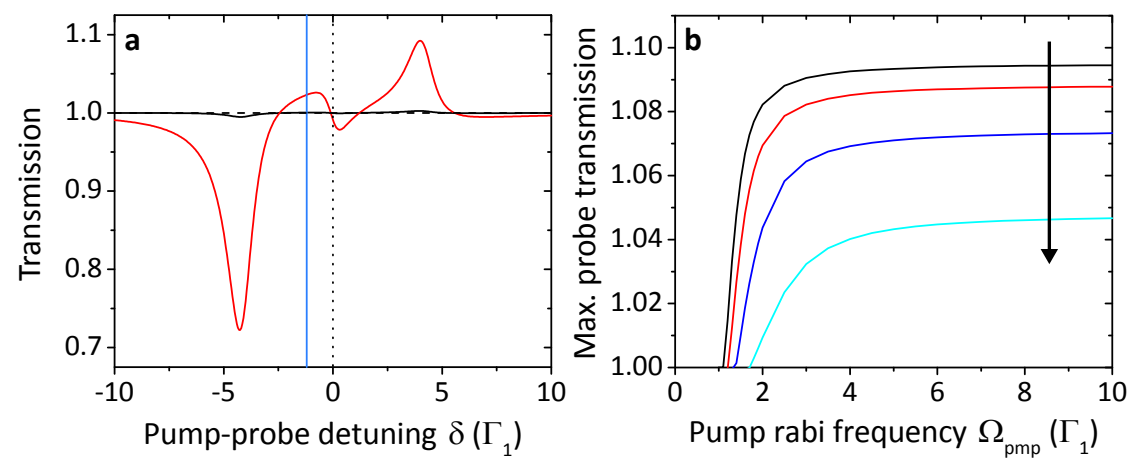

Figure 4.10: a, Probe beam transmission without (black) and with (red) narrow spectral filtering. The vertical dotted black and solid blue lines indicate the pump and TLS resonance frequency, respectively. $\Omega_{\mathrm{prb}}=0.1 \Gamma_{1}, \Omega_{\mathrm{pmp}}=4 \Gamma_{1}$ and $\Delta=1.2 \Gamma_{1} . \mathbf{b}$, Maximum achievable probe beam transmission for $\Omega_{\text {prb }}=0.001,0.5,1$ and $2 \Gamma_{1}$. The arrow points in the direction of increasing $\Omega_{\mathrm{prb}} . \Delta$ is optimized to yield the highest transmission.

we can write the spectrally filtered transmission:

$$
T_{\text {filtered }}=1+\eta_{\mathrm{mol}} \frac{\Gamma_{1}^{2}}{\Omega_{\mathrm{prb}}^{2}}\left|\tilde{\rho}_{\mathrm{ge}, 1}\right|^{2}-2 \eta_{\mathrm{prb}} \frac{\Gamma_{1}}{\Omega_{\mathrm{prb}}} \operatorname{Im}\left(e^{i \phi_{\mathrm{prb}}} \tilde{\rho}_{\mathrm{ge}, 1}\right) .
$$

Figure 4.10 a shows the impact of filtering on the transmitted signal. Without the filter (black curve), the weak probe beam is only a minor part of the transmitted signal. Hence, the features of the transmission spectrum are almost completely obscured by light emitted from the TLS. Once the incoherent background is removed, the relative amplitude changes increase 50 times (red line). In either case, a transmission of one corresponds to vastly different transmitted powers: With spectral filter it is just the amount of detected probe light for $\omega_{\text {prb }}$ far of resonance, while without spectral filter there is additionally the power emitted by the pump beam driven TLS.

However, spectral filtering is only important for very large coupling efficiencies and detection solid angles. In that case, the power necessary to achieve a certain probe Rabi frequency is minimized, while the amount of detected scattered light in the transmission path is maximized. In the experiment, the collection of light emitted by the molecule is negligible compared to the transmitted probe power.

Figure 4.10 $\mathrm{b}$ depicts the maximum achievable probe beam transmission for different probe Rabi frequencies with narrow spectral filtering. For small $\Omega_{\mathrm{prb}}$, the amplification saturates for $\Omega_{\mathrm{pmp}} \geq 3 \Gamma_{1}$ and reaches a maximum value of $9.5 \%$. As $\Omega_{\mathrm{prb}}$ is increased, saturation starts at a higher pump Rabi 


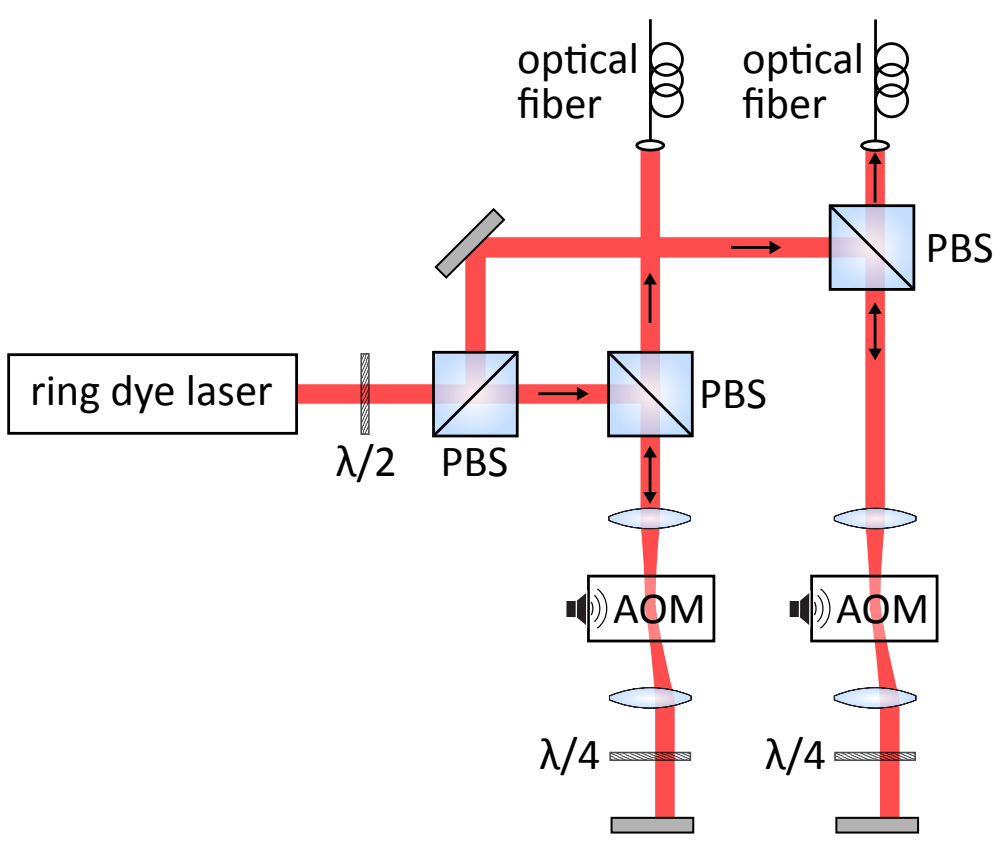

Figure 4.11: Schematic of the double-pass arrangement. The black arrows indicate the propagation direction of the beam. $\lambda / 2$ : half-wave plate, $\lambda / 4$ : quarter-wave plate, PBS: polarizing beam splitter, AOM: acousto-optical modulator. See text for details.

frequency and the maximum amplification is reduced. Hence, it is beneficial for the experiments to operate at low $\Omega_{\mathrm{prb}}$.

\subsection{Transmission measurements}

\subsubsection{Modifications to the setup}

Investigating the interaction of a probe beam with a single strongly pumped molecule requires three major components. First, we need a molecule that is stable, even if driven at high pump rates. Second, the pump and probe laser beams with different frequencies should couple efficiently to the same molecule. And third, we need to separate pump and probe beams after the interaction in order to measure the transmitted power of the latter one.

In the second chapter, we showed that DBATT molecules in a naphthalene matrix fulfill the first requirement as they are very photo-stable. To obtain two laser beams with different frequencies, we use two AOMs (AAOptoelectronic) in a double-pass configuration [84]. Figure 4.11 shows a schematic of the experimental implementation. Light from the ring dye laser is divided 
with a polarizing beam splitter (PBS) into two beams, each going to one AOM. The half-wave plate is used to adjust the intensity in these two arms. The next PBS is oriented such that all light coming from the laser is reflected and then focused into the AOM. The recollimated beam passes a quarter-wave plate and is reflected from a mirror under normal incidence. Hence, the light goes again through the quarter-wave plate and the AOM. Passing the quarter-wave plate twice leads to a polarization rotation of $90^{\circ}$. The light is now transmitted through the PBS and coupled into a single-mode optical fiber.

Light diffracted by an AOM experiences a frequency shift. For the first diffraction order, this shift is given by the frequency of the ultrasonic wave generating the refractive index grating inside the AOM medium. By adjusting the amplitude and frequency of this wave, it is possible to tune the intensity and frequency shift of the diffracted beam, respectively. The double-pass arrangement increases the frequency shift by a factor of two and cancels the change in the diffraction angle when the frequency of the ultrasonic wave is tuned. The latter is necessary to couple the beam efficiently into the singlemode fiber afterwards.

The frequency shift of the pump beam is kept fixed at $400 \mathrm{MHz}$, while the probe beam shift is varied from 315 to $535 \mathrm{MHz}$ using home-built electronics (components from Mini-Circuits) leading to a possible pump-probe detuning $\delta / 2 \pi$ of -85 to $135 \mathrm{MHz}$. The range is limited by the decreasing diffraction efficiency of the AOM away from its center frequency and imperfect alignment of the double-pass arrangement causing residual angle changes of the first diffraction order. The intensity of each diffracted beam is kept constant with a PID feedback loop.

Figure 4.12 displays a schematic of the optical setup. The probe beam follows the normal excitation path, described in subsection 2.2.1. The pump beam however, is guided along a different way into the cryostat. After leaving the optical fiber, a high NA objective focuses the beam into the back focal plane of the second lens of the telecentric system. Objective and lens form a telescope leading to a small pump beam diameter. A polarizer ensures perfect linear polarization and a beam splitter reflects part of the beam onto a photodiode to obtain the reference signal for the intensity stabilization. A subsequent neutral density filter adjusts the pump power to the required level. Using a 50:50 pellicle beam splitter, the pump beam is merged with the probe beam and directed into the cryostat. The advantage of a pellicle beam splitter is the absence of a displacement of the transmitted probe beam, when the pellicle is added or removed. However, its reflectivity is highly polarization dependent. Thus, the half-wave plate adjusting the pump beam polarization 


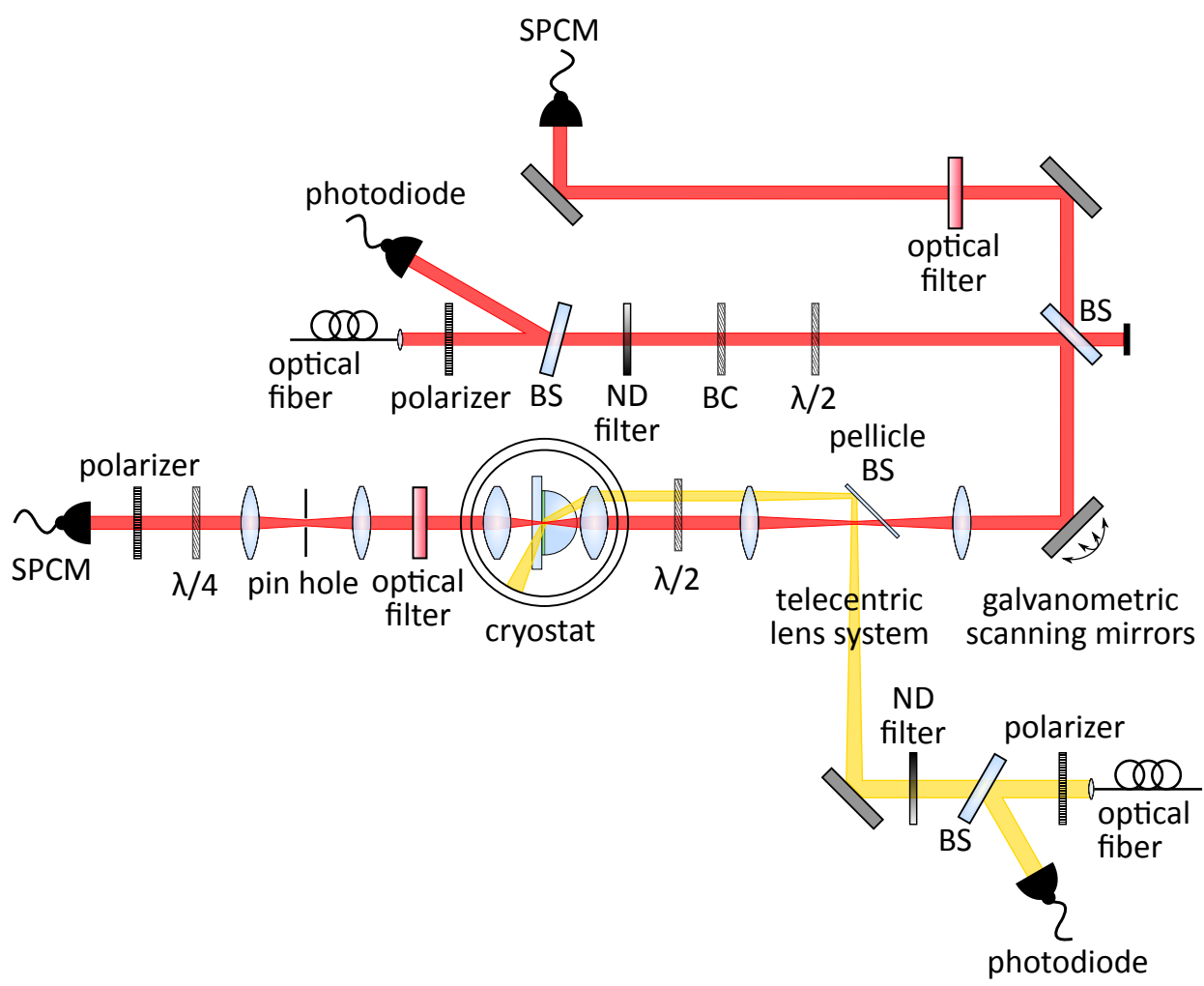

Figure 4.12: Optical paths of pump (yellow) and probe (red) beam. See text for a detailed explanation. BS: beam splitter, ND filter: neutral density filter, BC: Berek compensator, $\lambda / 2$ : half-wave plate, $\lambda / 4$ : quarter-wave plate, SPCM: single photon counting module.

is placed behind the pellicle. Any changes induced in the probe beam polarization are compensated with the Berek compensator and the probe beam half-wave plate.

In contrast to the probe, the pump beam is aligned close to the edge of the optics, which ensures that the aspheric lens inside the cryostat focuses the pump onto the sample under a high incidence angle. The large refractive index of the SIL leads to further diffraction of the pump beam out of the collection angle covered by the second aspheric lens (see Fig. $4.13 \mathrm{a}$ ). This technique achieves spatial separation of the pump and probe beams. Unfortunately, this also compromises the pump beam coupling efficiency.

Owing to its imperfect focusing, the pump light illuminates an extended sample region and faces scattering at crystal defects leading to residual amounts of pump light in the probe beam behind the cryostat. To further reduce the amount of pump light in the transmitted signal, it is focused onto 
a

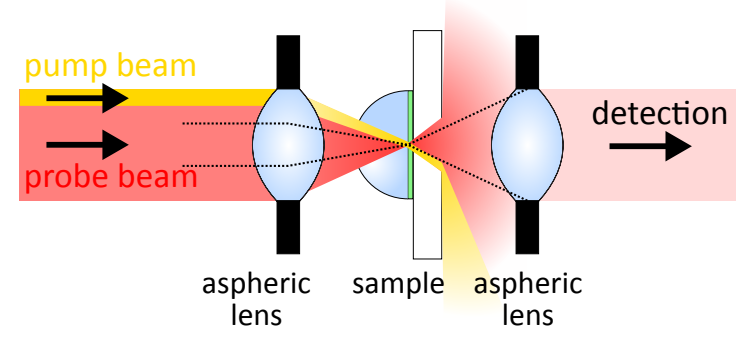

b

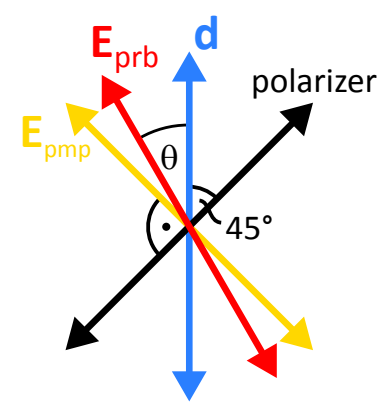

Figure 4.13: a, Spatial filtering of the pump beam. The dashed line indicates the collection solid angle. b, Polarization of pump and probe beam, orientation of the molecular dipole moment $\mathbf{d}$ and the polarizer. $\theta$ is the angle between probe field and dipole moment.

a pinhole blocking light not coming from the probe beam focal volume. Additionally, the pump light polarization is set at $45^{\circ}$ angle to the dipole orientation such that it can be filtered out by a polarizer at $-45^{\circ}$ angle (see Fig. 4.13 b). The quarter-wave plate in front of the polarizer is used to compensate the ellipticity of the pump beam, which can be introduced by the optical elements inside the cryostat. Red-shifted fluorescence in the transmitted signal is blocked with an optical band-pass filter (Semrock).

The contribution of the residual pump light is quantified by $T_{\mathrm{pmp}}$ as defined in Eq. (4.30). It corresponds to the ratio of pump to probe power in the transmitted signal if both beams drive the molecule with the same Rabi frequency, i.e., $\Omega_{\mathrm{pmp}}=\Omega_{\mathrm{prb}}$. We typically achieve a value of $T_{\mathrm{pmp}} \approx 1 / 80$.

The polarizer can furthermore be used to increase the visibility of the interference between probe and light scattered off the molecule, thus emphasizing the nonlinear effects. In analogy, the visibility of an interference pattern behind a double slit is maximized if the intensities originating from both slits are equal. Figure 4.14 a shows this effect exemplary for the probe beam extinction. If the probe beam polarization is parallel to the dipole moment of the molecule, the fluorescence signal is maximized and the polarizer attenuates both light fields by the same amount, leading to an extinction signal of $5 \%$. If the probe beam polarization angle $\theta$ is increased, the amount of light scattered by the molecule is reduced due to a decreasing coupling efficiency. However, as Fig. 4.14 b shows, the amount of probe light transmitted through the polarizer (blue line) is decreased far more, leading to an increase in the ratio of molecule to probe light after the polarizer (red line). As the probe light typically dominates in the transmission signal, this makes both intensities more equal and thus increases the visibility of the extinction signal up to $18 \%$ at $\theta=45^{\circ}$. However, as at the same time the absolute transmitted power 

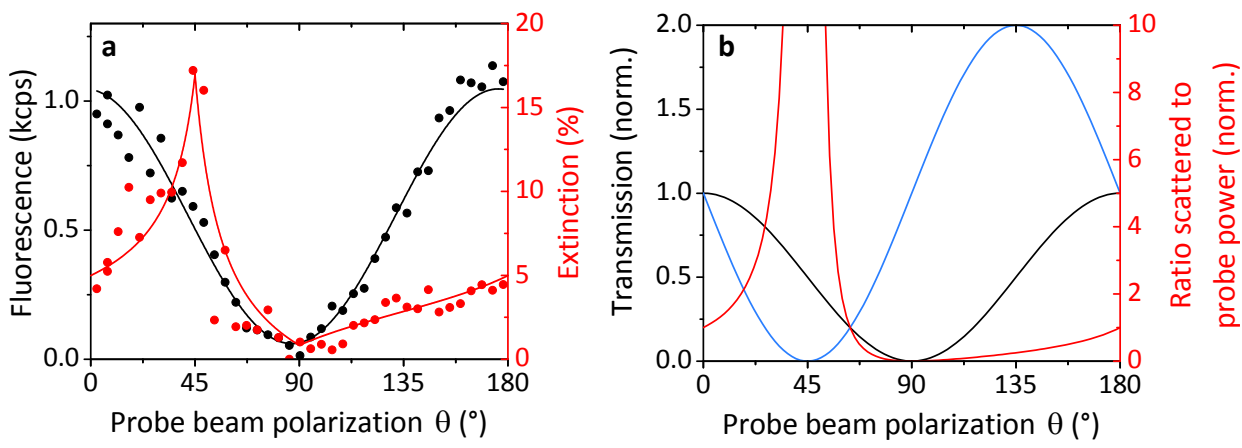

Figure 4.14: Effect of the probe beam polarization on the transmitted power and the scattered light. a, Fluorescence (black squares) and extinction (red circles, right hand y-axis) as a function of the probe beam polarization angle $\theta$. The solid lines are fits of the theoretical model to the data. $\mathbf{b}$, Amount of probe light (blue) and light emitted by the molecule (black) that is transmitted through the polarizer. The red curve shows the change of their ratio and is projected on the right hand axis. All three curves are normalized to 1 for $\theta=0$.

is decreased, there is a trade off between size of the effect and necessary integration time.

If the probe beam polarization angle is increased further, the ratio of the scattered to transmitted probe power reduces and so does the extinction signal. After a minimum at $\theta=90^{\circ}$, where there is no coupling between probe light and molecule, the extinction signal slowly recovers again.

For the experiment, it is favorable to choose a probe beam polarization angle $\theta$ slightly below $45^{\circ}$, which leads to an extinction signal of around $10 \%$, while still giving a large enough count rate to measure a signal change of $0.1 \%$ within one second integration time.

\subsubsection{Measurement and evaluation sequence}

In a typical experiment, probe beam transmission spectra are recorded for various settings of the pump Rabi frequency $\Omega_{\mathrm{pmp}}$ and the pump-molecule detuning $\Delta$. First, the pump Rabi frequency is determined from the power broadening of the fluorescence excitation spectrum, which is directly related to $\Omega_{\text {pmp }}$ via Eq. (2.28).

In a next step, the pump beam is scanned across the molecule to determine its center frequency so that the pump-molecule detuning can be set exactly. The probe beam transmission is very sensitive even to small changes of $\Delta$, which can occur due to laser frequency drifts or spectral diffusion of the molecular resonance. In order to account for these variations, the probe 


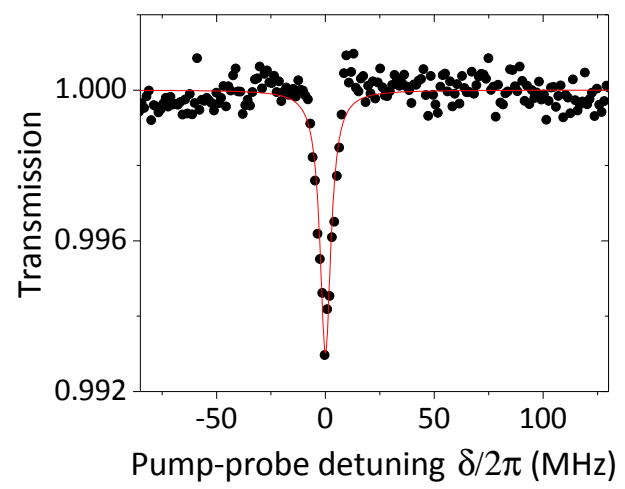

Figure 4.15: Probe beam transmission signal if no molecule is present. Interference of probe and residual pump light leads to a dip around $\delta=0$. The red line is a Lorentz curve fitted to the data.

beam is first tuned within one second across the resonance and $\Delta$ is readjusted afterwards. These two steps are repeated several hundred times until an integration time of typically one second per frequency pixel is obtained.

The resulting transmission signal is corrected for residual probe intensity fluctuations, by normalizing it with the reference photodiode signal, which is also used for intensity stabilization. Afterwards, the expected probe transmission, described by Eq. (4.28), is fitted to the measured data. To reduce the number of parameters, we make the following simplifications: We assume that pump and probe beams have the same spatial mode over the detection angle, but the pump field strength is reduced by a factor $\sqrt{T_{\mathrm{pmp}}}$ :

$$
\mathbf{g}_{\mathrm{pmp}}(\mathbf{r}) \approx \mathbf{g}_{\mathrm{prb}}(\mathbf{r}) \sqrt{T_{\mathrm{pmp}}} .
$$

This approximation is justified, since the transmitted light is spatially filtered using a pinhole. Thus, residual pump light that remains after the pinhole should be in the same mode as the probe beam. From Eq. (4.36) it also follows that the deviation from the ideal $\pi / 2$ Gouy phase shift is equal for pump and probe, i.e., $\phi_{\mathrm{pmp}}=\phi_{\mathrm{prb}}$. Accordingly, we obtain the relation:

$$
\eta_{\mathrm{pmp}}=\sqrt{T_{\mathrm{pmp}}} \eta_{\mathrm{prb}}
$$

Additionally, we identify $\eta_{\mathrm{mol}}=\eta_{\mathrm{prb}}^{2}$, which we already used in the single beam case, treated in subsection 2.1.6.

Figure 4.15 shows the destructive interference of pump and probe beams occurring on the detector around $\delta \approx 0$ if no molecule is present. The dip is 

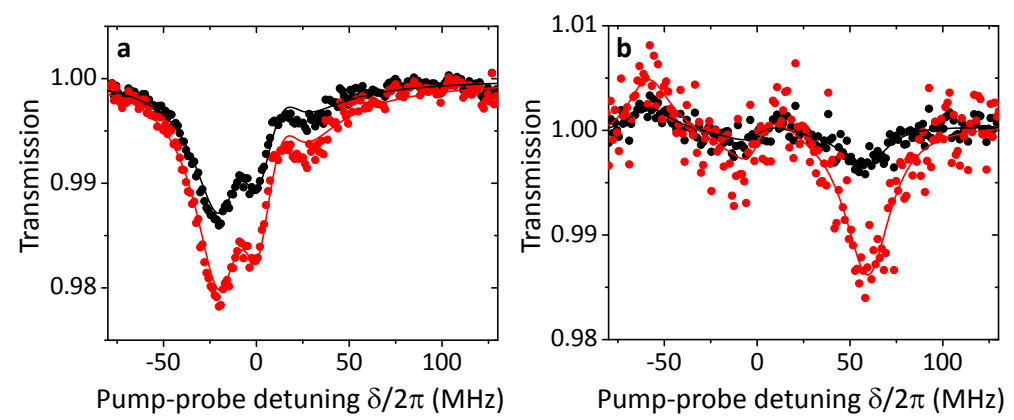

Figure 4.16: Probe beam transmission with (red) and without correction (black) for residual pump light. The solid lines are a fit to the data. a, weak pump beam case with $\Omega_{\text {pmp }} \approx 4 \Omega_{\text {prb }}$ and $\Delta=5 \mathrm{MHz}$. b, strong pump beam case with $\Omega_{\mathrm{pmp}} \approx 13 \Omega_{\mathrm{prb}}$ and $\Delta=-14 \mathrm{MHz}$.

well described by a Lorentzian curve with a FWHM of $6 \mathrm{MHz}$, corresponding to roughly twice the laser linewidth. We divide the probe beam transmission signal by the normalized Lorentzian curve to remove this artifact.

In total, this leaves us with the following parameters:

- The natural linewidth $\Gamma_{1}$ and the dephasing rate $\Gamma_{2}$. While the natural linewidth is known from literature, the dephasing rate can determined via fluorescence excitation scans at weak power. Both are fixed values for the fit.

- The relative suppression of the pump beam $T_{\mathrm{pmp}}$. This is determined by measuring the transmitted pump and probe powers at a given Rabi frequency and is kept at a fixed value.

- Pump and probe Rabi frequencies $\Omega_{\mathrm{pmp} / \mathrm{prb}}$ and the pump-molecule detuning $\Delta$ are initially set to the experimental target values, but they are then treated as free parameters to allow for a small offset.

- The mode overlap of probe and molecular light $\eta_{\mathrm{prb}}$ and the deviation from the ideal Gouy phase shift $\phi_{\text {prb }}$ are as well free fit parameters.

- The magnitude of the correction for the destructive pump-probe interference shown in Fig. 4.15.

Using these six free parameters, it is possible to fit the theoretical model to the data.

As mentioned earlier, we typically achieve a pump beam suppression $T_{\mathrm{pmp}} \approx 1 / 80$. However, we perform measurements where $\Omega_{\mathrm{pmp}}$ is more than ten times larger than $\Omega_{\text {probe }}$. As incident and transmitted power depend 
quadratically on the Rabi frequency, this means that the ratio of the detected pump to probe power can be $1: 1$ or even worse. This results in a significant influence of the residual pump light on the measured signal. To remove this unwanted contribution, we first fit the theory curve of Eq. (4.28), which accounts also for transmitted pump light. Using the optimized parameters, we calculate the changes caused by the residual pump light and correct the signal by subtracting the pump beam contribution.

Figure 4.16 shows the impact of that correction for relatively low (a) and high pump power (b). In both cases the shape is barely affected, but the magnitude of the signal variation increases.

\subsubsection{Experimental results}

In order for the experimental results to be comparable, all measurements were performed with the same DBATT molecule. It showed a linewidth of $\Gamma_{1} / 2 \pi=20 \mathrm{MHz}$ and exceptionally high photo-stability, which was required as the measurement time for a single transmission spectrum easily exceeded one hour. This made the mediocre coupling efficiency of only $3 \%$ acceptable.

The most relevant parameters that determine the transmission spectrum are the pump-molecule detuning $\Delta$ and the pump Rabi frequency $\Omega_{\mathrm{pmp}}$. We present first the evaluation of the transmission signal with increasing $\Omega_{\mathrm{pmp}}$ for two fixed values of $\Delta$. Afterwards, the pump Rabi frequency is kept fixed, while the pump-molecule detuning is varied. All measurements are corrected for the pump-probe interference around $\delta=0$ and for residual pump light, as described in the previous subsection. The probe beam power is varied between $\Omega_{\text {prb }} / 2 \pi=2-6 \mathrm{MHz}$, i.e., it is always much smaller than $\Gamma_{1}$.

Figure 4.17 displays the evolution of the probe beam transmission spectrum, as the Rabi frequency of the resonant pump beam is increased from 0 - $57 \mathrm{MHz}$. Without the pump beam (a), we observe the usual extinction dip. As the pump beam is turned on, the extinction dip becomes shallower due to saturation of the $S_{0} \rightarrow S_{1}$ transition. In addition, sidebands emerge already for $\Omega_{\mathrm{pmp}}<\Gamma_{1}$ (b), forming the precursor of the Mollow absorption spectrum. As $\Omega_{\mathrm{pmp}}$ is increased further, two dispersive features arise (c) with a transmission equal to 1 at a pump-probe detuning $\delta \approx \pm \Omega_{\mathrm{pmp}}$. Moreover, the signal shows a transmission exceeding 1 , corresponding to amplification of the weak probe beam. As explained in section 4.1, this demonstrates energy transfer from the pump to the probe beam, mediated by the molecule.

In part $\mathbf{d}$, the dispersive wings become even wider, causing probe beam amplification over a frequency range of roughly $50 \mathrm{MHz}$ with a peak value of 
$0.5 \%$. The asymmetry of the transmission signal is caused by a small pumpmolecule detuning of just $-2 \mathrm{MHz}$, emphasizing the importance of maintaining a stable pump-molecule detuning throughout the measurement. At such large pump Rabi frequencies, the residually collected pump light starts to outweigh the probe beam power in the transmission signal. Furthermore, theory predicts weaker modulations of the probe beam transmission (see Fig. 4.8) for a resonant pump beam. Thus, we we did not continue these measurements beyond $\Omega_{\mathrm{pmp}} / 2 \pi \approx 60 \mathrm{MHz}$, corresponding to a saturation parameter of $S=16$.

Figure 4.18 shows the evolution of the probe beam transmission with increasing $\Omega_{\text {pmp }}$, in case of a pump-molecule detuning of $\Delta / 2 \pi \approx 6 \mathrm{MHz}$. With the pump beam turned on, the frequency of minimum transmission is shifted due to the AC-Stark effect. As before, increasing saturation of the molecule decreases the size of the extinction dip. At the same time, a dispersive feature emerges around $\delta \approx 0$, as can be nicely seen in the green region of part $\mathbf{c}$. It is caused by stimulated Rayleigh scattering, which leads to an energy transfer between pump and probe beam. In the blue region of part $\mathbf{c}$, the probe beam is amplified via a three-photon transition by up to $0.3 \%$.

The measurement at the highest Rabi frequency $(\mathbf{d})$ is again at the edge of the feasible parameter space. At such high pump powers, the contribution of the residual pump light is almost four times stronger than the collected probe light. The signal is furthermore, strongly affected by a deviation from the ideal Gouy phase shift, which significantly distorts the probe beam transmission, e.g., it is removing the three-photon amplification signal around $\delta / 2 \pi=50 \mathrm{MHz}$. The combination of these two effects renders measurements at even higher pump Rabi frequencies impossible. Nevertheless, the data is perfectly reproduced by the theoretical model.

We now focus on the situation, where $\Delta$ is varied while $\Omega_{\mathrm{pmp}}$ is kept constant. In Fig. 4.19, the pump Rabi frequency $\Omega_{\text {pmp }} / 2 \pi \approx 17 \mathrm{MHz}$. As indicated by the blue line, which marks the transition frequency of the unperturbed molecule, the pump-molecule detuning $\Delta / 2 \pi$ is varied from $10 \mathrm{MHz}$ in a to $-5 \mathrm{MHz}$ in $\mathbf{d}$. Each step corresponds just to a quarter of the transition linewidth and nevertheless changes the probe transmission spectrum significantly. In $\mathbf{a}$ and $\mathbf{b}$, the AC-Stark effect causes a shift of the minimum transmission to lower frequencies, while in part $\mathbf{d}$ the changing sign of $\Delta$ leads to a shift to higher frequencies.

Figure 4.20 shows a similar evolution of the transmission signal, but this time at a larger pump Rabi frequency of $\Omega_{\mathrm{pmp}} / 2 \pi \approx 37 \mathrm{MHz}$ and over a slightly extended pump-molecule detuning range of $13 \mathrm{MHz}$ in a to $-10 \mathrm{MHz}$ 


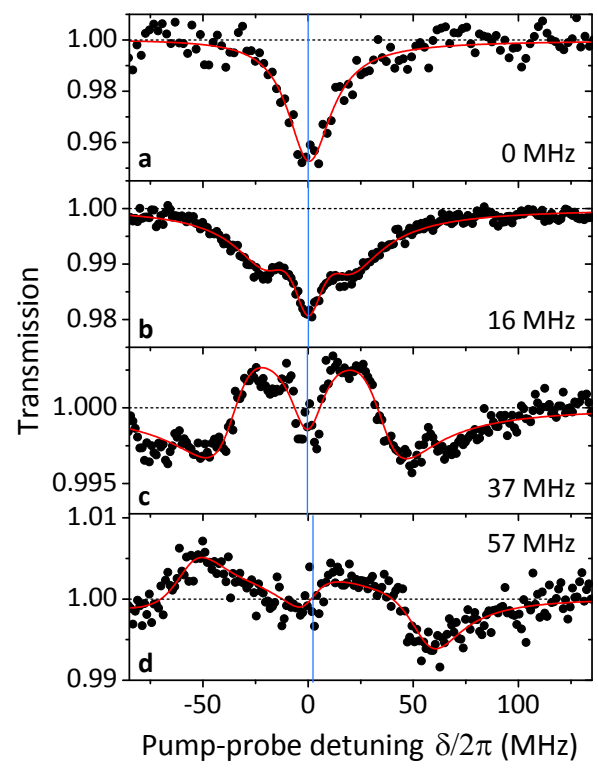

Figure 4.17: Probe beam transmission with pump beam on resonance. The pump beam Rabi frequency is increased from $\mathbf{a}-\mathbf{d}$ with the exact values of $\Omega_{\mathrm{pmp}} / 2 \pi$ noted in each plot. The solid red lines are fits to the data. The vertical blue line denotes the transition frequency of the unperturbed molecule.

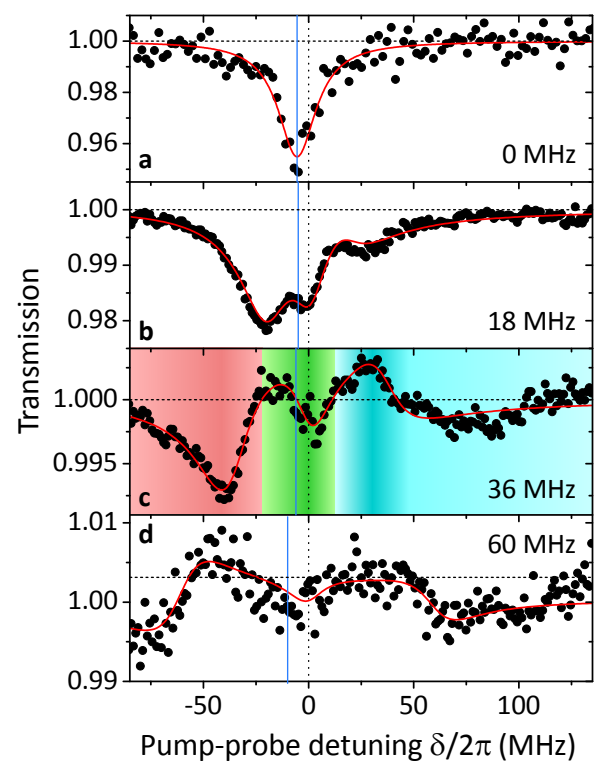

Figure 4.18: Probe beam transmission with a pump-molecule detuning of $\Delta / 2 \pi \approx$ $6 \mathrm{MHz}$. The pump beam Rabi frequency $\Omega_{\mathrm{pmp}} / 2 \pi$ is increased from a - $\mathbf{d}$ with the exact value noted in each plot. The solid red lines are fits to the data. The vertical solid blue and dotted black lines denote the transition frequency of the unperturbed molecule and the pump frequency, respectively. The colored regions in $\mathbf{c}$ indicate the regimes of AC-Stark shift (red), stimulated Rayleigh scattering (green) and threephoton amplification (blue). 


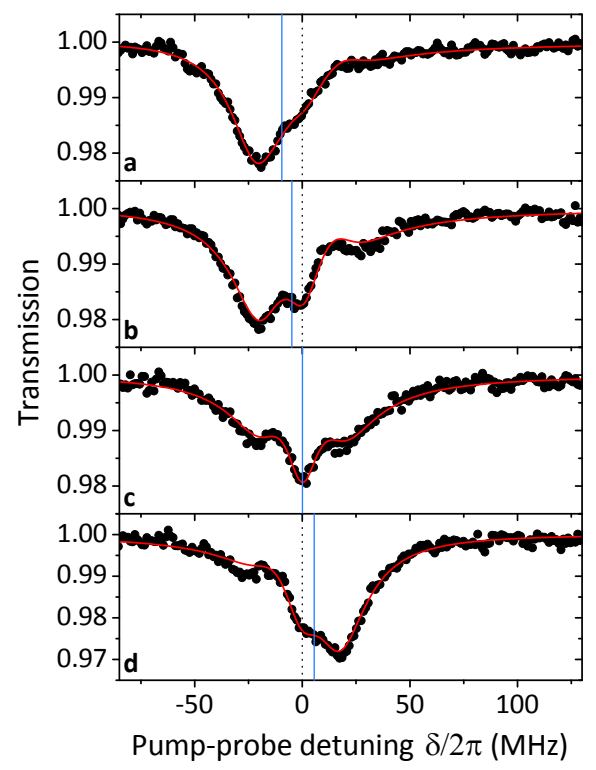

Figure 4.19: Probe beam transmission with varying pump-molecule detuning $\Delta$, for $\Omega_{\text {pmp }} / 2 \pi \approx 17 \mathrm{MHz} . \Delta$ is decreased from a - d. The solid red lines are fits to the data. The vertical solid blue and dotted black lines denote the transition frequency of the unperturbed molecule and the pump frequency, respectively.

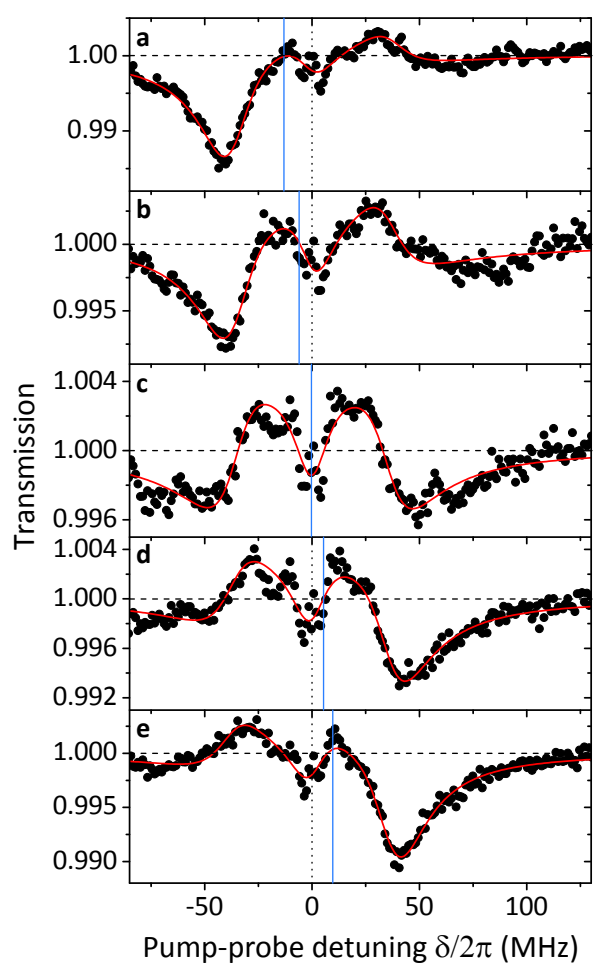

Figure 4.20: Probe beam transmission with varying pump-molecule detuning $\Delta$, for $\Omega_{\mathrm{pmp}} / 2 \pi \approx 37 \mathrm{MHz} . \Delta$ is decreased from a - e. The solid red lines are fits to the data. The vertical solid blue and dotted black lines denote the transition frequency of the unperturbed molecule and the the pump beam frequency, respectively. 
in e. Besides the changing AC-Stark shift, here also stimulated Rayleigh scattering and three-photon amplification are affected.

Similar to the AC-Stark effect, stimulated Rayleigh scattering changes its behavior with the sign of the pump-molecule detuning. If $\Delta>0$ ( $\mathbf{a}$ and $\mathbf{b}$ ), the probe beam is amplified via this process in a region with $\delta<0$. As the pump-molecule detuning becomes negative ( $\mathbf{d}$ and $\mathbf{e}$ ), the dispersive shape of the stimulated Rayleigh scattering is flipped and the probe beam is now amplified at positive pump-probe detuning.

The three-photon amplification is affected in two ways by the pumpmolecule detuning. From a to $\mathbf{b}$, the amplification is shifted to a slightly lower frequency, and, at the same time, the maximum amplification increases marginally from $0.25 \%$ to $0.28 \%$. This is in perfect agreement with the prediction of a particular pump-molecule detuning which optimizes the probe beam amplification, as shown earlier in Fig. 4.9. The same behavior is repeated for negative pump-molecule detuning ( $\mathbf{d}$ and $\mathbf{e}$ ). Amplification occurs now at a negative pump-probe detuning and has a maximum value of $0.30 \%$ in $\mathbf{d}$ and slightly smaller value of $0.26 \%$ in $\mathbf{e}$.

Figures 4.17 to 4.20 demonstrate how the pump beam controls the probe transmission spectrum via a nonlinear interaction with a single molecule. In a linear regime, all plots would display the same curve, as the interaction of probe and molecule would be not affected by the pump beam. Instead, the nonlinear response of a single molecule leads to all features predicted by the dressed state model - AC-Stark shift, stimulated Rayleigh scattering and three-photon amplification. Over a wide parameter range, the measured data are well described by our theoretical model.

\subsection{Few-photon optical switching}

The measurements show that the interaction of two light beams does not require high optical power or bulk nonlinear crystals. Two faint beams tightly focused onto a single molecule can already yield significant effects. The key component is our high coupling efficiency, which becomes evident when we compare our results to previous ensemble measurements. In case of the experiments presented in Ref. [139], around $10^{10}$ atoms were necessary to achieve 38 -fold amplification of the probe beam using the same nonlinear process. This corresponds to an amplification of approximately $4 \times 10^{-10}$ per atom. We report a value of $0.3 \%$ using a single molecule, which is about seven 
orders of magnitude larger. This dramatic increase is enabled by tight focusing, a strong lifetime-limited transition, and incident fields with frequencies close to the molecular resonance.

This result paves the way towards cavity-free nonlinear optics with single emitters and weak light fields of only few photons per lifetime. Even though the molecule used in this chapter shows only a coupling efficiency of $3 \%$, which is further reduced to $2 \%$ taking the probe beam polarization into account, this still means that just 1.6 probe photons per lifetime are present during the measurements of Fig. 4.17 to Fig. 4.20.

The spatial separation of pump and probe beams requires a different optical path for both beams. Owing to its small beam diameter, the coupling efficiency of the pump beam is roughly ten times lower. Thus, around 960 pump photons per lifetime were necessary to observe the probe beam transmission spectrum of Fig. $4.18 \mathrm{c}$. While the spatial separation is a convenient method, it is not the only way to differentiate pump and probe beam in the transmission signal. Another feasible, but experimentally more challenging, technique is the use of a narrow-band optical cavity to spectrally separate pump and probe beam. In that case, pump and probe can share the same spatial mode and can, hence, be coupled equally efficiently to the single molecule. If we, moreover, assume a coupling efficiency of $5 \%$, which is routinely achieved, then the number of pump photons reduces from 960 to 32 photons per lifetime. By further increasing the NA of the focusing optics and using a directional dipole mode to excite the molecule [66], it should be possible to increase the coupling efficiency to $\sim 50 \%$, which is then only limited by the Debye-Waller and Franck-Condon factors of the molecule. At this coupling efficiency, the number of required pump photons would be reduced to as few as 3 photons per lifetime.

A possible application of such a strong nonlinearity, would be the switching of a weak beam with few photons, which is an important technology for optical quantum and classical information processing [26]. Indeed, our system enables such an interaction [42]. The green curve in Fig. 4.21 a shows the probe beam transmission in the absence of a pump beam. For this measurement, the mode overlap of the light scattered by the molecule and the probe beam on the detector is increased by using a single-mode fiber to collect the transmitted light. This leads to an extinction dip of 30\%. Furthermore, the precise spatial selection of the optical fiber enhances the relative pump beam suppression to more than 200. These improvements come at the expense of a reduced count rate, as all light not matching the mode of the fiber is not collected. 

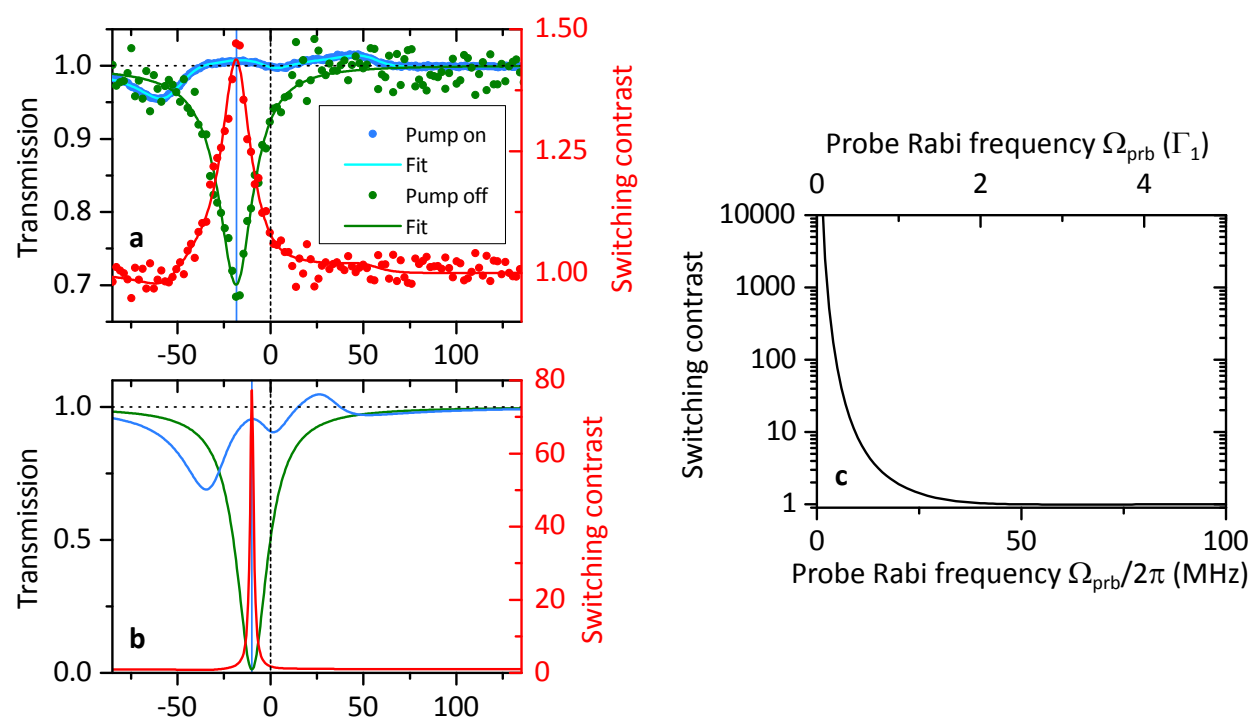

Pump-probe detuning $\delta / 2 \pi(\mathrm{MHz})$

Figure 4.21: a, Probe beam transmission signal without (green) and with (blue) pump light at $\Delta / 2 \pi=18 \mathrm{MHz}$ and $\Omega_{\mathrm{pmp}} / 2 \pi=50 \mathrm{MHz}$. The switching contrast is shown by the red curve, which employs the axis on the right-hand side. The solid lines are fits to the data. $\mathbf{b}$, as in $\mathbf{a}$, but for perfect coupling. The pump beam parameters for the blue curve are $\Omega_{\mathrm{pmp}} / 2 \pi=30 \mathrm{MHz}$ and $\Delta / 2 \pi=10 \mathrm{MHz}$. The vertical solid blue and dotted black lines indicate the frequency of the unperturbed transition and the pump beam, respectively. c, Switching contrast with pump beam parameters as in $\mathbf{b}$, but variable probe Rabi frequency. From [42] with changes.

When the pump beam is turned on $\left(\Omega_{\mathrm{pmp}} / 2 \pi=50 \mathrm{MHz}, \Delta / 2 \pi=\right.$ $18 \mathrm{MHz}$ ), the transmission of the probe beam is greatly increased as shown by the blue curve. In this fashion, the pump beam can switch the transmission of the probe beam. We define a switching contrast as $T_{\text {on }} / T_{\text {off }}$ to quantify this behavior. Here, $T_{\text {on }}$ and $T_{\text {off }}$ denote the transmitted probe power with the pump beam turned on and off, respectively. We reach a maximum switching contrast of 1.5 corresponding to $1.8 \mathrm{~dB}$ at the resonance frequency of the unperturbed molecule, as shown by the red curve in Fig. 4.21 a.

If we assume perfect coupling, combined with the detection through a narrow-band cavity to remove the pump beam and incoherent fluorescence from the molecule, we arrive at the situation shown in part $\mathbf{b}$. With a pump Rabi frequency of only $30 \mathrm{MHz}$ and $\Omega_{\mathrm{prb}} / 2 \pi=5 \mathrm{MHz}$, corresponding to just above 1 pump photon per excited state lifetime, we obtain a switching contrast of 80 or $19 \mathrm{~dB}$. 


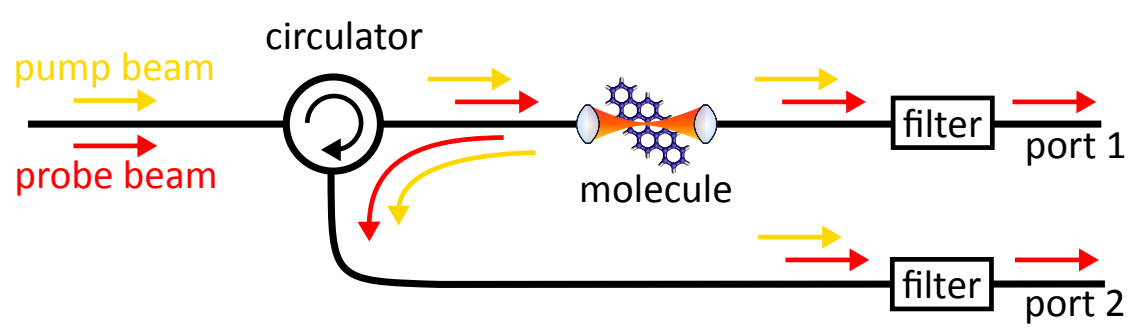

Figure 4.22: Schematic of a single molecule optical router. See text for explanation. filter: narrow-band spectral filter.

An interesting feature of our single molecule switch is the fact that it has two output ports: If the pump beam is turned off, the probe beam is reflected by the molecule [66]. While if the pump is switched on, the probe beam is transmitted. Figure 4.22 depicts how this property could be used to construct a single molecule optical router. Pump and probe light is focused onto a molecule after passing an optical circulator. The circulator directs light that is reflected by the molecule into output port 2, while the transmitted light arrives in output port 1 . Narrow spectral filters remove the pump light in the output modes. Using such a setup enables a weak pump beam to control the direction of another beam [141].

The switching contrast is a function of the probe beam Rabi frequency, as depicted in Fig. $4.21 \mathrm{c}$. This behavior is mainly caused by the reduced extinction signal for large $\Omega_{\text {prb }}$ (compare Fig. 2.7). A switching contrast above 10 is only observed for $\Omega_{\mathrm{prb}} / 2 \pi<10 \mathrm{MHz}=\Gamma_{1} / 2$. This corresponds to a $1 / 8$ photon per lifetime. However, the equivalent absolute power is a function of the excited state lifetime. It can be decreased by placing the molecule inside a micro-cavity [142] or close to a plasmonic nano-structure [81]. In that way, the usable input power can be adjusted to match the specific needs.

\subsection{Time-dependent measurements}

\subsubsection{Setup modifications and measurement technique}

Monitoring an optical signal that changes at a rate of $100 \mathrm{MHz}$ (corresponding to a period of $10 \mathrm{~ns}$ ) is usually not a problem, as state of the art photodiodes can even resolve frequencies of more than $10 \mathrm{GHz}$. The challenge in our case, is the weak power of the transmitted beam. It consists of only one million photons per second, meaning that on average only every 1ooth oscillation cycle contains a photon. Observation of such faint light beams requires the use 

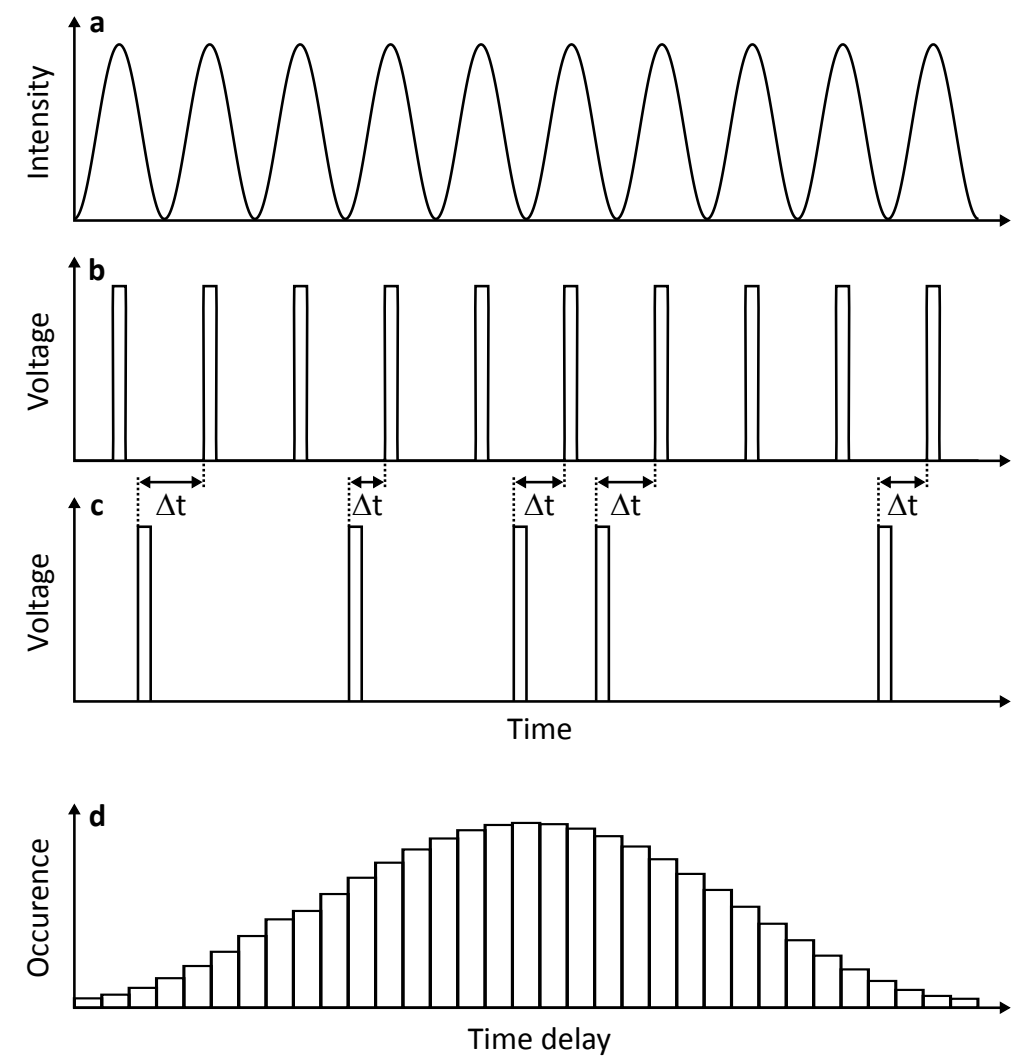

Figure 4.23: Working principle of the time-dependent measurements. a, Reference signal. $\mathbf{b}$, The reference signal of a is converted into a series of pulses. c, Pulses generated by the detection of single photons. $\Delta t$ marks the time delay between a photon pulse and the next reference pulse. Plots a - $\mathbf{c}$ share a common time axis. $\mathbf{d}$, Histogram of time delays $\Delta t$.

of sensitive single photon counting modules. Their time resolution, however, is limited by the so-called dead time, which renders the detector unresponsive to light for roughly $50 \mathrm{~ns}$ after the registration of a photon. Thus, it is not possible to directly resolve variations on a nanosecond scale.

To circumvent this problem, we take advantage of the signal periodicity. By measuring the arrival time of each photon relative to a reference with the same oscillation period and fixed phase relation, we can reconstruct the time evolution of the original signal [96].

The working principle of this method is depicted in Fig. 4.23. A reference signal (a) is converted into a series of pulses (b). The weak signal, whose time dependence should be measured, is detected with a SPCM. The arrival of each photon generates a short electric pulse (c). Instead of just counting the 
number of pulses within a certain integration time, the delay $\Delta t$ between the photon arrival and the next reference pulse is recorded. Using the measured time delays, a histogram can be created that reflects the time dependence of the weak signal (d).

Figure 4.24 a shows how the reference is obtained for the transmission measurement. Before the two beams enter the cryostat, a part of pump (yellow) and probe beams (red) is split off and superposed on a fast and sensitive linear-mode avalanche photodiode (Menlo Systems). In the ideal case, the resulting beating signal has a fixed phase relation to the relative phase of pump and probe fields at the position of the molecule. However, refractive index fluctuations, e.g., due to temperature or air pressure changes within the interferometer (blue shaded area), can cause a variation of the optical path difference for pump and probe. This leads to a modification of the phase relation and hence to a blurring of the time-dependent signal. To reduce the impact of these fluctuations, the size of the interferometer should be minimized. Experimental constraints lead to rather large interferometer arms of around $1 \mathrm{~m}$ and the resulting phase jitter is approximately $15^{\circ}$.

For the time-resolved excited state population measurements, the jitter can be vastly reduced. In that case, it is not necessary to separate pump and probe beams after the cryostat because the signal is the red-shifted fluorescence of the molecule. Hence, pump and probe can share the same optical path, rendering their relative phase insensitive to refractive index fluctuations. To ensure perfect mode overlap, pump and probe beams are coupled to single-mode optical fibers, which are combined at a fiber-coupled beam splitter (see Fig. 4.24 b). One output port is used as reference signal, while the light in the other port is sent into the cryostat. The fluorescence signal is separated from the excitation light with a long-pass filter and detected using a SPCM.

The time difference between the photon arrival and the reference signal is measured with a time-correlated single photon counting (TCSPC) system (Picoquant). To convert the reference beating into a series of pulses, in accordance with the TCSPC system requirements, the beating signal is amplified (Mini-Circuits) and then employed as trigger for a time delay and pulse generator (Stanford Research Systems). Whenever the beating signal crosses a specific threshold with a positive slope, a short rectangular pulse is generated. If this is done for every cycle of the reference oscillation, the maximum measurable time delay is limited to one beating period. To record several cycles of the signal, the pulse generator is configured to ensure a minimum time delay between subsequent reference pulses of $2 \mu \mathrm{s}$. Hence, depending on the exact 

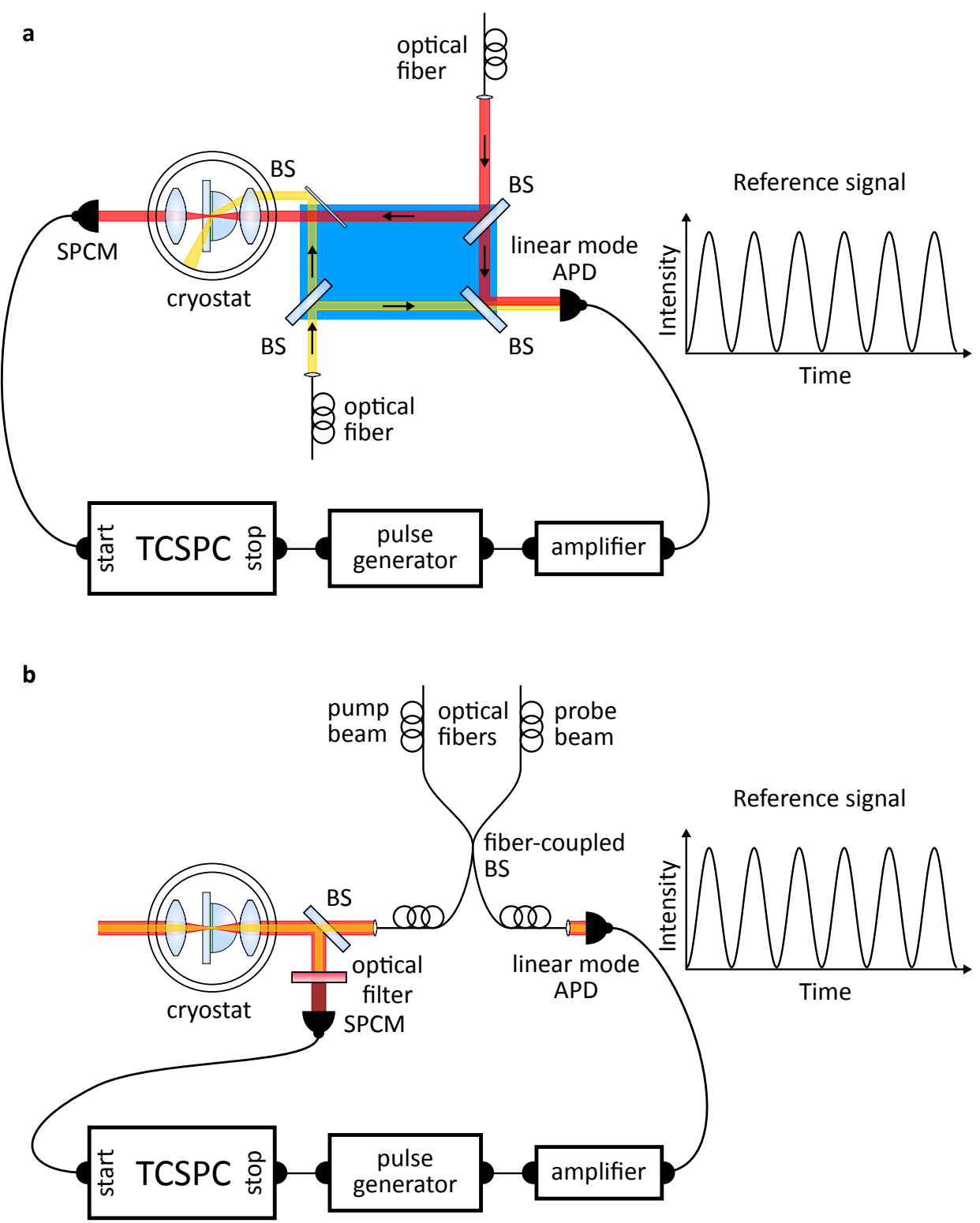

Figure 4.24: Schematic of the time-dependent measurement of the transmission (a) and the fluorescence signal (b). The blue shaded region in a indicates the part that is sensitive to refractive index fluctuations. See text for a detailed explanation. BS: beam splitter, SPCM: single photon counting module, APD: avalanche photodiode, TCSPC: time correlated single photon counting system. 
value of the pump-probe detuning, between 1 and 200 periods of the beating signal are skipped until the next reference pulse is generated.

The pulse generated by the SPCM after the detection of a photon constitutes the start signal for the TCSPC system, while the pulse deduced from the reference beating stops the time measurement. Hence, the TCSPC system measures the delay between the photon arrival and the next reference pulse. If the photon count rate is much smaller than the rate of reference pulses, one can use a histogram of the time delays to describe the time evolution of the weak signal. The time resolution is limited by the timing uncertainty of the SPCM, which is caused by a variation of the time delay between the absorption of a photon and the generation of the output pulse. This so-called transient time spread is around $0.6 \mathrm{~ns}$ [71], more than ten times below the shortest oscillation period we want to observe.

\subsubsection{Experimental results}

We present first the time-dependent probe transmission signal and afterwards the excited state population of a single molecule.

\section{Four-wave mixing}

Figure 4.25 a shows the time-resolved transmission signal for 200 different values of the pump-probe detuning $\delta$. At each detuning, we observe a periodic beating with a frequency given by $\delta$. It can be nicely seen in part $\mathbf{b}$, where a cross section of the time dependent transmission signal for a pump-probe detuning of $50 \mathrm{MHz}$ is depicted. The beating is caused by the interference of residual pump light with the transmitted probe field. As the pump-probe detuning is changed, the periodicity of the beating signal is altered as well. This leads to the pattern visible in $\mathbf{a}$.

To analyze the signal in more detail we perform a Fourier transform of the time-dependent transmission signal as depicted in $\mathbf{c}$ and $\mathbf{d}$. The color code shows the magnitude of the respective frequency component. The dominant feature, labeled as (i), occurs directly at the pump-probe frequency detuning.

More interesting is a second weaker feature at twice the pump-probe detuning, labeled as (ii). The mere existence of this signal indicates a nonlinear interaction of pump and probe beam, as it requires a light field with a new frequency. This new frequency can only originate from the light coherently scattered by the molecule. With Eq. (2.34) we can write the expectation value 

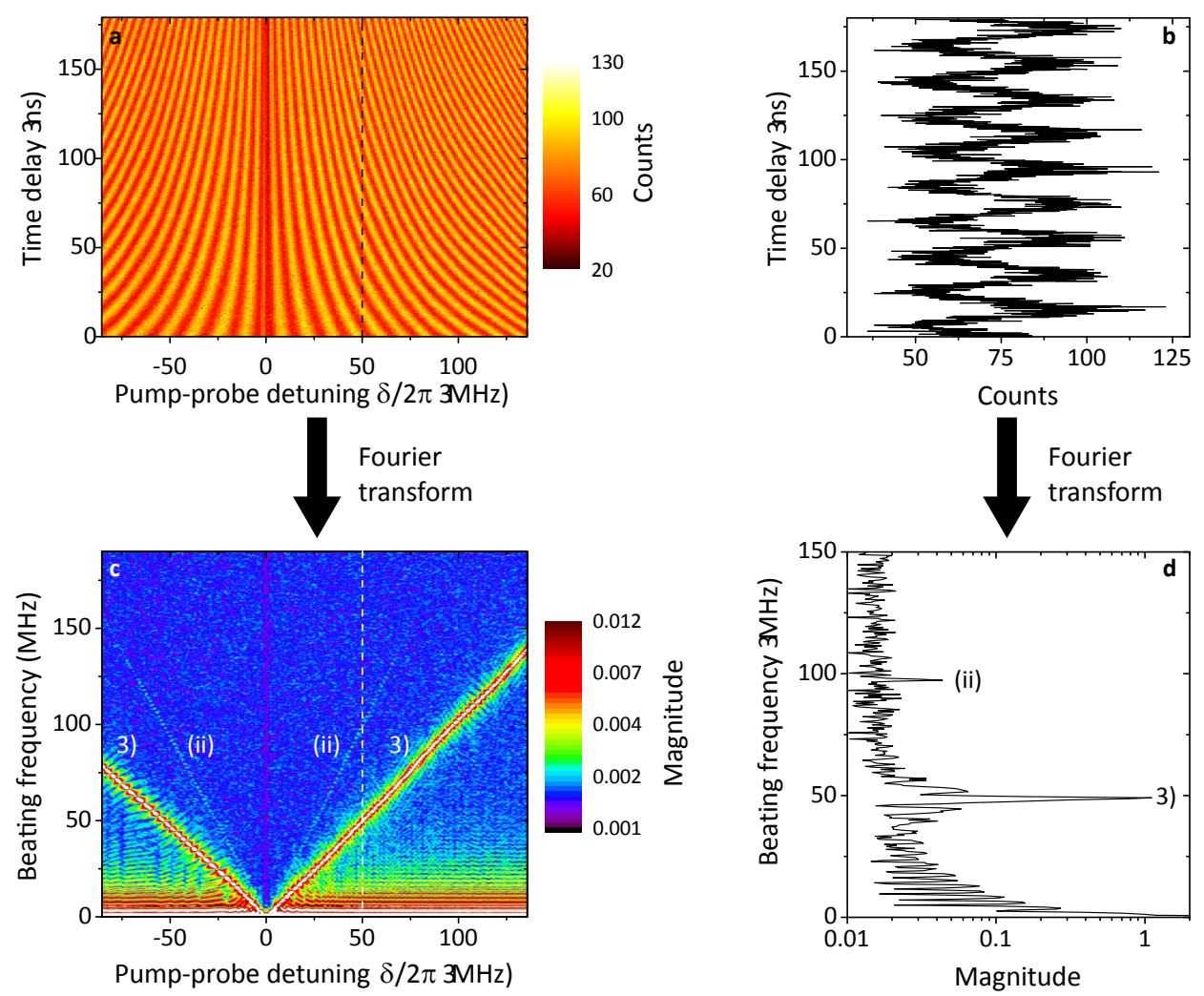

Figure 4.25: Time-dependent transmission signal. $\mathbf{a}$, Transmission signal in time space. $\mathbf{b}$, Transmission signal at a pump-probe detuning of $\delta / 2 \pi=50 \mathrm{MHz}$, as indicated by the dashed vertical line in a. c, Fourier transform of the time-dependent transmission signal shown in a. The color code uses a logarithmic scale. d, Fourier transform of the transmission signal at a pump-probe detuning of $\delta / 2 \pi=50 \mathrm{MHz}$, as indicated by the dashed vertical line in $\mathbf{c}$. The experimental parameters are: $\Omega_{\mathrm{pmp}} / 2 \pi=51 \mathrm{MHz}, \Omega_{\mathrm{prb}} / 2 \pi=8 \mathrm{MHz}$ and $\Delta / 2 \pi=$ $18 \mathrm{MHz}$.

of the scattered field as:

$$
\left\langle\hat{\mathbf{E}}_{\text {scat }}^{-}(\mathbf{r}, t)\right\rangle \propto\left\langle\hat{\sigma}^{\dagger}(t)\right\rangle=\sum_{n=-\infty}^{\infty} \tilde{\rho}_{\mathrm{ge}, n} e^{i\left(\omega_{\mathrm{pmp}}+n \delta\right) t},
$$

where we used Eq. (4.16) in the second step. As can be seen from Eq. (4.38), $\left\langle\hat{\mathbf{E}}_{\text {scat }}^{-}(\mathbf{r}, t)\right\rangle$ is a superposition of light fields of frequency $\omega_{\mathrm{pmp}}+n \delta$, with an amplitude given by $\left|\tilde{\rho}_{\text {ge }, n}\right|$.

Only four of the frequency components could explain the additional beating signal. The components with $\omega_{\mathrm{pmp}}-\delta$ or $\omega_{\mathrm{pmp}}+3 \delta$ could interfere with the probe beam, or the components with $\omega_{\text {pmp }} \pm 2 \delta$ could interfere with the 


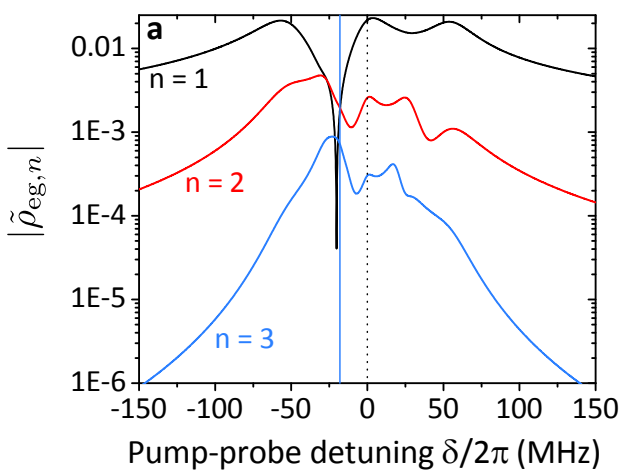

b

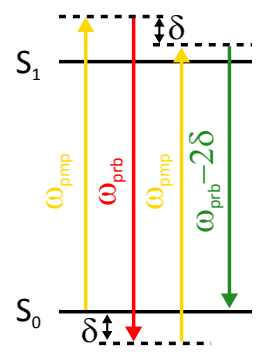

Figure 4.26: a, Amplitude of different frequency components in the field emitted by the molecule. The vertical blue and black lines indicate the frequencies of the unperturbed molecular resonance and of the pump beam, respectively. The parameters are equal to those of Fig. 4.25. b, Level scheme of the four-wave mixing process leading to a new field with frequency $\omega_{\text {prb }}-2 \delta$.

pump beam, to cause a beating signal at $2 \delta$. Figure 4.26 a shows the amplitude of the respective frequency components. As $\left|\tilde{\rho}_{\mathrm{eg}, n}\right|=\left|\tilde{\rho}_{\mathrm{eg},-n}\right|$, it is sufficient to plot three curves. Besides a narrow frequency window around the unperturbed molecular resonance, the absolute value of $\tilde{\rho}_{\text {eg, } 1}$ dominates, which corresponds to the amplitude of the oscillation at $\omega_{\mathrm{pmp}}-\delta=\omega_{\mathrm{prb}}-2 \delta$. A wave at this frequency is generated during the degenerate four-wave mixing process depicted in $\mathbf{b}$ : Two pump photons are absorbed and the probe beam stimulates the emission of a photon at $\omega_{\mathrm{prb}}$, while a photon at $\omega_{\mathrm{prb}}-2 \delta$ is spontaneously emitted [1].

As the pump-probe detuning becomes larger than $70 \mathrm{MHz}$, the beating at $2 \delta$ disappears. Moreover, a reference measurement with pump and probe frequencies far away from the resonance shows no beating at this frequency. Both observations indicate that the four-wave mixing process is indeed enabled by the narrow resonance of a single molecule and not an unknown nonlinear effect, e.g., of the naphthalene matrix or the detector.

\section{Evolution of the excited-state population}

While the time evolution of the transmitted signal reveals the dynamics of the driven molecular dipole, we can use the fluorescence signal to study the time dependence of the excited-state population. The measurement technique is the same as for the transmission signal, but this time the start of a time measurement is triggered by the detection of a fluorescence photon. 


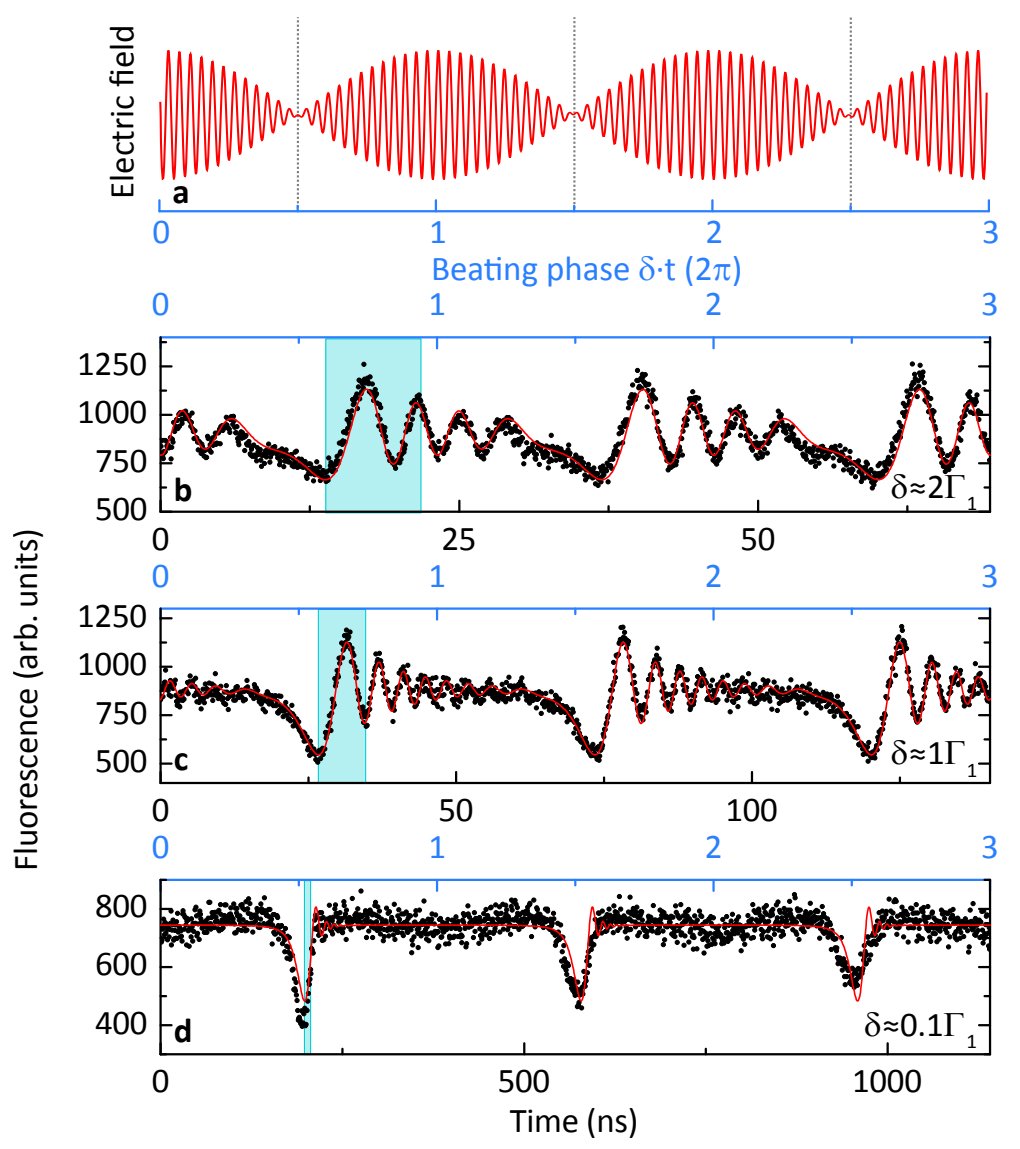

Figure 4.27: Time-dependent excited state population. a, Sketch of the electric field time evolution. $\mathbf{b}$ - d, Fluorescence signal of a single molecule as a function of the beating phase $\delta \cdot t$ (top, blue axis) and time (bottom axis). The pump-probe detuning is varied from $\mathbf{b}$ - $\mathbf{d}$ as indicated by the label. The blue stripes show the lifetime of the excited state. The red solid lines are fits of Eq. (4.2.1) to the data. The data has been corrected for background fluorescence. Figure from [42] with minor changes.

The dynamics of the excited-state population is governed by the interference of pump and probe fields at the position of the molecule as shown in Fig. 4.27 a. When both fields interfere destructively the molecule is only weakly driven, while half a beating period later constructive interference leads to a strong excitation of the molecule. The contrast between weak and strong driving is most pronounced if pump and probe Rabi frequencies are equal. For the measurements shown in Fig. $4.27 \mathrm{~b}-\mathbf{d}$, the experimental parameters are thus $\Omega_{\mathrm{pmp}}=\Omega_{\mathrm{prb}}=2 \pi \cdot 140 \mathrm{MHz}$. For each measurement, the pump beam is on resonance, i.e., $\Delta=0$, while the pump-probe detuning $\delta$ 
is set to a specific value. For better comparison, the fluorescence evolution is plotted as a function of the beating phase $\delta \cdot t$ (blue axis). On this scale, the positions of the constructive and destructive interference are independent of the pump-probe frequency difference. A second axis also shows time for each case (bottom, black axis) to visualize the different time scales involved.

In parts $\mathbf{b}$ and $\mathbf{c}$, the effective Rabi frequency quickly rises to significant values on a time scale much shorter than the excited state lifetime due to the large pump-probe detuning. This causes the excited-state population to undergo Rabi oscillations. As the driving strength changes throughout the beating period, the oscillations are chirped. Their frequency is highest when pump and probe fields are in phase and decreases as destructive interference sets in. The amplitude of the Rabi oscillation is largest when the driving starts after destructive interference and is subsequently damped due to spontaneous emission.

In plot d, the total field increases only slowly and, hence, no Rabi oscillations are observable. As soon as the excitation field saturates the molecule, the excited state population does not change any more and the fluorescence signal stays constant for most of the beating period. Only around complete destructive interference of pump and probe beams, spontaneous decay leads to a drop of the excited state population.

The high intensities of the pump and probe beams lead to the excitation of off-resonant molecules in the focal volume, contributing to a background which is accounted for by a term linear in the intensity of the incident field. By fitting this extended model to the measured data, we can deduce the amount of background fluorescence and correct the measured data accordingly.

Here, we showed the time-dependent response of the excited state population. As the molecule is continuously driven by the pump and probe beams, it is all the time in a quasi-steady-state. The changing population is caused by interference of pump and probe, which lead effectively to excitation of the molecule with an ever changing Rabi frequency.

New compelling features arise, when considering pulsed pump and probe excitation. The usage of pulses allows the observation of the transient features, occurring directly after switching on the driving fields. In contrast to continuous-wave excitation, the molecule always starts in the ground state when the pulses impinge on the molecule. The sudden appearance of the pump beam transfers the molecule into an entangled superposition of different dressed states [143]. 
It was shown that the excited state population depends in that case on the relative phase between pump and probe pulses [143]. A similar dependence should also exist for the transmitted probe power. The phase between pump and probe pulse, thus, adds an additional degree of freedom to control the dynamics of the molecule and the interaction of pump and probe. As these effects disappear within one excited state lifetime, sub-nanosecond pulses are required for the investigation. At the same time, the pulses must not be too short, to still allow spectral selection of a single molecule. Hence, a pulse duration of a few 100 ps would be ideal and also accessible in the required wavelength range by using a pulsed dye laser. 


\section{Outlook}

In this work, we demonstrated few-photon nonlinear optics with a single molecule, enabled by the efficient coupling between excitation light and emitter. We also presented potential applications of this large nonlinearity in terms of a single molecule optical router. In the last part of this thesis, we discuss possible future experimental and theoretical investigations.

Having established control over a single molecule, a natural extension of our work is to study nonlinear optics with "small ensembles". Using widefield illumination, we can acquire position and resonance frequency of many molecules in parallel. Thus, it should be possible to identify molecules close to plasmonic nano-structures or pairs of dipole-coupled molecules. Indeed, there are theoretical investigations of the response of a metal nano-particle quantum dot hybrid system [144, 145] and of dipole-coupled atoms [146] to a bichromatic driving field. These studies can be readily expanded to organic dye molecules and predict novel spectral features in absorption as well as in emission. In the latter case, one remarkable property is the appearance of a new gain feature at moderate pump levels of $\Omega_{\mathrm{pmp}} \approx 2 \Gamma_{1}$ in a spectral region where there is only attenuation for a single emitter. Indeed, it can be shown that the origin of this new peak is the dipole-dipole coupling [146]. In case of the hybrid system, there are two distinct regimes, depending on the distance of emitter and metal nano-particle. If this distance is larger than a critical value of around $15 \mathrm{~nm}$, the pump field experiences plasmonic enhancement corresponding to a driving of the emitter with an increased $\Omega_{\mathrm{pmp}}$. But if the distance is below the critical value, fundamentally different effects occur. In particular, the probe beam experiences pronounced gain in a narrow spectral region around zero pump-probe detuning [144].

Another major task is to bridge the remaining gap to a perfect coupling efficiency. Different possible methods have been pointed out in section 3.3, namely usage of a Gallium phosphide solid immersion lens, a spherical mirror to refocus the light onto the molecule, and an optical antenna structure [125]. While all three approaches demonstrate methods to funnel the light very efficiently to the molecule, the possible coupling is still limited by the Debye-Waller and Franck-Condon factors of the molecule. This puts an upper bound of roughly $50 \%$ onto the coupling efficiency. 
To overcome this constraint, we need to optimize the fluorescence branching ratio. This can be achieved by using a cavity that is in resonance with the ZPL. The presence of the cavity modifies the local density of states, leading to a Purcell enhancement of the decay rate of the resonant transition [147]. A Purcell factor $F_{P}$ of 100 would increase the fraction of the emission into the ZPL from 50\% to more than 99\% [148]. The Purcell factor is determined by the quality factor $Q$ and the mode volume $V$ of the cavity, and the transition wavelength $\lambda$. It is given by [147]:

$$
F_{P}=\frac{3 \lambda^{3}}{4 \pi} \frac{Q}{V}
$$

It is evident from Eq. (5.1) that the Purcell enhancement is optimal for a cavity with small mode volume and large quality factor. However, combining these two properties is a big technological challenge and so far no cavity is operating in the high-Q low-V limit [142]. What remains is the optimization of one of the two values, while keeping the other one in a moderate regime.

A large quality factor leads to very narrow resonance lines which are not ideal for the combination with solid-state emitters for two main reasons: Firstly, the linewidth of the emitter should be smaller than the resonance of the cavity to achieve an optimal Purcell factor. Secondly, the transition frequencies of solid state emitters are spread over the inhomogeneous distribution which is typically much broader than the resonance of a high-Q cavity. Hence, the cavity would have to be tuned differently for each emitter [142].

Better suited for the combination with solid-state emitters is the approach of a low-Q cavity with a very small mode volume. The low quality factor resolves the problems connected with a narrow cavity resonance, while the small mode volume could still lead to a significant Purcell enhancement. Achieving a mode volume close to the fundamental limit of $(\lambda / 2)^{3}$ requires the mirrors to be separated by less than a micro-meter. One possible realization of such a micro-cavities, is the combination of a high numerical aperture, micron-sized curved mirror with a flat distributed Bragg reflector as second cavity mirror [142]. By placing a thin dye-doped crystal in such a cavity, one could potentially achieve a near optimal coupling efficiency.

Such a significantly improved coupling efficiency would open the door to light-matter interaction at its most fundamental level, where single photons interact with a single emitter. A theoretical study by Kochan and Carmichael [120] predicted that the transmission signal depends in this regime on the 

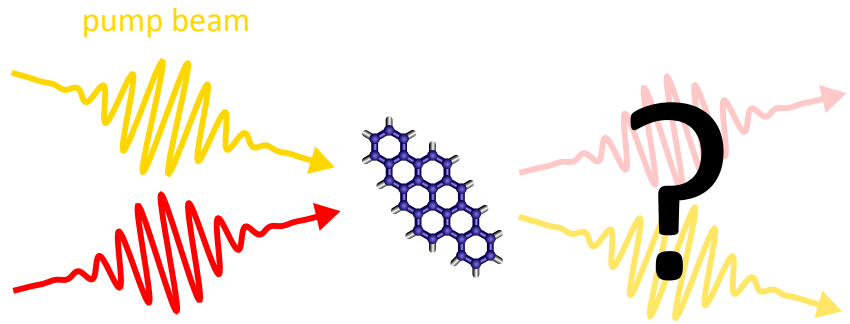

probe beam

Figure 5.1: Schematic of the fundamental interaction of two photons with a single molecule.

photon statistics of the incident light. Yet, the observation requires a coupling efficiency of greater than $80 \%$. Otherwise, "the [photon] statisticsdependent features are swamped by the flux of photons which [...] pass outside the absorption cross section" [120].

The calculations in Ref. [120] are limited to a single beam with narrow linewidth. An intriguing question is how the nonlinear interaction of two beams would change if both beams contained only a single photon, as depicted in Fig. 5.1. The theoretical treatment presented in this work uses coherent states for the pump and probe beams. Even if we ignored the predictions by Kochan and Carmichael and assumed that incident single photons would behave like a weak coherent beam of same power, our calculations are still only valid for an excitation field with a narrow linewidth.

This is very different from a photon that is emitted by a single molecule, whose linewidth and temporal shape would be determined by the optical transition. A study by Stobińska and coworkers [149] has shown that the interaction of a single photon and a single emitter largely depends on the spectral and temporal shape of the photon. Given the appropriate shape, a single photon can, e.g., perfectly excite a single emitter.

If we imagine a single pump photon with this shape, the molecule would be transfered into the excited state and could amplify the probe beam via stimulated emission. In this case, the probe beam would double in power a much stronger amplification than it is possible via the three photon process presented in chapter 4 . But what happens if the probe photon arrives, while the pump photon is still exciting the molecule? How does the shape of the probe photon influence this process. What happens if pump and probe photons have different temporal shapes? For now, answering these questions remains an open task for future works dealing with nonlinear optics on the ultimate quantum level. 



\section{Bibliography}

1. Boyd, R. W. Nonlinear Optics (Elsevier Inc., 2008).

2. Lewis, G. N., Lipkin, D. \& Magel, T. T. Reversible Photochemical Processes in Rigid Media. A Study of the Phosphorescent State. Journal of the American Chemical Society 63, 3005-3018 (Nov. 1941).

3. Franken, P. A. \& Ward, J. F. Optical Harmonics and Nonlinear Phenomena. Reviews of Modern Physics 35, 23-39 (Jan. 1963).

4. Burnham, D. C. \& Weinberg, D. L. Observation of Simultaneity in Parametric Production of Optical Photon Pairs. Physical Review Letters 25, 84-87 (July 1970).

5. Wieczorek, W. et al. Experimental Entanglement of a Six-Photon Symmetric Dicke State. Physical Review Letters 103, 020504 (July 2009).

6. Kelley, P. L. Self-Focusing of Optical Beams. Phys. Rev. Lett. 15, 10051008 (26 Dec. 1965).

7. Brewer, R. G. Frequency Shifts in Self-Focused Light. Phys. Rev. Lett. 19, 8-10 (1 July 1967).

8. Shimizu, F. Frequency Broadening in Liquids by a Short Light Pulse. Phys. Rev. Lett. 19, 1097-1100 (19 Nov. 1967).

9. Cheung, A. C., Rank, D. M., Chiao, R. Y. \& Townes, C. H. Phase Modulation of $Q$-Switched Laser Beams in Small-Scale Filaments. Phys. Rev. Lett. 2o, 786-789 (15 Apr. 1968).

10. Kiess, T. E., Shih, Y. H., Sergienko, A. V. \& Alley, C. O. EinsteinPodolsky-Rosen-Bohm experiment using pairs of light quanta produced by type-II parametric down-conversion. Phys. Rev. Lett. 71, 3893-3897 (24 Dec. 1993).

11. Kwiat, P. G. et al. New High-Intensity Source of Polarization-Entangled Photon Pairs. Physical Review Letters 75, 4337-4341 (1995).

12. Lvovsky, A. I. et al. Quantum State Reconstruction of the SinglePhoton Fock State. Phys. Rev. Lett. 87, 050402 (5 July 2001). 
13. Rangarajan, R., Vicent, L. E., U'Ren, A. B. \& Kwiat, P. G. Engineering an ideal indistinguishable photon-pair source for optical quantum information processing. Journal of Modern Optics 58, 318-327 (Feb. 2011).

14. Machinet, G. et al. High-brightness fiber laser-pumped 68 fs-2.3 W Kerr-lens mode-locked $\mathrm{Yb}: \mathrm{CaF}_{2}$ oscillator. Opt. Lett. 38, 4008-4010 (Oct. 2013).

15. Shen, Y. R. The Principles of Nonlinear Optics (John Wiley \& Sons, Inc., Hoboken, 2003).

16. Mukamel, S. Principles of Nonlinear Optical Spectroscopy (Oxford University Press, 1999).

17. Brasselet, S. et al. In Situ Diagnostics of the Crystalline Nature of Single Organic Nanocrystals by Nonlinear Microscopy. Physical Review Letters 92, 207401 (2004).

18. Lippitz, M., van Dijk, M. A. \& Orrit, M. Third-Harmonic Generation from Single Gold Nanoparticles. Nano Letters 5, 799-802 (Apr. 2005).

19. Horneber, A. et al. Nonlinear optical imaging of single plasmonic nanoparticles with $30 \mathrm{~nm}$ resolution. en. Physical Chemistry Chemical Physics 17, 21288-21293 (Aug. 2015).

20. Mertz, J., Xu, C. \& Webb, W. W. Single-molecule detection by twophoton-excited fluorescence. en. Optics Letters 2o, 2532 (Dec. 1995).

21. Plakhotnik, T., Walser, D., Pirotta, M., Renn, A. \& Wild, U. P. Nonlinear Spectroscopy on a Single Quantum System: Two-Photon Absorption of a Single Molecule. en. Science 271, 1703-1705 (Mar. 1996).

22. Gerhardt, I. et al. Coherent state preparation and observation of Rabi oscillations in a single molecule. Physical Review A 79, 011402 (Jan. 2009).

23. Gerhardt, I., Wrigge, G., Hwang, J., Zumofen, G. \& Sandoghdar, V. Coherent nonlinear single-molecule microscopy. Physical Review A 82, 063823 (Dec. 2010).

24. Tamarat, P. et al. Pump-Probe Experiments with a Single Molecule: acStark Effect and Nonlinear Optical Response. Physical Review Letters 75, 1514-1517 (Aug. 1995).

25. Lounis, B., Jelezko, F. \& Orrit, M. Single Molecules Driven by Strong Resonant Fields: Hyper-Raman and Subharmonic Resonances. Physical Review Letters 78, 3673-3676 (May 1997). 
26. Chang, D. E., Vuletić, V. \& Lukin, M. D. Quantum nonlinear optics photon by photon. en. Nature Photonics 8, 685-694 (Sept. 2014).

27. Miller, D. A. B. Are optical transistors the logical next step? en. Nature Photonics 4, 3-5 (Jan. 2010).

28. Kimble, H. J. The quantum internet. en. Nature 453, 1023-1030 (June 2008).

29. Loss, D. \& DiVincenzo, D. P. Quantum computation with quantum dots. Physical Review A 57, 120-126 (Jan. 1998).

30. Gorniaczyk, H., Tresp, C., Schmidt, J., Fedder, H. \& Hofferberth, S. Single-Photon Transistor Mediated by Interstate Rydberg Interactions. Physical Review Letters 113, 053601 (July 2014).

31. Tiarks, D., Baur, S., Schneider, K., Dürr, S. \& Rempe, G. Single-Photon Transistor Using a Förster Resonance. Physical Review Letters 113, 053602 (July 2014).

32. Chang, D. E., Sørensen, A. S., Demler, E. A. \& Lukin, M. D. A single-photon transistor using nanoscale surface plasmons. en. Nature Physics 3, 807-812 (Nov. 2007).

33. Reiserer, A., Ritter, S. \& Rempe, G. Nondestructive Detection of an Optical Photon. en. Science 342, 1349-1351 (Dec. 2013).

34. Turchette, Q. A., Hood, C. J., Lange, W., Mabuchi, H. \& Kimble, H. J. Measurement of Conditional Phase Shifts for Quantum Logic. Physical Review Letters 75, 4710-4713 (Dec. 1995).

35. Kalb, N., Reiserer, A., Ritter, S. \& Rempe, G. Heralded Storage of a Photonic Quantum Bit in a Single Atom. Physical Review Letters 114, 220501 (June 2015).

36. Vahala, K. J. Optical microcavities. en. Nature 424, 839-846 (Aug. 2003).

37. Armani, D. K., Kippenberg, T. J., Spillane, S. M. \& Vahala, K. J. Ultrahigh-Q toroid microcavity on a chip. en. Nature 421, 925-928 (Feb. 2003).

38. Volz, J., Scheucher, M., Junge, C. \& Rauschenbeutel, A. Nonlinear $\pi$ phase shift for single fibre-guided photons interacting with a single resonator-enhanced atom. en. Nature Photonics 8, 965-970 (Dec. 2014).

39. Michler, P. et al. A Quantum Dot Single-Photon Turnstile Device. en. Science 290, 2282-2285 (Dec. 2000). 
40. Fushman, I. et al. Controlled Phase Shifts with a Single Quantum Dot. en. Science 320, 769-772 (May 2008).

41. Volz, T. et al. Ultrafast all-optical switching by single photons. en. Nature Photonics 6, 605-609 (Sept. 2012).

42. Maser, A., Gmeiner, B., Utikal, T., Götzinger, S. \& Sandoghdar, V. Fewphoton coherent nonlinear optics with a single molecule. Nature Photonics 10, 450-453 (July 2016).

43. Mollow, B. R. Stimulated Emission and Absorption near Resonance for Driven Systems. Physical Review A 5, 2217-2222 (May 1972).

44. Moerner, W. E. \& Kador, L. Optical detection and spectroscopy of single molecules in a solid. Physical Review Letters 62, 2535-2538 (May 1989).

45. Orrit, M. \& Bernard, J. Single pentacene molecules detected by fluorescence excitation in a p-terphenyl crystal. Physical Review Letters $\mathbf{6 5}_{5}$, 2716-2719 (Nov. 1990).

46. Betzig, E. \& Chichester, R. J. Single Molecules Observed by Near-Field Scanning Optical Microscopy. en. Science 262, 1422-1425 (Nov. 1993).

47. Nie, S., Chiu, D. T. \& Zare, R. N. Probing individual molecules with confocal fluorescence microscopy. en. Science 266, 1018-1021 (Nov. 1994).

48. Ishijima, A. \& Yanagida, T. Single molecule nanobioscience. Trends in Biochemical Sciences 26, 438-444 (July 2001).

49. Weisenburger, S. \& Sandoghdar, V. Light microscopy: an ongoing contemporary revolution. Contemporary Physics 56, 123-143 (Apr. 2015).

50. The Nobel Prize in Chemistry 2014 - Press Release <http : / / www - nobelprize . org / nobel_prizes / chemistry / laureates/2014/press.html> (2015).

51. Kulzer, F., Xia, T. \& Orrit, M. Single Molecules as Optical Nanoprobes for Soft and Complex Matter. en. Angewandte Chemie International Edition 49, 854-866 (Jan. 2010).

52. Werley, C. A. \& Moerner, W. E. Single-Molecule Nanoprobes Explore Defects in Spin-Grown Crystals. The Journal of Physical Chemistry B 110, 18939-18944 (Sept. 2006).

53. Bloeß, A., Durand, Y., Matsushita, M., Schmidt, J. \& Groenen, E. J. J. A single-molecule study of the relation between the resonance frequency and the orientation of a guest molecule in a Shpol'skii system. Chemical Physics Letters 344, 55-6o (Aug. 2001). 
54. Basché, T., Moerner, W. E., Orrit, M. \& Talon, H. Photon antibunching in the fluorescence of a single dye molecule trapped in a solid. Physical Review Letters 69, 1516-1519 (Sept. 1992).

55. Basché, T. \& Moerner, W. E. Optical modification of a single impurity molecule in a solid. en. Nature 355, 335-337 (Jan. 1992).

56. Moerner, W. E. et al. Optical Probing of Single Molecules of Terrylene in a Shpol'kii Matrix: A Two-State Single-Molecule Switch. The Journal of Physical Chemistry 98, 7382-7389 (July 1994).

57. Basché, T., Kummer, S. \& Bräuchle, C. Direct spectroscopic observation of quantum jumps of a single molecule. en. Nature 373, 132-134 (Jan. 1995).

58. Hettich, C. et al. Nanometer Resolution and Coherent Optical Dipole Coupling of Two Individual Molecules. en. Science 298, 385-389 (Oct. 2002).

59. Mollow, B. R. Power Spectrum of Light Scattered by Two-Level Systems. Physical Review 188, 1969-1975 (Dec. 1969).

6o. Wrigge, G., Gerhardt, I., Hwang, J., Zumofen, G. \& Sandoghdar, V. Efficient coupling of photons to a single molecule and the observation of its resonance fluorescence. en. Nature Physics 4, 6o-66 (Jan. 2008).

61. Hwang, J. et al. A single-molecule optical transistor. en. Nature 46o, 76-8o (July 2009).

62. Pototschnig, M. et al. Controlling the Phase of a Light Beam with a Single Molecule. Physical Review Letters 107, 063001 (Aug. 2011).

63. Lettow, R. et al. Quantum Interference of Tunably Indistinguishable Photons from Remote Organic Molecules. Physical Review Letters 104, 123605 (Mar. 2010).

64. Rezus, Y. L. A. et al. Single-Photon Spectroscopy of a Single Molecule. Physical Review Letters 108, 093601 (Feb. 2012).

65. Siyushev, P., Stein, G., Wrachtrup, J. \& Gerhardt, I. Molecular photons interfaced with alkali atoms. en. Nature 509, 66-70 (May 2014).

66. Zumofen, G., Mojarad, N. M., Sandoghdar, V. \& Agio, M. Perfect Reflection of Light by an Oscillating Dipole. Physical Review Letters 101, 180404 (Oct. 2008).

67. Jackson, J. D. Classical electrodynamics (Wiley, New York, 2002).

68. Plakhotnik, T., Donley, E. A. \& Wild, U. P. Single-Molecule Spectroscopy. Annual Review of Physical Chemistry 48, 181-212 (1997). 
69. Moerner, W. E. \& Orrit, M. Illuminating Single Molecules in Condensed Matter. en. Science 283, 1670-1676 (Mar. 1999).

70. Loudon, R. The Quantum Theory of Light (Oxford Scientific Publications, 2010).

71. Wrigge, G. Coherent and Incoherent Light Scattering in the Resonance Fluorescence of a Single Molecule PhD thesis (ETH Zürich, 2008).

72. Friedrich, J. \& Haarer, D. Photochemical Hole Burning: A Spectroscopic Study of Relaxation Processes in Polymers and Glasses. en. Angewandte Chemie International Edition in English 23, 113-140 (Feb. 1984).

73. Basché, T., Moerner, W. E., Orrit, M. \& Wild, U. P. Single-Molecule Optical Detection, Imaging and Spectroscpy (VCH Verlagsgesellschaft $\mathrm{mbH}$, Weinheim, 1997).

74. Weissbluth, M. Atoms and Molecules (Academic Press, New York, 1978).

75. Tian, Y., Navarro, P. \& Orrit, M. Single Molecule as a Local Acoustic Detector for Mechanical Oscillators. Physical Review Letters 113, 135505 (Sept. 2014).

76. Faez, S., van der Molen, S. J. \& Orrit, M. Optical tracing of multiple charges in single-electron devices. Physical Review B 90, 205405 (Nov. 2014).

77. Veshchunov, I. S. et al. Direct Evidence of Flexomagnetoelectric Effect Revealed by Single-Molecule Spectroscopy. Physical Review Letters 115, 027601 (July 2015).

78. Eichhammer, E. Detection of a single praseodymium ion in an yttriumorthosilicate crystal (Master thesis, Friedrich-Alexander Universität Erlangen-Nürnberg, 2013).

79. Ambrose, W. P., Basché, T. \& Moerner, W. E. Detection and spectroscopy of single pentacene molecules in a p-terphenyl crystal by means of fluorescence excitation. The Journal of Chemical Physics 95, 7150-7163 (Nov. 1991).

8o. Akimov, A. V. et al. Generation of single optical plasmons in metallic nanowires coupled to quantum dots. en. Nature 450, 402-406 (Nov. 2007). 
81. Lee, K.-G. et al. Spontaneous emission enhancement of a single molecule by a double-sphere nanoantenna across an interface. Optics Express 20, 23331-23338 (Oct. 2012).

82. Chen, X.-W., Sandoghdar, V. \& Agio, M. Coherent Interaction of Light with a Metallic Structure Coupled to a Single Quantum Emitter: From Superabsorption to Cloaking. Physical Review Letters 110, 153605 (Apr. 2013).

83. Hartsfield, T. et al. Single quantum dot controls a plasmonic cavity's scattering and anisotropy. en. Proceedings of the National Academy of Sciences 112, 12288-12292 (Oct. 2015).

84. Jelezko, F., Lounis, B. \& Orrit, M. Pumpprobe spectroscopy and photophysical properties of single di-benzanthanthrene molecules in a naphthalene crystal. The Journal of Chemical Physics 107, 1692-1702 (Aug. 1997).

85. Bloess, A. et al. Microscopic Structure in a Shpol'skii System: A SingleMolecule Study of Dibenzanthanthrene in n-Tetradecane. The Journal of Physical Chemistry A 105, 3016-3021 (Apr. 2001).

86. Boiron, A.-M., Lounis, B. \& Orrit, M. Single molecules of dibenzanthanthrene in n-hexadecane. The Journal of Chemical Physics 105, 3969-3974 (Sept. 1996).

87. Gerhardt, I. et al. Strong Extinction of a Laser Beam by a Single Molecule. Physical Review Letters 98, 033601 (Jan. 2007).

88. Walser, A., Renn, A., Götzinger, S. \& Sandoghdar, V. Lifetime-limited zero-phonon spectra of single molecules in methyl methacrylate. Chemical Physics Letters 472, 44-47 (Apr. 2009).

89. Allen, L. \& Eberly, J. H. Optical Resonance and Two-Level Atoms (Dover Publications, Inc., New York, 1987).

9o. Cohen-Tannoudji, C., Jacques, D.-R. \& Grynberg, G. Atom-Photon Interactions (Wiley-VCH, Weinheim, 2004).

91. Bloch, F. \& Siegert, A. Magnetic Resonance for Nonrotating Fields. Physical Review 57, 522-527 (Mar. 1940).

92. Agio, M. \& Alù, A. Optical Antennas (Cambridge University Press, 2013).

93. Berman, P. R. Cavity Quantum Electrodynamics (Academic Press, 1994). 
94. Press, D. et al. Photon Antibunching from a Single Quantum-DotMicrocavity System in the Strong Coupling Regime. Physical Review Letters 98, 117402 (Mar. 2007).

95. Scully, M. O. \& Zubairy, M. S. Quantum Optics (Cambridge University Press, 1997).

96. Pototschnig, M. Controlling Amplitude and Phase of a Laser Beam by a Single Molecule PhD thesis (ETH Zürich, 2011).

97. Van Enk, S. J. Atoms, dipole waves, and strongly focused light beams. Physical Review A 69, 043813 (Apr. 2004).

98. Wrigge, G., Hwang, J., Gehardt, I., Zumofen, G. \& Sandoghdar, V. Exploring the limits of single emitter detection in fluorescence and extinction. en. Optics Express 16, 17358 (Oct. 2008).

99. Michaelis, J. Mikroskopie mit einem einzelnen Molekül als Lichtquelle $\mathrm{PhD}$ thesis (Universität Konstanz, 2000).

10o. Hettich, C. Coherent Optical Dipole Coupling of Two Individual Molecules at Nanometre Separation PhD thesis (Universität Konstanz, 2002).

101. Gerhardt, I. Scattering \& Absorption of Light by a Single Molecule under a Subwavelength Aperture $\mathrm{PhD}$ thesis (ETH Zürich, 2006).

102. Mansfield, S. M. \& Kino, G. S. Solid immersion microscope. Applied Physics Letters 57, 2615-2616 (Dec. 1990).

103. Wood, D. L. \& Nassau, K. Refractive index of cubic zirconia stabilized with yttria. en. Applied Optics 21, 2978 (Aug. 1982).

104. Mawatari, K. et al. Extended-Nanofluidic Systems for Chemistry and Biotechnology (Imperial College Press, London, 2012).

105. Faez, S., Türschmann, P., Haakh, H. R., Götzinger, S. \& Sandoghdar, V. Coherent Interaction of Light and Single Molecules in a Dielectric Nanoguide. Physical Review Letters 113, 213601 (Nov. 2014).

106. Plakhotnik, T. \& Palm, V. Interferometric Signatures of Single Molecules. Physical Review Letters 87, 183602 (Oct. 2001).

107. Richards, B. \& Wolf, E. Electromagnetic Diffraction in Optical Systems. II. Structure of the Image Field in an Aplanatic System. en. Proceedings of the Royal Society of London A: Mathematical, Physical and Engineering Sciences 253, 358-379 (Dec. 1959). 
108. Bauer, T., Orlov, S., Peschel, U., Banzer, P. \& Leuchs, G. Nanointerferometric amplitude and phase reconstruction of tightly focused vector beams. en. Nature Photonics 8, 23-27 (Jan. 2014).

109. Stallinga, S. \& Rieger, B. Position and orientation estimation of fixed dipole emitters using an effective Hermite point spread function model. en. Optics Express 2o, 5896 (Mar. 2012).

110. Prummer, M., Sick, B., Hecht, B. \& Wild, U. P. Three-dimensional optical polarization tomography of single molecules. The Journal of Chemical Physics 118, 9824-9829 (June 2003).

111. Zirzlmeier, J. et al. Singlet fission in pentacene dimers. en. Proceedings of the National Academy of Sciences 112, 5325-5330 (Apr. 2015).

112. Brewer, R. G. \& Shoemaker, R. L. Optical Free Induction Decay. Physical Review A 6, 2001-2007 (Dec. 1972).

113. Brunel, C., Tamarat, P., Lounis, B., Woehl, J. C. \& Orrit, M. Stark Effect on Single Molecules of Dibenzanthanthrene in a Naphthalene Crystal and in a n-Hexadecane Shpol'skii Matrix. The Journal of Physical Chemistry A 103, 2429-2434 (Apr. 1999).

114. Birnbaum, K. M. et al. Photon blockade in an optical cavity with one trapped atom. en. Nature 436, 87-90 (July 2005).

115. Darquié, B. et al. Controlled Single-Photon Emission from a Single Trapped Two-Level Atom. en. Science 309, 454-456 (July 2005).

116. Tey, M. K. et al. Strong interaction between light and a single trapped atom without the need for a cavity. en. Nature Physics 4, 924-927 (Dec. 2008).

117. Leuchs, G. \& Sondermann, M. Light-matter interaction in free space. Journal of Modern Optics 6o, 36-42 (Jan. 2013).

118. Sheppard, C. J. R. \& Larkin, K. G. Optimal Concentration of Electromagnetic Radiation. Journal of Modern Optics 41, 1495-1505 (July 1994).

119. Bassett, I. M. Limit to Concentration by Focusing. Optica Acta: International Journal of Optics 33, 279-286 (1986).

120. Kochan, P. \& Carmichael, H. J. Photon-statistics dependence of singleatom absorption. Physical Review A 50, 1700-1709 (Aug. 1994).

121. Fischer, M. et al. Efficient saturation of an ion in free space. en. Applied Physics B 117, 797-801 (Mar. 2014). 
122. Munsch, M. et al. Dielectric GaAs Antenna Ensuring an Efficient Broadband Coupling between an InAs Quantum Dot and a Gaussian Optical Beam. Physical Review Letters 110, 177402 (Apr. 2013).

123. Arcari, M. et al. Near-Unity Coupling Efficiency of a Quantum Emitter to a Photonic Crystal Waveguide. Physical Review Letters 113, 093603 (Aug. 2014).

124. Chen, X.-W., Götzinger, S. \& Sandoghdar, V. 99\% efficiency in collecting photons from a single emitter. en. Optics Letters 36, 3545 (Sept. 2011).

125. Chu, X.-L. et al. Experimental realization of an optical antenna designed for collecting $99 \%$ of photons from a quantum emitter. en. $\mathrm{Op}$ tica 1, 203 (Oct. 2014).

126. Courtois, J.-Y., Courty, J.-M. \& Mertz, J. C. Internal dynamics of multilevel atoms near a vacuum-dielectric interface. Physical Review A 53, 1862-1878 (Mar. 1996).

127. Born, M. \& Wolf, E. Principles of optics (Cambridge University Press, 1999).

128. Aspnes, D. E. \& Studna, A. A. Dielectric functions and optical parameters of $\mathrm{Si}, \mathrm{Ge}, \mathrm{GaP}, \mathrm{GaAs}, \mathrm{GaSb}, \mathrm{InP}, \mathrm{InAs}$, and InSb from 1.5 to $6.0 \mathrm{eV}$. Physical Review B 27, 985-1009 (Jan. 1983).

129. Golla, A. et al. Generation of a wave packet tailored to efficient free space excitation of a single atom. en. The European Physical Journal D 66, 190 (July 2012).

130. Wu, F. Y., Ezekiel, S., Ducloy, M. \& Mollow, B. R. Observation of Amplification in a Strongly Driven Two-Level Atomic System at Optical Frequencies. Physical Review Letters 38, 1077-1080 (May 1977).

131. Gruneisen, M. T., MacDonald, K. R., Gaeta, A. L., Boyd, R. W. \& Harter, D. J. Energy transfer between laser beams propagating through an atomic vapor. Physical Review A 40, 3464-3467 (Sept. 1989).

132. Papademetriou, S., Chakmakjian, S. \& Stroud, C. R. Optical subharmonic Rabi resonances. Journal of the Optical Society of America B 9, 1182-1188 (July 1992).

133. Xu, X. et al. Coherent Optical Spectroscopy of a Strongly Driven Quantum Dot. en. Science 317, 929-932 (Aug. 2007). 
134. Xu, X. et al. Single Charged Quantum Dot in a Strong Optical Field: Absorption, Gain, and the ac-Stark Effect. Physical Review Letters 101, 227401 (Nov. 2008).

135. Lezama, A., Zhu, Y., Kanskar, M. \& Mossberg, T. W. Radiative emission of driven two-level atoms into the modes of an enclosing optical cavity: The transition from fluorescence to lasing. Physical Review A 41, 15761581 (Feb. 1990).

136. Boyd, R. W., Raymer, M. G., Narum, P. \& Harter, D. J. Four-wave parametric interactions in a strongly driven two-level system. Physical Review A 24, 411-423 (July 1981).

137. Haroche, S. \& Hartmann, F. Theory of Saturated-Absorption Line Shapes. Physical Review A 6, 1280-1300 (Oct. 1972).

138. Grynberg, G. \& Cohen-Tannoudji, C. Central resonance of the Mollow absorption spectrum: physical origin of gain without population inversion. Optics Communications 96, 150-163 (Feb. 1993).

139. Gruneisen, M. T., MacDonald, K. R. \& Boyd, R. W. Induced gain and modified absorption of a weak probe beam in a strongly driven sodium vapor. Journal of the Optical Society of America B 5, 123-129 (Jan. 1988).

140. Khitrova, G., Valley, J. F. \& Gibbs, H. M. Gain-feedback approach to optical instabilities in sodium vapor. Physical Review Letters 6o, 11261129 (Mar. 1988).

141. Shomroni, I. et al. All-optical routing of single photons by a one-atom switch controlled by a single photon. en. Science 345, 903-906 (Aug. 2014).

142. Kelkar, H. et al. Sensing Nanoparticles with a Cantilever-Based Scannable Optical Cavity of Low Finesse and Sub- $\lambda^{3}$ Volume. Physical Review Applied 4, 054010 (Nov. 2015).

143. Wu, Q., Gauthier, D. J. \& Mossberg, T. W. Phase-sensitive dynamics of bichromatically driven two-level atoms. Physical Review A 49, R1519R1522 (Mar. 1994).

144. Sadeghi, S. M. Gain without inversion in hybrid quantum dot-metallic nanoparticle systems. en. Nanotechnology 21, 455401 (Nov. 2010).

145. Sadeghi, S. M. Ultrafast plasmonic field oscillations and optics of molecular resonances caused by coherent exciton-plasmon coupling. English. Physical Review A 88, 013831 (July 2013). 
146. Agarwal, G. S., Narducci, L. M. \& Apostolidis, E. Effects of dispersion forces in optical resonance phenomena. Optics Communications 36 , 285-290 (Feb. 1981).

147. Purcell, E. M. Spontaneous Emission Probabilities at Radio Frequencies. Physical Review 69, 681 (June 1946).

148. Toninelli, C. et al. A scanning microcavity for in situ control of singlemolecule emission. Applied Physics Letters 97, 021107 (July 2010).

149. Stobińska, M., Alber, G. \& Leuchs, G. Perfect excitation of a matter qubit by a single photon in free space. en. EPL (Europhysics Letters) 86, 14007 (2009). 


\section{Acknowledgments}

Viele Menschen haben direkt oder indirekt zum Erfolg dieser Arbeit beigetragen. Daher möchte ich diese Gelegenheit nutzen um meine Dankbarkeit auszudrücken:

- Meiner Frau NAdja und meinen Kindern Tristan und Sophia für ihre Unterstützung und ihr Verständnis, wenn es mal wieder später wurde als geplant, weil "nur noch schnell" eine neue Probe eingebaut werden musste. Nichts konnte mich einen Tag voller Rückschläge schneller vergessen machen als zu meiner Familie nach Hause zu kommen und vom Lachen meiner Kinder begrüßt zu werden.

- VAhid dafür, dass er mir die Chance gegeben hat an diesem anspruchsvollen Experiment zu Arbeiten, für seine Geduld während der langen, ergebnislosen Durststrecken und seine ansteckende Begeisterung für Physik.

- Meinem Laborpartner BenNy für die gute Zusammenarbeit und die kontinuierliche Versorgung mit Schokolade und anderen Süßigkeiten über viele Jahre. Ohne ihn wären diese Experimente nicht möglich gewesen.

- Тов für seine Freundschaft während all der Jahre, seine Unterstützung innerhalb und außerhalb des Labors und nicht zuletzt das Korrekturlesen dieser Arbeit.

- Stephan dafür, dass er immer ein offenes Ohr für meine Sorgen hatte und für unzählige Stunden, in denen wir scheinbar triviale physikalische Fragen diskutierten, um dann festzustellen, dass die Wahrheit doch nicht so einfach ist.

- Harald, Diego and IonNis for taking the time to discuss numerous theoretical problems in great detail.

- Sigi und Jens für zahllose, unterhaltsame Abende, an denen wir auf virtuellen Schlachtfeldern große Siege und bittere Niederlagen erlebt haben. 
- MAKs für seinen virtuosen Umgang mit Fräse und Drehbank. Der auf Hochglanz polierte Probenhalter wird sicherlich noch lange neidische Blicke auf sich ziehen.

- LEo für eine Überdosis an guter Laune, die er in unser Büro gebracht hat, in einer Zeit, in der es für mich im Labor nicht viel zu lachen gab.

- Liu, Daqing, Korenobu, Nir and Yazgan for being great office mates.

- Martin and Yannick for introducing me to this marvelous experiment.

- All remaining NANOs for being a wonderful group. You made me realize what Newton meant when he said: "If I have seen further it is by standing on the shoulders of Nanos ${ }^{1}$."

- Lothar, Adam und Oliver aus der Elektronikwerkstatt für die Reparatur und den Neubau vieler Geräte.

- Bernhard und Robert aus der Mechanikwerkstatt für die Anfertigung vieler kleiner und großer Werkstücke.

- Matthias und dem Rest DeR InfRastruktur für die Unterstützung in allen verwaltungstechnischen Belangen, durch die ich meine Konzentration ganz auf das Experiment richten konnte.

\footnotetext{
${ }^{1}$ Of course he said "giants" instead of "Nanos" - but only because he did not know you.
} 
This thesis examines the interaction of light with single dibenzanthanthrene (DBATT) dye molecules. DBATT serves as a model for a quantum-mechanical two-level system. By means of strong focusing of the incident light and cooling of the dye molecules to temperatures below $2 \mathrm{~K}$, a particularly efficient light-matter interaction can be realized. This enables observation of the nonlinearity inherent to a two-level system, e.g., in the form of saturation of the fluorescence signal with light beams containing only a few photons per lifetime of the excited molecular state.

Various nonlinear phenomena arise when two light beams of different frequency are sent to a single molecule. These processes can be exploited to coherently manipulate the transmission of a beam focused onto a single molecule via a second light beam. The occurrent effects, i.e., the AC-Stark shift, stimulated Rayleigh scattering, and three-photon amplification, are detected in the transmitted signal. In addition, four-wave mixing and the dependence of the excited state population on the phase difference of the two incident beams are demonstrated by the use of measurements with subnanosecond time resolution. These results show the possible application of organic dye molecules in the field of quantum information processing where nonlinearities on the single photon and single emitter level are highly sought-after.

In this work, the experimental and theoretical principles of single molecule spectroscopy are discussed. Particular attention is placed on the investigation of the coherent light-matter interaction using transmission measurements. A significant change in this transmitted signal, via the scattering of a single molecule, requires a strong light-matter coupling. To quantify the efficiency of this interaction, the maximum possible coupling of a focused beam and a single emitter is discussed. The results presented herein show that the achieved coupling is typically $5 \%$ of the theoretical maximum. Thus, the interaction of a molecule with two light fields with different frequencies is investigated. The nonlinear effects that arise are described qualitatively within the dressed-atom model and quantitatively with a Fourier ansatz. The experimental techniques are explained in detail and the measurement results are presented and discussed.

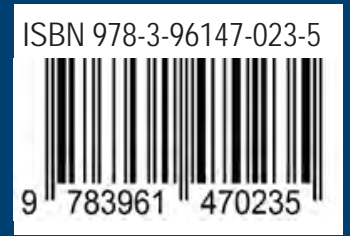

